\title{
Biomass equations for sixty-five North American tree species
}

\author{
Michael T. Ter-Mikaelian ${ }^{\mathrm{a},{ }^{*}}$, Michael D. Korzukhin ${ }^{\mathrm{b}}$ \\ a Ontario Forest Research Institute, 1235 Queen Street East. Sault Ste. Marie, Ont. P6A 2E5, Canada \\ ${ }^{b}$ Institute of Global Climate and Ecology, Moscow, 107258, Russia
}

Received 21 October 1996; accepted 6 January 1997

\begin{abstract}
The paper presents a comprehensive review of the biomass equations for 65 North American tree species. All equations are of the form $M=a D^{b}$, where $M$ is the oven-dry wcight of the biomass component of a tree $(\mathrm{kg}), D$ is diameter at breast height (DBH) (cm), and $a$ and $b$ are parameters. Equations for the following tree components were included in the review: total aboveground biomass, stem wood, stem bark, total stem (wood and bark), foliage, and branches (wood and bark). A total of 803 equations are presented with the range of DBH values of the sample, sample size, coefficient of determination $R^{2}$, standard error of the estimate, fitting method used to estimate the parameters $a$ and $b$, correction factor for a bias introduced by logarithmic transformation of the data, site index and geographic location of the sampled stand(s), and a reference to the paper in which the equation (or the data) was published. The review is a unique source of equations that can be used to estimate tree biomass and/or to study the variation of biomass components for a tree species. (1) 1997 Elsevier Science B.V.
\end{abstract}

Keywords: Aboveground biomass; Stem wood biomass; Stem bark biunass; Foliage biomass; Branch biomass; Dry weight

\section{Introduction}

In the last few decades, considerable research effort has gone into estimating the biomass of individual trees and relating it to tree characteristics such as diameter at breast height (DBH), total height, etc. Biomass equations for individual trees have been produced in studies of forest production and its correlation with stand density (Baskerville, 1965), in studies comparing biomass and production for individual tree species (Pastor and Bockheim, 1981), and in studies on forest fuel estimation (Agee, 1983), etc.

\footnotetext{
${ }^{*}$ Corresponding author. Tel.: (705) 946-2981; Fax: (705) 9462030 or (705) 946-2112; E-mail: termikmi@epo.gov.on.ca
}

As a result, several different biomass estimation equations are reported in the literature for the same species.

Whenever there is a need to estimate the biomass of individual trees, the abundance of existing predictive equations provides an alternative to destructive sampling of trees for the purposes of developing local equations. However, the user has to rely on estimates developed for other sites that are most likely different from the conditions on their particular site. Several approaches have been suggested to circumvent this problem: (a) find the geographically closest site; (b) use several reported equations to estimate the range of biomass (Tritton and Hornbeck, 1982); and (c) generate biomass data using various published equations and fit a new equation to the 
generated data (Pastor et al., 1984). Crow and Schlaegel (1988) provided a broad discussion of the application of biomass equations where several equations are available for a species.

Implementation of all three approaches, however, is hampered hecause the developed hiomass equations are scattered across a large body of forestry literature. Many equations are published in internal reports and are presented in conflicting formats that complicate their comparison. Several previous reviews of biomass equations either need to be updated (Stanek and State, 1978) or were designed to suit local geographic needs (Gholz et al., 1979; Tritton and Hornbeck, 1982).

The objective of this paper is to present a comprehensive and consistent review of biomass equations for North American tree species. The review can be used to estimate biomass or as a base to study the cross-site biomass variation of an individual tree species.

\section{Materials}

This review includes equations of the form:

$M=a D^{h}$

where $M$ is the oven-dry weight of the biomass component of a tree $(\mathrm{kg}), D$ is DBH $(\mathrm{cm})$, and $a$ and $b$ are parameters. Although the literature on individual-tree biomass estimation provides a number of equations that either have a form different from $\mathrm{Eq}$. (1) or that include additional independent variables such as tree height, sapwood area, etc., Eq. (1) (often presented in the logarithmic form) is most frequently reported.

The popularity of Eq. (1) in the literature stems from the fact that it provides a good balance of accurate predictions and low data requirements; using the most commonly and easily measured variable in forest studies (DBH). Addition of other tree variables, although statistically significant, does not usually lead to a substantial increase in $R^{2}$ or a decrease in SEE. For example, Freedman et al. (1982) noted that addition of total tree height (the second most common variable used to predict biomass) accounted for such a small variation in weight beyond that accounted for by DBH that "the chance of commit- ting an error by adding the height as a significant variable, when in fact it was not. was seldom less than 0.05 '. Similarly low gains from including height were demonstrated by Peterson et al. (1970), Crow (1971), Ralston (1973), Ker (1980a,b, 1984), Schmitt and Grigal (1981), Crow and Erdmann (1983). Hocker and Earley (1983), Ouellet (1983), Grigal and Kernik (1984b), Campbell et al. (1985), and Harding and Grigal (1985). Results from studies using other variables (e.g. sapwood area, Baldwin (1989), Bormann (1990); crown width and crown volume, Ker (1980a), etc.) were inconsistent. We therefore decided to omit equations other than $\mathrm{Eq}$. (1) from our review.

The review includes equations for: total aboveground biomass (AB), stem wood (SW), stem bark (SB), stem total (wood and bark) (ST), foliage (FL). and branches (wood and bark) (BR). Some authors have also reported separate equations for finer components. e.g. for previous year's or older - foliage (Bormann, 1990), but the components listed above are the most commonly reported.

First, we searched the literature to collect as many equations of the same type as Fq. (1) as possible. Original papers were reviewed to verify the study region, the measurement technique, the number of trees sampled, the range of values of the independent variable $(\mathrm{DBH})$, and the method used to fit the regression equation. Only equations fitted with data sampled in the original study were included in the revicw; we cxcluded "secondary" equations that were fitted with data generated from equations of a form other than that of Fq. (1). Two exceptions were made as follows.

(a) Perala and Alban (1994) reported two relationships for some species: height versus DBH and biomass component versus DBH and height. Since both relationships were fitted with data from the same sample, we substituted the first relationship for the height term in the second relationship.

(b) Although not clear from their text, we believe that to obtain a set of additive equations, Young et al. (1980) first fitted equations for biomass components, then calculated the sum of biomass components predicted by those equations for a set of incremental DBH values, and fitted the equation for total aboveground biomass to the predicted total aboveground biomass values. 
Finally, several authors reported original data used to fit equations different from Eq. (1). We used their data to fit equations of the form of Eq. (1); these equations are included in the review with reference to the original authors.

\section{Results}

Appendix A presents the list of equations for 65 North American tree species. The comments below explain some of the contents of Appendix A.

\subsection{Parameters $a$ and $b$}

All parameters refer to the form (1) of a corresponding biomass equation. Many authors have reported Eq. (1) in a logarithmic form, i.e. the parameters $\ln _{e} a$ (or $\log _{10} a$ ) and $b$ were estimated using a linear regression applied to the logarithm of biomass and $\mathrm{DBH}$ values. In these cases, we converted the parameters back to arithmetic units to make them comparable with those fitted using nonlinear regression. When necessary, parameters were converted to metric.

\subsection{DBH sample range ( $D$ range)}

An estimate is presented for several equations for which the authors did not provide a sample range for DBH. For equations by Wiant et al. (1977) and Young et al. (1980), the DBH sample range was estimated from the biomass tables presented in their papers; for equations by Whittaker et al. (1974) it was estimated from the DBH distribution of the stands sampled in their study. These estimates may, thus, exceed the actual sample range used for fitting the equations.

\subsection{Sample size $(N)$}

Wiant et al. (1977) reported a sample size between 19 and 22 for all the species included in their study; a conservative estimate of 19 is used in Appendix A.

\subsection{Coefficient of determination $\left(R^{2}\right)$}

When necessary, the $R^{2}$ values were calculated using the $R$ or adjusted $R^{2}$ values reported by the authors. It should be noted, that the $R^{2}$ values in Appendix $A$ are related to the regression method used to fit the parameters $a, b$ in the original study. The reader should therefore check the Fitting method (Mtd) column before comparing the $R^{2}$ values of equations for the same species.

\subsection{Standard error of estimate (SEE)}

Whittaker and Woodwell (1968), Whittaker et al. (1974), Koerper and Richardson (1980), and Pastor and Bockheim (1981) reported an error of estimate $E$ calculated as an antilog of the standard error of estimate SEE; in these cases, SEE was calculated as $\ln _{e}(E)$ or $\log _{10}(E)$ depending on the fitting method used in the paper. As with $R^{2}$, SEE is related to the regression method used to fit the original parameters, i.e. for equations fitted in the logarithmic form, SEE is given in corresponding logarithmic units.

\subsection{Correction factor (C.f.)}

Application of a linear regression to the log-transformed data introduces a systematic bias when the predicted values are converted back to arithmetic units. To compensate for this bias, Baskerville (1972) suggested using a correction factor calculated as an antilog of one half of the sample variance, the latter being equal to the SEE squared. To obtain an unbiased estimate, the predicted biomass values should be multiplied by this correction factor. For consistency, all parameters $a, b$ in the table specify raw (uncorrected) equations, including the equations from Gholz et al. (1979) and Snell and Little (1983) that are reported in a corrected form.

\subsection{Site index (SI)}

If provided in the source of equation, the site index is specified in Appendix A as the height (m) followed in brackets by the base age (years). The majority of the reviewed papers, however, had either none or little quantitative information about the sites sampled for tree biomass data. Denotations used for sites for which the authors provided a qualitative assessment are defined in Appendix A. It should be noted that for some species, Perala and Alban (1994) reported the basic equations and correction factors 
for specific sites; for these species, only parameters for the basic equation are included in Appendix A.

\section{Acknowledgements}

This publication was supported by VMAP (Vegetation Management Alternatives Program) through the Ontario Ministry of Natural Resources, Sault Stc. Marie, Ontario. We thank Lisa Buse for editing an earlier draft of this manuscript and two anonymous reviewers from the joumal for helpful comments.

\section{Appendix A. Individual tree biomass equations of the form $M=a D^{b}$, where $D$ is the diameter at breast height $(\mathrm{cm})$, and $M$ is the aboveground biomass component ( $\mathrm{kg}$ of oven-dried weight)}

For each equation, the table includes parameters $a$ and $b$, DBH sample range ( $D$ range) $(\mathrm{cm})$, the sample size $(N)$, coefficient of determination $\left(R^{2}\right)$, standard error of estimate (SEE), fitting method (Mtd) used to estimate parameters $a$ and $b$, correction factor (C.f.) for a bias introduced by logarithmic transformation of the data, site index (SI) of the sampled stand(s) specified as the height $(\mathrm{m})$ followed in brackets by the base age (years), geographic location (Region) of the sampled stand(s). and a reference to the paper (Author) in which the equation (or the data) was published: If missing, the corresponding column indicates $n / a$ (not available). The following denotations are used.

1. Biomass components $(\mathrm{M})$ : $\mathrm{AB}$ for total aboveground biomass; SW for stem wood; SB for stem bark; ST for total stem biomass (wood + bark); FL for foliage biomass; BR for total biomass of branches (wood + bark). Where the first column is blank, the line refers to the last specified biomass component.

2. Fitting method (Mtd): abs or absw for equations fitted with a nonlinear or weighted nonlinear regression, respectively; In or log for equations fitted using linear regression applied to $\log _{e^{-}}$or $\log _{10}$ transformed data, respectively; calc for equations calculated from two or more equations (see Section 2).

3. Site index (SI): comp for the data from various sites pooled together; intermed for intermicdiate sites, good and poor for good and poor sites, respectively, with a prefix, $c$, if the data were pooled from several good or several poor sites.

\begin{tabular}{|c|c|c|c|c|c|c|c|c|c|c|c|}
\hline$M$ & $a$ & $b$ & $D$ range & $N$ & $R^{2}$ & SEE & Mtd & C.f. & SI & Region & Author \\
\hline \multicolumn{12}{|c|}{ Alder, red ( Alnus rubra Bong.) } \\
\hline FL: & 0.0100 & 1.9398 & $3-63$ & 53 & 0.929 & 0.444 & $\ln$ & 1.104 & comp & Oregon, Washington & Snell and Little, 198 \\
\hline BR: & 0.0069 & 2.6516 & $3-63$ & 53 & 0.936 & 0.574 & $\ln$ & 1.179 & comp & Oregon, Washington & Snell and Little, 198 \\
\hline \multicolumn{12}{|c|}{ Alder, speckled ( Alnus rugosa (DuRoi) Spreng.) } \\
\hline $\mathrm{AB}:$ & 0.2612 & 2.2087 & $3-9^{a}$ & 30 & $\mathrm{n} / \mathrm{a}$ & $n / a$ & calc & $\mathrm{n} / \mathrm{a}$ & comp & Maine & Young et al.. 1980 \\
\hline \multirow[t]{2}{*}{ ST: } & 0.0456 & 2.5847 & $3-8$ & 30 & 0.934 & $n / a$ & $\log$ & $n / a$ & comp & Maine & Ribe, 1973 \\
\hline & 0.0463 & 2.5755 & $3-9^{ \pm}$ & 30 & 0.967 & $n / a$ & $\ln$ & $n / a$ & comp & Maine & Young et al., 1980 \\
\hline \multirow[t]{2}{*}{ FL: } & 0.0461 & 1.2643 & $3-8$ & 30 & 0.667 & $\mathrm{n} / \mathrm{a}$ & $\log$ & $\mathrm{n} / \mathrm{a}$ & comp & Maine & Ribe. 1973 \\
\hline & 0.0479 & 1.2274 & $3-9$ & 30 & 0.802 & $\mathrm{n} / \mathrm{a}$ & $\ln$ & $\mathrm{n} / \mathrm{a}$ & comp & Maine & Young et al.. 1980 \\
\hline \multirow[t]{2}{*}{ BR: } & 0.0620 & 1.5184 & $3-8$ & 30 & 0.776 & $\mathrm{n} / \mathrm{a}$ & $\log$ & $\mathrm{n} / \mathrm{a}$ & comp & Maine & Ribe, 1973 \\
\hline & 0.0617 & 1.5201 & $3-9$ & 30 & 0.879 & $\mathrm{n} / \mathrm{a}$ & $\ln$ & $\mathrm{n} / \mathrm{a}$ & comp & Maine & Young et al. 1180 \\
\hline
\end{tabular}

Ash, black (Fraxinus nigra Marsh.)

\begin{tabular}{|c|c|c|c|c|c|c|c|c|c|c|c|}
\hline AB: & 0.1634 & 2.3480 & $4-32$ & 18 & $n / a$ & $\mathrm{n} / \mathrm{a}$ & calc & $\mathrm{n} / \mathrm{a}$ & $\mathrm{n} / \mathrm{a}$ & Upper Great Lakes & Peraia and Alban, 1994 \\
\hline SW: & 0.0926 & 2.3879 & $4-32$ & 17 & $\mathrm{n} / \mathrm{a}$ & $\mathrm{n} / \mathrm{a}$ & calc & $\mathrm{n} / \mathrm{a}$ & $\mathrm{n} / \mathrm{a}$ & Upper Great Lakes & Perala and Alban, 1994 \\
\hline SB: & 0.0275 & 2.1002 & $4-32$ & 17 & $\mathrm{n} / \mathrm{a}$ & $\mathrm{n} / \mathrm{a}$ & calc & $\mathrm{n} / \mathrm{a}$ & $n / a$ & Upper Great Lakes & Perala and Alban, 1994 \\
\hline FL: & 0.0026 & 2.4160 & $4-32$ & 17 & 0.953 & 0.348 & $\ln$ & 1.062 & $\mathrm{n} / \mathrm{a}$ & Upper Great Lakes & Perala and Alban, 1994 \\
\hline \multicolumn{12}{|c|}{ Ash, white (Fraxinus americana $\mathrm{I}$.) } \\
\hline \multirow[t]{3}{*}{$\mathrm{AB}:$} & 0.1063 & 2.4798 & $5-50$ & 15 & 0.990 & $\mathrm{n} / \mathrm{a}$ & & $\mathrm{n} / \mathrm{at}$ & $\mathrm{n} / \mathrm{a}$ & West Virginia & Brenneman et al., 1978 \\
\hline & 0.1535 & 2.3213 & $1-28$ & 46 & 0.992 & 0.141 & $\ln$ & 1.010 & $\mathrm{n} / \mathrm{a}$ & New Brunswick & Ker, $1980 \mathrm{a}$ \\
\hline & 0.1634 & 2.3480 & $4-32$ & 18 & $n / a$ & $\mathrm{n} / \mathrm{a}$ & calc & $n / a$ & $n / a$ & Upper Great Lakes & Perala and Alban, 1994 \\
\hline \multirow[t]{2}{*}{ SW: } & 0.0936 & 2.3903 & $1-28$ & 47 & 0.992 & 0.141 & $\ln$ & 1.010 & $\mathrm{n} / \mathrm{a}$ & New Brunswick & Ker, $1980 \mathrm{a}$ \\
\hline & 0.0926 & 2.3879 & $4-32$ & 17 & $\mathrm{n} / \mathrm{a}$ & $\mathrm{n} / \mathrm{a}$ & calc & $\mathrm{n} / \mathrm{a}$ & $\mathrm{n} / \mathrm{a}$ & Upper Great Lakes & Perala and Alban, 1994 \\
\hline
\end{tabular}




$\begin{array}{llllrllllll}\text { SB: } & 0.0198 & 2.1762 & 1-28 & 47 & 0.971 & 0.243 & \ln & 1.030 & \text { n/a } & \text { New Brunswick } \\ & 0.0275 & 2.1002 & 4-32 & 17 & \mathrm{n} / \mathrm{a} & \mathrm{n} / \mathrm{a} & \text { calc } & \mathrm{n} / \mathrm{a} & \mathrm{n} / \mathrm{a} & \text { Upper Great Lakes } \\ \text { ST: } & 0.0909 & 2.5600 & 1-21 & 14 & 0.996 & 0.205 & \ln & 1.021 & \text { comp } & \text { New Hampshire } \\ & 0.1124 & 2.3649 & 1-28 & 47 & 0.991 & 0.141 & \ln & 1.010 & \mathrm{n} / \mathrm{a} & \text { New Brunswick } \\ \text { FL: } & 0.0182 & 1.7000 & 1-21 & 8 & 0.839 & 0.778 & \ln & 1.353 & \text { comp } & \text { New Hampshire } \\ & 0.0163 & 1.6932 & 1-28 & 46 & 0.935 & 0.280 & \mathrm{ln} & 1.040 & \mathrm{n} / \mathrm{a} & \text { New Brunswick } \\ & 0.0026 & 2.4160 & 4-32 & 17 & 0.953 & 0.348 & \mathrm{ln} & 1.062 & \mathrm{n} / \mathrm{a} & \text { Upper Great Lakes } \\ \text { BR: } & 0.0123 & 2.5400 & 1-21 & 14 & 0.973 & 0.507 & \ln & 1.137 & \text { comp } & \text { New Hampshire } \\ & 0.0315 & 2.1935 & 1-28 & 46 & 0.927 & 0.312 & \mathrm{ln} & 1.050 & \mathrm{n} / \mathrm{a} & \text { New Brunswick }\end{array}$

Aspen, largetooth (Populus grandidentata Michx.)

\begin{tabular}{|c|c|c|c|c|c|c|c|c|c|c|}
\hline \multirow[t]{2}{*}{$\mathrm{AB}$} & 0.0983 & 2.3773 & $1-34$ & 30 & 0.995 & 0.156 & $\ln$ & 1.012 & $\mathrm{n} / \mathrm{a}$ & Nova Scotia \\
\hline & 0.0785 & 2.4981 & $3-45$ & 57 & $\mathrm{n} / \mathrm{a}$ & $n / a$ & calc & $n / a$ & $17(50)$ & Upper Great Lakes \\
\hline \multirow[t]{6}{*}{ SW: } & 0.0128 & 2.8586 & $1-34$ & 23 & 0.966 & 0.225 & in & 1.026 & $n / a$ & Nova Scotia \\
\hline & 0.0362 & 2.6544 & $\mathrm{n} / \mathrm{a}$ & 31 & 0.980 & 0.191 & $\ln$ & 1.018 & comp & Michigan \\
\hline & 0.1059 & 2.3488 & $n / a$ & 10 & 0.990 & 0.095 & $\ln$ & 1.005 & good & Michigan \\
\hline & 0.0503 & 2.5478 & $\mathrm{n} / \mathrm{a}$ & 11 & 0.980 & 0.174 & $\ln$ & 1.015 & $\begin{array}{l}\text { inter- } \\
\text { med }\end{array}$ & Michigan \\
\hline & 0.0467 & 2.4932 & $n / a$ & 10 & 0.980 & 0.166 & $\ln$ & 1.014 & poor & Michigan \\
\hline & 0.0426 & 2.5618 & $3-45$ & 58 & $n / a$ & $\mathrm{n} / \mathrm{a}$ & calc & $\mathrm{n} / \mathrm{a}$ & $17(50)$ & Upper Great Lakes \\
\hline \multirow[t]{6}{*}{ SB: } & 0.0076 & 2.6158 & $1-34$ & 23 & 0.958 & 0.231 & In & 1.027 & $n / a$ & Nova Scotia \\
\hline & 0.0307 & 2.2034 & $\mathrm{n} / \mathrm{a}$ & 31 & 0.970 & 0.182 & ln & 1.017 & comp & Michigan \\
\hline & 0.0541 & 2.0610 & $\mathrm{n} / \mathrm{a}$ & 10 & 0.990 & 0.068 & In & 1.002 & good & Michigan \\
\hline & 0.0419 & 2.1047 & $\mathrm{n} / \mathrm{a}$ & 11 & 0.980 & 0.140 & $\ln$ & 1.010 & $\begin{array}{l}\text { inter- } \\
\text { med }\end{array}$ & Michigan \\
\hline & 0.0488 & 1.9584 & $\mathrm{n} / \mathrm{a}$ & 10 & 0.980 & 0.148 & $\ln$ & 1.011 & poor & Michigan \\
\hline & 0.0333 & 2.2142 & $3-45$ & 56 & $\mathrm{n} / \mathrm{a}$ & $\mathrm{n} / \mathrm{a}$ & calc & $\mathrm{n} / \mathrm{a}$ & $17(50)$ & Upper Great Lakes \\
\hline ST: & 0.0192 & 2.8093 & $1-34$ & 23 & 0.969 & 0.212 & In & 1.023 & $n / a$ & Nova Scotia \\
\hline \multirow[t]{6}{*}{ FL: } & 0.0159 & 1.7369 & $1-34$ & 30 & 0.951 & 0.376 & $\ln$ & 1.073 & $\mathrm{n} / \mathrm{a}$ & Nova Scotia \\
\hline & 0.0036 & 2.1483 & $\mathrm{n} / \mathrm{a}$ & 31 & 0.850 & 0.438 & $\ln$ & 1.101 & comp & Michigan \\
\hline & 0.0001 & 3.2307 & $n / a$ & 10 & 0.880 & 0.392 & $\ln$ & 1.080 & good & Michigan \\
\hline & 0.0009 & 2.6130 & $\mathrm{n} / \mathrm{a}$ & 11 & 0.920 & 0.365 & $\ln$ & 1.069 & $\begin{array}{l}\text { inter- } \\
\text { med }\end{array}$ & Michigan \\
\hline & 0.0082 & 1.9236 & $\mathrm{n} / \mathrm{a}$ & 10 & 0.940 & 0.247 & $\ln$ & 1.031 & poor & Michigan \\
\hline & 0.0027 & 2.2750 & $3-45$ & 57 & 0.852 & 0.487 & $\ln$ & 1.126 & $17(50)$ & Upper Great Lakes \\
\hline $3 R$ & 0.1368 & 1.7510 & $1-34$ & 30 & 0.963 & 0.327 & In & 1.055 & $\mathrm{n} / \mathrm{a}$ & Nova Scotia \\
\hline
\end{tabular}

Aspen, trembling (Populus tremuloides Michx.)

\begin{tabular}{|c|c|c|c|c|c|c|c|c|c|c|}
\hline \multirow[t]{8}{*}{$\mathrm{AB}:$} & 0.1008 & 2.4341 & $1-30$ & 21 & 0.998 & 0.100 & $\ln$ & 1.005 & comp & Alberta \\
\hline & 0.0790 & 2.3865 & $1-32$ & 23 & 0.995 & 0.120 & $\ln$ & 1.007 & comp & Alberta \\
\hline & 0.0911 & 2.2759 & $1-26$ & 22 & 0.987 & 0.173 & $\ln$ & 1.015 & comp & Alberta \\
\hline & 0.1122 & 2.3500 & $1-32$ & 34 & 0.983 & 0.270 & $\ln$ & 1.037 & comp & Yukon \\
\hline & 0.0928 & 2.4085 & $1-27$ & 26 & 0.995 & 0.162 & $\ln$ & 1.013 & $\mathrm{n} / \mathrm{a}$ & Nova Scotia \\
\hline & 0.1231 & 2.2420 & $3-36$ & 20 & 0.971 & 0.300 & $\ln$ & 1.046 & comp & Utah, Wyoming \\
\hline & 0.0726 & 2.4827 & $2-33$ & 46 & 0.990 & 0.141 & $\ln$ & 1.010 & $\mathrm{n} / \mathrm{a}$ & Nova Scotia \\
\hline & 0.1049 & 2.3910 & $0-36$ & 197 & 0.989 & 19.50 & abs & $\mathrm{n} / \mathrm{a}$ & comp & $\begin{array}{l}\text { New Brunswick, } \\
\text { Nova Scotia }\end{array}$ \\
\hline \multirow{10}{*}{ SW: } & 0.1625 & 2.0673 & $0-15$ & 15 & 0.951 & $\mathrm{n} / \mathrm{a}$ & $\log$ & $\mathrm{n} / \mathrm{a}$ & $\mathrm{n} / \mathrm{a}$ & New Brunswick \\
\hline & 0.2065 & 2.2490 & $15-40$ & 9 & 0.988 & 0.037 & $\log$ & 1.002 & $21.5(50)$ & Wisconsin \\
\hline & 0.0527 & 2.5084 & $3-50$ & 118 & $\mathrm{n} / \mathrm{a}$ & $\mathrm{n} / \mathrm{a}$ & calc & $\mathrm{n} / \mathrm{a}$ & $17(50)$ & Upper Great Lakes \\
\hline & 0.0774 & 2.3466 & $5-33$ & 49 & 0.958 & 0.099 & $\log$ & 1.011 & $\mathrm{n} / \mathrm{a}$ & Alberta \\
\hline & 0.0637 & 2.6087 & $3-51^{a}$ & 52 & $\mathrm{n} / \mathrm{a}$ & $\mathrm{n} / \mathrm{a}$ & calc & $\mathrm{n} / \mathrm{a}$ & comp & Maine \\
\hline & 0.0332 & 2.5816 & $1-27$ & 16 & 0.939 & 0.168 & $\ln$ & 1.014 & $\mathrm{n} / \mathrm{a}$ & Nova Scotia \\
\hline & 0.0675 & 2.2450 & $3-36$ & 20 & 0.966 & 0.318 & $\ln$ & 1.052 & comp & Utah, Wyoming \\
\hline & 0.0419 & 2.5325 & $2-33$ & 46 & 0.990 & 0.141 & $\ln$ & 1.010 & $n / a$ & Nova Scotia \\
\hline & 0.0639 & 2.3938 & $0-36$ & 197 & 0.986 & 13.60 & abs & $\mathrm{n} / \mathrm{a}$ & comp & $\begin{array}{l}\text { New Brunswick, } \\
\text { Nova Scotia }\end{array}$ \\
\hline & 0.1714 & 2.1990 & $15-40$ & 9 & 0.986 & 0.041 & $\log$ & 1.002 & $21.5(50)$ & Wisconsin \\
\hline
\end{tabular}

Ker, 1980a

Perala and Alban, 1994

Hocker and Earley, 1983

Ker, 1980a

Hocker and Earley, 1983

Ker, 1980a

Perala and Alban, 1994

Hocker and Earley, 1983

Ker, 1980a
Freedman et al., 1982

Perala and Alban, 1994

Freedman et al., 1982

Koerper and Richardson, 1980

Koerper and Richardson, 1980

Koerper and Richardson, 1980

Koerper and Richardson, 1980

Perala and Alban. 1994

Freedman et al., 1982

Koerper and Richardson, 1980

Koerper and Richardson, 1980

Koerper and Richardson, 1980

Koerper and Richardson, 1980

Perala and Alban, 1994

Freedman et al., 1982

Freedman et al., 1982

Koerper and Richardson, 1980

Koerper and Richardson, 1980

Koerper and Richardson, 1980

Koerper and Richardson, 1980

Perala and Alban, 1994

Freedman et al., 1982

Campbell et al., 1985

Campbell et al., 1985

Campbell et al., 1985

Campbell et al., 1985

Freedman et al., 1982

Johnston and Bartos, $1977^{\mathrm{b}}$

Ker, $1980 b$

Ker, 1984

MacLean and Wein, 1976

Pastor and Bockheim, 1981

Perala and Alban, 1994

Peterson et al., 1970

Young et al., 1980

Freedman et al., 1982

Johnston and Bartos, $1977^{\mathrm{b}}$

Ker, 1980 b

Ker, 1984

Pastor and Bockheim, 1981 


\begin{tabular}{|c|c|c|c|c|c|c|c|c|c|c|}
\hline & 0.0326 & 2.5178 & $3-50$ & 119 & $\mathrm{n} / \mathrm{a}$ & $n / a$ & calc & $\mathrm{n} / \mathrm{a}$ & $17(50)$ & Upper Great Lakes \\
\hline & 0.0407 & 2.6060 & $3-31$ & 45 & 0.993 & 0.141 & $\ln$ & 1.010 & comp & Wisconsin \\
\hline \multirow[t]{6}{*}{ SB: } & 0.0199 & 2.3528 & $1-27$ & 16 & 0.933 & 0.162 & $\ln$ & 1.013 & $\mathrm{n} / \mathrm{a}$ & Nova Scotia \\
\hline & 0.0339 & 2.1440 & $3-36$ & 20 & 0.956 & 0.351 & $\ln$ & 1.064 & comp & Utah, Wyoming \\
\hline & 0.0139 & 2.4007 & $2-33$ & 46 & 0.980 & 0.243 & $\ln$ & 1.030 & $\mathrm{n} / \mathrm{a}$ & Nova Scotia \\
\hline & 0.0437 & 2.1460 & $15-40$ & 9 & 0.955 & 0.076 & $\log$ & 1.007 & $21.5(50)$ & Wisconsin \\
\hline & 0.0113 & 2.3198 & $3-50$ & 120 & $\mathrm{n} / \mathrm{a}$ & $\mathrm{n} / \mathrm{a}$ & calc & $\mathrm{n} / \mathrm{a}$ & $17(50)$ & Upper Great Lakes \\
\hline & 0.0108 & 2.5520 & $3-31$ & 45 & 0.981 & 0.235 & $\ln$ & 1.028 & comp & Wisconsin \\
\hline \multirow[t]{9}{*}{ ST: } & 0.0508 & 2.5293 & $1-27$ & 16 & 0.951 & 0.147 & $\ln$ & 1.011 & $\mathrm{n} / \mathrm{a}$ & Nova Scotiá \\
\hline & 0.0985 & 2.1300 & $0-15$ & 79 & 0.980 & 0.211 & $\ln$ & 1.023 & comp & New Hampshire \\
\hline & 0.1007 & 2.2190 & $3-36$ & 20 & 0.968 & 0.307 & $\ln$ & 1.048 & comp & Utah, Wyoming \\
\hline & 0.0558 & 2.5046 & $2-33$ & 46 & 0.990 & 0.141 & $\ln$ & 1.010 & $n / a$ & Nova Scotia \\
\hline & 0.0774 & 2.3971 & $0-36$ & 197 & 0.986 & 16.80 & abs & $n / a$ & comp & $\begin{array}{l}\text { New Brunswick, } \\
\text { Nova Scotia }\end{array}$ \\
\hline & 0.0985 & 2.1426 & $0-15$ & 15 & 0.956 & $n / a$ & $\log$ & $\mathrm{n} / \mathrm{a}$ & $n / a$ & New Brunswick \\
\hline & 0.0647 & 2.3564 & $5-33$ & 49 & 0.947 & 0.112 & $\log$ & 1.015 & $\mathrm{n} / \mathrm{a}$ & Alberta \\
\hline & 0.0346 & 2.7859 & $3-15$ & 30 & 0.990 & $n / a$ & $\log$ & $\mathrm{n} / \mathrm{a}$ & comp & Maine \\
\hline & 0.0448 & 2.6709 & $3-51^{a}$ & 52 & 0.995 & $\mathrm{n} / \mathrm{a}$ & $\ln$ & $\mathrm{n} / \mathrm{a}$ & comp & Maine \\
\hline \multirow[t]{11}{*}{ FL: } & 0.0177 & 1.6093 & $1-27$ & 26 & 0.859 & 0.615 & In & 1.208 & $\mathrm{n} / \mathrm{a}$ & Nova Scotia \\
\hline & 0.0191 & 2.0900 & $0-15$ & 22 & 0.980 & 0.211 & $\ln$ & 1.023 & comp & New Hampshire \\
\hline & 0.0192 & 1.5470 & $3-36$ & 20 & 0.841 & 0.524 & In & 1.147 & comp & Utah, Wyoming \\
\hline & 0.0099 & 1.8405 & $2-33$ & 46 & 0.930 & 0.341 & In & 1.060 & $n / a$ & Nova Scotia \\
\hline & 0.0198 & 1.8031 & $0-36$ & 197 & 0.874 & 1.900 & abs & $\mathrm{n} / \mathrm{a}$ & comp & $\begin{array}{l}\text { New Brunswick. } \\
\text { Nova Scotia }\end{array}$ \\
\hline & 0.0243 & 1.4920 & $15-40$ & 9 & 0.757 & 0.134 & $\log$ & 1.021 & $21.5(50)$ & Wisconsin \\
\hline & 0.0114 & 2.0261 & $3-50$ & 102 & $\mathrm{n} / \mathrm{a}$ & $\mathrm{n} / \mathrm{a}$ & calc & $\mathrm{n} / \mathrm{a}$ & $17(50)$ & Upper Great Lakes \\
\hline & 0.0050 & 1.9742 & $5-33$ & 49 & 0.931 & 0.109 & $\log$ & 1.014 & $n / a$ & Alberta \\
\hline & 0.0221 & 1.6796 & $3-15$ & 30 & 0.750 & $n / a$ & $\log$ & $\mathrm{n} / \mathrm{a}$ & comp & Maine \\
\hline & 0.0130 & 1.8680 & $3-31$ & 45 & 0.967 & 0.231 & $\ln$ & 1.027 & comp & Wisconsin \\
\hline & 0.0110 & 2.0766 & $3-51^{a}$ & 52 & 0.949 & $\mathrm{n} / \mathrm{a}$ & $\ln$ & $n / a$ & comp & Maine \\
\hline \multirow[t]{10}{*}{ BR: } & 0.1684 & 1.6262 & $1-27$ & 26 & 0.903 & 0.502 & $\ln$ & 1.134 & $\mathrm{n} / \mathrm{a}$ & Nova Scotia \\
\hline & 0.0958 & 2.7200 & $0-15$ & 79 & 0.942 & 0.466 & In & 1.115 & comp & New Hampshire \\
\hline & 0.0112 & 2.4950 & $3-36$ & 20 & 0.935 & 0.512 & $\ln$ & 1.140 & comp & Utah, Wyoming \\
\hline & 0.0073 & 2.5995 & $2-33$ & 46 & 0.950 & 0.415 & $\ln$ & 1.090 & $\mathrm{n} / \mathrm{d}$ & Nova Scotia \\
\hline & 0.0192 & 2.4468 & $0-36$ & 197 & 0.872 & 15.80 & $a b s$ & $\mathrm{n} / \mathrm{a}$ & comp & $\begin{array}{l}\text { New Brunswick, } \\
\text { Nova Scotia }\end{array}$ \\
\hline & 0.0038 & 2.7680 & $15-40$ & 9 & 0.897 & 0.149 & $\log$ & 1.026 & $21.5(50)$ & Wisconsin \\
\hline & 0.0080 & 2.3708 & $5-33$ & 49 & 0.931 & 0.129 & $\log$ & 1.019 & $\mathrm{n} / \mathrm{a}$ & Alberta \\
\hline & 0.0293 & 1.8545 & $3-15$ & 30 & 0.853 & $\mathrm{n} / \mathrm{a}$ & $\log$ & $\mathrm{n} / \mathrm{a}$ & comp & Maine \\
\hline & 0.0065 & 2.6950 & $3-31$ & 45 & 0.982 & 0.247 & $\ln$ & 1.031 & comp & Wisconsin \\
\hline & 0.0082 & 2.5244 & $3-51^{i+}$ & 52 & 0.958 & $\mathrm{n} / \mathrm{a}$ & $\ln$ & $n / a$ & comp & Maine \\
\hline
\end{tabular}

Basswood (Tilia americana L.)

$\begin{array}{llll}\text { AB: } & 0.0617 & 2.5328 & 5-50 \\ & 0.0872 & 2.3539 & 4-47 \\ \text { SW: } & 0.0499 & 2.4024 & 4-47 \\ \text { SB: } & 0.0432 & 2.0339 & 4-47 \\ \text { ST: } & 0.0730 & 2.2900 & 2-10 \\ \text { FL: } & 0.0465 & 0.7100 & 2-10 \\ & 0.0049 & 2.0940 & 4-47 \\ \text { BR: } & 0.0389 & 1.8400 & 2-10\end{array}$

$\begin{array}{rllll}13 & 0.960 & \mathrm{n} / \mathrm{a} & \log & \mathrm{n} / \mathrm{a} \\ 31 & \mathrm{n} / \mathrm{a} & \mathrm{n} / \mathrm{a} & \mathrm{calc} & \mathrm{n} / \mathrm{a} \\ 31 & \mathrm{n} / \mathrm{a} & \mathrm{n} / \mathrm{a} & \mathrm{calc} & \mathrm{n} / \mathrm{a} \\ 31 & \mathrm{n} / \mathrm{a} & \mathrm{n} / \mathrm{a} & \mathrm{calc} & \mathrm{n} / \mathrm{a} \\ 5 & 0.984 & 0.254 & \ln & 1.033 \\ 5 & 0.818 & 0.300 & \ln & 1.046 \\ 30 & 0.874 & 0.511 & \ln & 1.139 \\ 5 & 0.911 & 0.51 \mathrm{l} & \ln & 1.139\end{array}$

$n / a$

$n / a$

$\mathrm{n} / \mathrm{a}$

$\mathrm{n} / \mathrm{a}$

comp

comp

$\mathrm{n} / \mathrm{a}$

comp
West Virginid

Upper Great Lakes

Upper Great Lakes

Upper Great Lakes

New Hampshire

New Hampshire

Upper Great Lakes

New Hampshire
Perala and Alban, 1994

Ruark ct al., 1987

Freedman et at.. 1982

Johnston and Bartos, $1977^{\text {b }}$

Ker, 1980b

Pastor and Bockheim, 1981

Perala and Alban. 1994

Ruark et al.. 1987

Freedman et al.. 1982

Hocker and Earley, 1983

Johnston and Bartos, $1977^{\text {b }}$

Ker, $1980 \mathrm{~b}$

Ker, 1984

MacLean and Wein. 1976

Peterson et al. 1970

Ribe. 1973

Young ef al., 1980

Freedman et al. 1982

Hocker and Earley, 1983

Johnston and Bartos, $1977^{\circ}$

Ker, 1980b

Ker, 1984

Pastor and Bockheim, 1981

Perala and Alban, 1994

Peterson et al., 1970

Ribe, 1973

Ruark et al., 1987

Young et al., 1980)

Freedman et al.. 1982

Hocker and Earley, 1983

Johnston and Bartos, $1977^{\mathrm{h}}$

Ker. 1980 b

Ker, 1984

Pastor and Bockheim, 1981

Peterson et al. 1970

Ribe, 1973

Ruark et al.. 1987

Young et al. 1980

Brenneman et al., 1978

Perala and Alban, 1994

Perala and Alban, 1994

Perala and Alban, 1994

Hocker and Earley, 1983

Hocker and Earley, 1983

Perala and Alban, 1994

Hocker and Earley, 1983

Beech, American (Fagus grandifolia Ehrh.)

$\begin{array}{lllllllllll}\text { AB: } & 0.0842 & 2.5715 & 5-50 & 56 & 0.970 & \mathrm{n} / \mathrm{a} & \log & \mathrm{n} / \mathrm{a} & \mathrm{n} / \mathrm{a} & \text { West Virginia } \\ 0.1958 & 2.2538 & 2-29 & 46 & 0.988 & 0.141 & \ln & 1.010 & \mathrm{n} / \mathrm{a} & \text { New Brunswick } \\ 0.1957 & 2.3916 & 1-60^{\mathrm{a}} & 14 & 0.994 & 0.089 & \operatorname{lng} & 1.009 & \mathrm{n} / \mathrm{a} & \text { New Hampshire } \\ 0.2013 & 2.2988 & 3-66^{\mathrm{a}} & 29 & \mathrm{n} / \mathrm{a} & \mathrm{n} / \mathrm{a} & \mathrm{calc} & \mathrm{n} / \mathrm{a} & \mathrm{comp} & \text { Maine }\end{array}$

Brenneman et ạl.. 1978

Ker, 1980a

Whittaker et al., 1974

Young et al., 1980 


$\begin{array}{lllllllllll}\text { SW: } & 0.1229 & 2.2956 & 2-29 & 47 & 0.987 & 0.141 & \ln & 1.010 & \mathrm{n} / \mathrm{a} & \text { New Brunswick } \\ & 0.0959 & 2.4113 & 1-60^{\mathrm{a}} & 14 & 0.996 & 0.080 & \log & 1.007 & \mathrm{n} / \mathrm{a} & \text { New Hampshirc } \\ \mathrm{SB}: & 0.0155 & 2.1154 & 2-29 & 47 & 0.979 & 0.243 & \ln & 1.030 & \mathrm{n} / \mathrm{a} & \text { New Brunswick } \\ & 0.0107 & 2.2450 & 1-60^{\mathrm{a}} & 14 & 0.994 & 0.090 & \log & 1.009 & \mathrm{n} / \mathrm{a} & \text { New Hampshire } \\ \mathrm{ST}: & 0.0937 & 2.4700 & 1-42 & 19 & 0.996 & 0.178 & \ln & 1.016 & \mathrm{comp} & \text { New Hampshire } \\ & 0.1381 & 2.2809 & 2-29 & 47 & 0.988 & 0.141 & \ln & 1.010 & \mathrm{n} / \mathrm{a} & \text { New Brunswick } \\ & 0.1155 & 2.4868 & 3-15 & 19 & 0.987 & \mathrm{n} / \mathrm{a} & \log & \mathrm{n} / \mathrm{a} & \text { comp } & \text { Maine } \\ & 0.1067 & 2.3981 & 1-60^{\mathrm{a}} & 14 & 0.996 & 0.079 & \log & 1.007 & \mathrm{n} / \mathrm{a} & \text { New Hampshire } \\ & 0.1515 & 2.2997 & 3-66^{\mathrm{a}} & 29 & 0.991 & \mathrm{n} / \mathrm{a} & \ln & \mathrm{n} / \mathrm{a} & \text { comp } & \text { Maine } \\ \text { FL: } & 0.0250 & 1.8300 & 1-42 & 12 & 0.979 & 0.357 & \ln & 1.066 & \text { comp } & \text { New Hampshire } \\ & 0.0233 & 1.6303 & 2-29 & 47 & 0.869 & 0.341 & \ln & 1.060 & \mathrm{n} / \mathrm{a} & \text { New Brunswick } \\ & 0.0216 & 1.8089 & 3-15 & 19 & 0.853 & \mathrm{n} / \mathrm{a} & \log & \mathrm{n} / \mathrm{a} & \mathrm{comp} & \text { Maine } \\ & 0.0183 & 1.9158 & 3-66^{\mathrm{a}} & 29 & 0.940 & \mathrm{n} / \mathrm{a} & \ln & \mathrm{n} / \mathrm{a} & \text { comp } & \text { Maine } \\ \text { BR: } & 0.0421 & 2.4100 & 1-42 & 19 & 0.981 & 0.300 & \ln & 1.046 & \text { comp } & \text { New Hampshire } \\ & 0.0274 & 2.3708 & 2-29 & 47 & 0.892 & 0.494 & \ln & 1.130 & \mathrm{n} / \mathrm{a} & \text { New Brunswick } \\ & 0.0944 & 1.5402 & 3-15 & 19 & 0.791 & \mathrm{n} / \mathrm{a} & \log & \mathrm{n} / \mathrm{a} & \text { comp } & \text { Maine } \\ & 0.0262 & 2.5509 & 1-60^{a} & 14 & 0.980 & 0.180 & \log & 1.038 & \mathrm{n} / \mathrm{a} & \text { New Hampshire } \\ & 0.0265 & 2.3634 & 3-66^{\mathrm{a}} & 29 & 0.931 & \mathrm{n} / \mathrm{a} & \ln & \mathrm{n} / \mathrm{a} & \mathrm{comp} & \text { Maine }\end{array}$

Birch, black ( Betula lenta L.)

$\mathrm{AB}: \quad 0.0629 \quad 2.6606 \quad 5-50$

ST: $\quad 0.0946 \quad 2.4900 \quad 2-10$

FL: $\quad 0.0045 \quad 2.4200 \quad 2-10$

BR: $0.0036 \quad 3.4200 \quad 2-10$

$\begin{array}{lllll}8 & 0.990 & \text { n } / a & \log & \text { II } / \mathrm{a} \\ 5 & 0.992 & 0.197 & \ln & 1.020 \\ 5 & 0.936 & 0.545 & \ln & 1.160 \\ 5 & 0.982 & 0.400 & \text { ln } & 1.083\end{array}$

$\mathrm{n} / \mathrm{a}$

comp

comp

comp
West Virginia

New Hampshire

New Hampshire

New Hampshire
Ker, 1980a

Whittaker et al., 1974

Ker, 1980a

Whittaker et al., 1974

Hocker and Earley, 1983

Ker, 1980a

Ribe, 1973

Whittaker et al., 1974

Young et al., 1980

Hocker and Earley, 1983

Ker, $1980 \mathrm{a}$

Ribe, 1973

Young et al., 1980

Hocker and Earley, 1983

Ker, 1980a

Ribe, 1973

Whittaker et al., 1974

Young et al.. 1980

Brenneman et al., 1978

Hocker and Earley, 1983

Hocker and Earley, 1983

Hocker and Earley, 1983
Birch, grey ( Betula populifolia Marsh.)

$\begin{array}{llllrllllll}\text { AB: } & 0.1218 & 2.3123 & 1-23 & 44 & 0.990 & 0.141 & \ln & 1.010 & \mathrm{n} / \mathrm{a} & \text { Nova Scotia } \\ & 0.1564 & 2.3146 & 3-24^{\mathrm{a}} & 30 & \mathrm{n} / \mathrm{a} & \mathrm{n} / \mathrm{a} & \mathrm{calc} & \mathrm{n} / \mathrm{a} & \text { comp } & \text { Maine } \\ \mathrm{SW}: & 0.0670 & 2.4240 & 1-23 & 44 & 0.990 & 0.141 & \ln & 1.010 & \mathrm{n} / \mathrm{a} & \text { Nova Scotia } \\ \mathrm{SB}: & 0.0185 & 2.2080 & 1-23 & 44 & 0.970 & 0.243 & \ln & 1.030 & \mathrm{n} / \mathrm{a} & \text { Nova Scotia } \\ \mathrm{ST}: & 0.0956 & 2.3600 & 2-8 & 5 & 0.991 & 0.162 & \ln & 1.013 & \mathrm{comp} & \text { New Hampshire } \\ & 0.0854 & 2.3875 & 1-23 & 44 & 0.990 & 0.141 & \ln & 1.010 & \mathrm{n} / \mathrm{a} & \text { Nova Scotia } \\ & 0.0857 & 2.5139 & 3-15 & 30 & 0.988 & \mathrm{n} / \mathrm{a} & \log & \mathrm{n} / \mathrm{a} & \mathrm{comp} & \text { Maine } \\ & 0.0826 & 2.5299 & 3-24^{\mathrm{a}} & 30 & 0.994 & \mathrm{n} / \mathrm{a} & \ln & \mathrm{n} / \mathrm{a} & \text { comp } & \text { Maine } \\ \text { FL: } & 0.0050 & 2.4100 & 2-8 & 5 & 0.942 & 0.429 & \ln & 1.096 & \text { comp } & \text { New Hampshire } \\ & 0.0129 & 1.7477 & 1-23 & 44 & 0.900 & 0.341 & \ln & 1.060 & \mathrm{n} / \mathrm{a} & \text { Nova Scotia } \\ & 0.0162 & 1.6376 & 3-15 & 30 & 0.871 & \mathrm{n} / \mathrm{a} & \log & \mathrm{n} / \mathrm{a} & \text { comp } & \text { Maine } \\ & 0.0414 & 1.2276 & 3-24 & 30 & 0.812 & \mathrm{n} / \mathrm{a} & \ln & \mathrm{n} / \mathrm{a} & \text { comp } & \text { Maine } \\ \text { BR: } & 0.0169 & 2.3800 & 2-8 & 5 & 0.970 & 0.298 & \ln & 1.045 & \text { comp } & \text { New Hampshire } \\ & 0.0192 & 2.1922 & 1-23 & 44 & 0.910 & 0.415 & \ln & 1.090 & \mathrm{n} / \mathrm{a} & \text { Nova Scotia } \\ & 0.0674 & 1.6163 & 3-15 & 30 & 0.988 & \mathrm{n} / \mathrm{a} & \log & \mathrm{n} / \mathrm{a} & \text { comp } & \text { Maine } \\ & 0.0669 & 1.6287 & 3-24 & 30 & 0.897 & \mathrm{n} / \mathrm{a} & \ln & \mathrm{n} / \mathrm{a} & \text { comp } & \text { Maine }\end{array}$

Birch, white (Betula papyrifera Marsh.)

$\begin{array}{clll}\text { AB: } & 0.0775 & 2.4800 & 2-8 \\ & 0.1347 & 2.3634 & 1-34 \\ 0.1074 & 2.4313 & 3-33 \\ 0.1545 & 2.3064 & 0-33 \\ & & \\ & 0.3154 & 1.7284 & 0-15 \\ 0.1182 & 2.4287 & 5-32 \\ 0.0882 & 2.5620 & 0-30 \\ 0.0612 & 2.6634 & 3-51^{\mathrm{a}} \\ \mathrm{SW}: & 0.0682 & 2.3600 & 2-8 \\ 0.0125 & 3.0221 & 1-34 \\ 0.0631 & 2.4931 & 3-33 \\ 0.0739 & 2.3982 & 0-33\end{array}$

\section{4 \\ 37}

$0.990 \quad 0.215$

$\begin{array}{lll}45 & 0.990 & 0.141\end{array}$

$\begin{array}{lll}196 & 0.978 & 24.50\end{array}$

$\begin{array}{ll}\log & n / a \\ \ln & 1.023 \\ \ln & 1.010 \\ \text { abs } & n / a \\ & \\ \log & n / a \\ \text { calc } & n / a \\ \text { abs } & n / a \\ \text { calc } & n / a \\ \log & n / a \\ \ln & 1.015 \\ \ln & 1.010 \\ \text { abs } & n / a\end{array}$

$\begin{array}{lll}21 & 0.919 & n / a\end{array}$

$52 \mathrm{n} / \mathrm{a} \quad \mathrm{n} / \mathrm{a}$

$\begin{array}{lll}204 & 0.990 & 10.50\end{array}$

51 n/a n/a

$240.941 \quad n / a$

$\begin{array}{lll}29 & 0.983 & 0.172\end{array}$

$\begin{array}{lll}45 & 0.990 & 0.141\end{array}$

$\begin{array}{lll}196 & 0.974 & 17.50\end{array}$ $\mathrm{n} / \mathrm{a}$

$\mathrm{n} / \mathrm{a}$

$\mathrm{n} / \mathrm{a}$

comp

New Brunswick

Nova Scotia

Nova Scotia

New Brunswick,

Nova Scotia

n/a New Brunswick

17(50) Upper Great Lakes

comp Canada-US ${ }^{\mathrm{c}}$

comp Maine

$\mathrm{n} / \mathrm{a} \quad$ New Brunswick

$n / a \quad$ Nova Scotia

$n / a$

comp
Nova Scotia

New Brunswick, Nova Scotia
Ker, 1980b

Young et al., 1980

Ker, 1980b

Ker, 1980b

Hocker and Earley, 1983

Ker, 1980b

Ribe, 1973

Young et al., 1980

Hocker and Earley, 1983

Ker, 1980b

Ribe, 1973

Young et al., 1980

Hocker and Earley, 1983

Ker, 1980b

Ribe, 1973

Young et al., 1980

Baskerville, 1965

Freedman et al., 1982

Ker, 1980b

Ker, 1984

MacLean and Wein, 1976

Perala and Alban, 1994

Schmitt and Grigal, 1981

Young et al., 1980

Baskerville, 1965

Freedman et al., 1982

Ker, 1980b

Ker, 1984 


\begin{tabular}{|c|c|c|c|c|c|c|c|c|c|c|c|}
\hline & 0.0806 & 2.4077 & $5-32$ & 52 & $\mathrm{n} / \mathrm{a}$ & $n / a$ & calc & $\mathrm{n} / \mathrm{a}$ & $17(50)$ & Upper Great Lakes & Pcrala and Alban, 1994 \\
\hline & 0.1171 & 2.3330 & $0-30$ & 144 & 0.980 & 5.561 & abs & $\mathrm{n} / \mathrm{a}$ & comp & Canada-US : & Schmitt and Grigal, 1981 \\
\hline & 0.2840 & 2.6400 & $1-35$ & 74 & 0.990 & 2.880 & abs & $\mathrm{n} / \mathrm{a}$ & comp & British Columbia & Wang et al.. 1996 \\
\hline \multirow[t]{6}{*}{ SB: } & 0.0011 & 2.3500 & $2-8$ & 24 & 0.810 & $n / a$ & $\log$ & $\mathrm{n} / \mathrm{a}$ & $\mathrm{n} / \mathrm{a}$ & New Brunswick & Baskerville, 1965 \\
\hline & 0.0061 & 2.6627 & $1-34$ & 29 & 0.977 & 0.181 & ln & 1.017 & $\mathrm{n} / \mathrm{a}$ & Nova Scotia & Freedman et al.. 1982 \\
\hline & 0.0196 & 2.2795 & $3-33$ & 45 & 0.990 & 0.141 & $\ln$ & 1.010 & $n / a$ & Nova Scotia & Ker. $1980 \mathrm{~b}$ \\
\hline & 0.0220 & 2.2150 & $5-32$ & 53 & 0.981 & 0.172 & $\ln$ & 1.015 & $17 \operatorname{mn}(50)$ & Upper Great Lakes & Perala and Alban, 1994 \\
\hline & 0.0407 & 2.0150 & $0-30$ & 143 & 0.970 & 0.979 & abs & $\mathrm{n} / \mathrm{a}$ & comp & Canada-US : & Schmitt and Grigat. 1981 \\
\hline & 0.0370 & 2.1640 & $1-35$ & 74 & 0.957 & 0.380 & abs & $\mathrm{n} / \mathrm{a}$ & comp & British Columbia & Wang et al.. 1996 \\
\hline \multirow[t]{9}{*}{ ST: } & 0.0173 & 2.9650 & $1-34$ & 29 & 0.984 & 0.167 & $\ln$ & 1.014 & $n / a$ & Nova Scotia & Freedman et al., 1982 \\
\hline & 0.2044 & 2.1700 & $0-34$ & 18 & 0.995 & 0.279 & $\ln$ & 1.040 & comp & New Hampshire & Hocker and Earley. 1983 \\
\hline & 0.0815 & 2.4594 & $3-33$ & 45 & 0.990 & 0.141 & $\ln$ & 1.010 & $n / a$ & Nova Scotia & Ker, $1980 \mathrm{~b}$ \\
\hline & 0.0847 & 2.4029 & $0-33$ & 196 & 0.973 & 20.60 & abs & $\mathrm{n} / \mathrm{a}$ & comp & $\begin{array}{l}\text { New Brunswick, } \\
\text { Nova Scotia }\end{array}$ & \\
\hline & 0.0413 & 2.8770 & $5-8$ & 6 & 0.982 & 0.099 & $\ln$ & 1.005 & $\mathrm{n} / \mathrm{a}$ & New Hampshire & $\begin{array}{l}\text { Kinerson and } \\
\text { Bartholomew, } 1977\end{array}$ \\
\hline & 0.1472 & 1.8805 & $0-15$ & 21 & 0.934 & $\mathrm{n} / \mathrm{a}$ & $\log$ & $\mathrm{n} / \mathrm{a}$ & $\pi / a$ & New Brunswick & MacLean and Wein, 1976 \\
\hline & 0.0134 & 3.2640 & $3-15$ & 30 & 0.989 & $\mathrm{n} / \mathrm{a}$ & $\log$ & $n / a$ & comp & $\mathrm{Ma}$ & Ribe, 1973 \\
\hline & 0.0923 & 2.4800 & $0-30$ & 228 & 0.980 & 10.68 & abs & $\mathrm{n} / \mathrm{a}$ & comp & ida-US : & Schmitt and Grigal, 1981 \\
\hline & 0.0263 & 2.8968 & $3-51$ : & 51 & 0.990 & $\mathrm{n} / \mathrm{a}$ & $\ln$ & $n / a$ & comp & Maine & Young et al., 1980 \\
\hline \multirow[t]{10}{*}{ rL: } & 0.0016 & 2.9400 & $2-8$ & 25 & 0.810 & $\mathrm{n} / \mathrm{a}$ & $\log$ & $\mathrm{n} / \mathrm{a}$ & $\mathrm{n} / \mathrm{a}$ & New Brunswick & Baskervilie, 1965 \\
\hline & 0.0180 & 1.7139 & $1-34$ & 37 & 0.896 & 0.526 & $\ln$ & 1.148 & $\mathrm{n} / \mathrm{a}$ & Scotia & et al. 1982 \\
\hline & 0.0400 & 1.7700 & $0-34$ & 5 & 0.933 & 0.219 & $\ln$ & 1.024 & comp & Hampshire & ind Earley, 1983 \\
\hline & 0.0142 & 1.8735 & $3-33$ & 45 & 0.910 & 0.341 & $\ln$ & 1.060 & $\mathrm{n} / \mathrm{a}$ & Scotia & Ker, \\
\hline & 0.0394 & 1.6286 & $0-33$ & 196 & 0.861 & 0.030 & absw & $\mathrm{n} / \mathrm{a}$ & comp & $\begin{array}{l}\text { swick, } \\
\text { tia }\end{array}$ & 4 \\
\hline & 0.0132 & 1.9505 & $5-32$ & 54 & $\mathrm{n} / \mathrm{a}$ & $\mathrm{n} / \mathrm{a}$ & calc & $n / a$ & $17(50)$ & Upper Great Lakes & Perala and Alban, 1994 \\
\hline & 0.0295 & 1.7020 & $3-15$ & 30 & 0.889 & $\mathrm{n} / \mathrm{a}$ & $\log$ & $\mathrm{n} / \mathrm{a}$ & comp & Maine & Ribe, 1973 \\
\hline & 0.0010 & 3.0050 & $0-30$ & 168 & 0.890 & 1.457 & abs & $\mathrm{n} / \mathrm{a}$ & comp & $\mathrm{da} \cdot \mathrm{US}^{\mathrm{c}}$ & Schmitt and Grigal. 3981 \\
\hline & 0.0210 & 1.9450 & $1-35$ & 74 & 0.828 & 0.220 & abs & $\mathrm{n} / \mathrm{a}$ & comp & British Columbia & Wang et al.. 1996 \\
\hline & 0.0162 & 2.0494 & $3-51^{3}$ & 51 & 0.962 & $\mathrm{n} / \mathrm{a}$ & $\ln$ & $n / a$ & comp & Maine & Young et al., 1980 \\
\hline \multirow[t]{10}{*}{ BR: } & 0.0021 & 3.3000 & $2-8$ & 24 & 0.740 & $n / a$ & $\log$ & $n / a$ & $\mathrm{n} / \mathrm{a}$ & New Brunswick & Baskerville, 1965 \\
\hline & 0.2364 & 1.6429 & $1-34$ & 37 & 0.955 & 0.322 & $\ln$ & 1.053 & $\mathrm{n} / \mathrm{a}$ & Scotia & man et al., 1982 \\
\hline & 0.0215 & 2.3000 & $0-34$ & 18 & 0.979 & 0.617 & $\ln$ & 1.210 & comp & New Hampshire & Hocker and Earley, 1983 \\
\hline & 0.0117 & 2.5073 & $3-33$ & 45 & 0.920 & 0.457 & $\ln$ & 1.110 & $n / a$ & Nova Scotia & Ker, $1980 \mathrm{~b}$ \\
\hline & 0.0579 & 2.1458 & $0-33$ & 196 & 0.892 & 12.80 & $a b s$ & $\mathrm{n} / \mathrm{a}$ & comp & $\begin{array}{l}\text { New Brunswick, } \\
\text { Nova Scotia }\end{array}$ & Ker. 1984 \\
\hline & 0.0003 & 4.3680 & $5-8$ & 6 & 0.904 & 0.362 & $\ln$ & 1.068 & $\mathrm{n} / \mathrm{a}$ & New Hampshire & $\begin{array}{l}\text { Kinerson and } \\
\text { Bartholomew, } 1977\end{array}$ \\
\hline & 0.0507 & 1.7304 & $3-15$ & 30 & 0.988 & $0 / a$ & $10 \mathrm{~g}$ & $\mathrm{II} / \mathrm{a}$ & comup & & Ribe, 1973 \\
\hline & 0.0012 & 3.2750 & $0-30$ & 177 & 0.890 & 4.226 & abs & $\mathrm{n} / \mathrm{a}$ & comp & Canada-US" & Schmitt and Grigal. 1981 \\
\hline & 0.0194 & 2.2494 & $3-51^{*}$ & 51 & 0.949 & $\mathrm{n} / \mathrm{a}$ & $\ln$ & $\mathrm{n} / \mathrm{a}$ & comp & Maine & Young et al.. 1980 \\
\hline & 0.0020 & 2.9130 & $1-35$ & 74 & 0.859 & 0.520 & abs & $n / a$ & comp & British Columbia & Wang et al., 1996 \\
\hline
\end{tabular}

Birch, yellow (Betula alleghaniensis Britt.)

$\begin{array}{llllrllllll}\text { AB: } & 0.1540 & 2.3753 & 5-50 & 24 & 0.970 & \mathrm{n} / \mathrm{a} & \log & \mathrm{n} / \mathrm{a} & \mathrm{n} / \mathrm{a} & \text { West Virginia } \\ & 0.1188 & 2.4510 & 3-29 & 24 & 0.991 & 0.180 & \ln & 1.016 & \mathrm{n} / \mathrm{a} & \text { Nova Scotia } \\ & 0.1541 & 2.3666 & 1-27 & 50 & 0.992 & 0.141 & \ln & 1.010 & \mathrm{n} / \mathrm{a} & \text { New Brunswick } \\ & 0.0872 & 2.5870 & 5-21 & 9 & 0.993 & 0.128 & \ln & 1.008 & \mathrm{n} 7(50) & \text { Upper Great Lakes } \\ & 0.1684 & 2.4150 & 1-55^{\mathrm{a}} & 14 & 0.994 & 0.099 & \log & 1.011 & \mathrm{n} / \mathrm{a} & \text { New Hampshire } \\ & 0.1588 & 2.3376 & 3-66^{a} & 42 & \mathrm{n} / \mathrm{a} & \mathrm{n} / \mathrm{a} & \mathrm{calc} & \mathrm{n} / \mathrm{a} & \mathrm{comp} & \text { Maine } \\ \mathrm{SW}: & 0.0224 & 2.8627 & 3-29 & 17 & 0.946 & 0.260 & \mathrm{ln} & 1.034 & \mathrm{n} / \mathrm{a} & \text { Nova Scotia } \\ & 0.0866 & 2.4369 & 1-27 & 50 & 0.992 & 0.141 & \mathrm{ln} & 1.010 & \mathrm{n} / \mathrm{a} & \text { New Brunswick } \\ & 0.0548 & 2.6190 & 5-21 & 9 & 0.993 & 0.129 & \ln & 1.008 & 17(50) & \text { Upper Great Lakes } \\ & 0.1131 & 2.2950 & 1-55^{\mathrm{a}} & 14 & 0.994 & 0.087 & \log & 1.009 & \mathrm{n} / \mathrm{a} & \text { New Hampshire } \\ \mathrm{SB}: & 0.0046 & 2.7136 & 3-29 & 17 & 0.935 & 0.271 & \ln & 1.037 & \mathrm{n} / \mathrm{a} & \text { Nova Scotia } \\ & 0.0172 & 2.3086 & 1-27 & 50 & 0.980 & 0.199 & \ln & 1.020 & \mathrm{n} / \mathrm{a} & \text { New Brunswick }\end{array}$

Brenneman et al, 1978 Freedman et al.. 1982 Ker, 1980a Perala and Alban. 1994 Whittaker et al. 1974 Young et al, 1980 Freedman et al., 1982

Ker, 1980a Perala and Alban, 1994 Whittaker et al., 1974 Freedman et al.. 1982 Ker, 1980a 


\begin{tabular}{|c|c|c|c|c|c|c|c|c|c|c|}
\hline & 0.0145 & 2.4510 & $5-21$ & 9 & 0.985 & 0.181 & $\ln$ & 1.017 & $17(50)$ & Upper Great Lakes \\
\hline & 0.0252 & 2.1083 & $1-55^{\mathrm{a}}$ & 14 & 0.988 & 0.111 & $\log$ & 1.014 & $\mathrm{n} / \mathrm{a}$ & New Hampshirc \\
\hline \multirow[t]{6}{*}{ ST: } & 0.0269 & 2.8437 & $3-29$ & 17 & 0.946 & 0.258 & $\ln$ & 1.034 & $\mathrm{n} / \mathrm{a}$ & Nova Scotia \\
\hline & 0.0737 & 2.5600 & $1-12$ & 13 & 0.952 & 0.577 & $\ln$ & 1.181 & comp & New Hampshire \\
\hline & 0.1036 & 2.4200 & $1-27$ & 50 & 0.992 & 0.141 & $\ln$ & 1.010 & $\mathrm{n} / \mathrm{a}$ & New Brunswick \\
\hline & 0.0874 & 2.5330 & $3-15$ & 30 & 0.994 & $\mathrm{n} / \mathrm{a}$ & $\log$ & $\mathrm{n} / \mathrm{a}$ & comp & Maine \\
\hline & 0.1385 & 2.2683 & $1-55^{a}$ & 14 & 0.994 & 0.087 & $\log$ & 1.009 & $\mathrm{n} / \mathrm{a}$ & New Hampshire \\
\hline & 0.1085 & 2.3412 & $3-66^{a}$ & 42 & 0.939 & $\mathrm{n} / \mathrm{a}$ & $\ln$ & $\mathrm{n} / \mathrm{a}$ & comp & Maine \\
\hline \multirow[t]{6}{*}{ FL: } & 0.0064 & 2.1167 & $3-29$ & 24 & 0.961 & 0.322 & $\ln$ & 1.053 & $\mathrm{n} / \mathrm{a}$ & Nova Scotia \\
\hline & 0.0149 & 1.9900 & $1-12$ & 1 & 0.987 & 0.264 & ln & 1.035 & comp & New Hampshire \\
\hline & 0.0165 & 1.7241 & $1-27$ & 49 & 0.914 & 0.368 & In & 1.070 & $\mathrm{n} / \mathrm{a}$ & New Brunswick \\
\hline & 0.0070 & 2.0030 & $5-21$ & 9 & 0.785 & 0.632 & $\ln$ & 1.221 & $17(50)$ & Upper Great Lakes \\
\hline & 0.0158 & 1.9683 & $3-15$ & 30 & 0.900 & $\mathrm{n} / \mathrm{a}$ & $\log$ & $\mathrm{n} / \mathrm{a}$ & comp & Maine \\
\hline & 0.0155 & 1.9783 & $3-66^{a}$ & 42 & 0.963 & $\mathrm{n} / \mathrm{a}$ & $\ln$ & $\mathrm{n} / \mathrm{a}$ & comp & Maine \\
\hline \multirow[t]{6}{*}{ BR: } & 0.3507 & 1.5899 & $3-29$ & 24 & 0.919 & 0.360 & $\ln$ & 1.067 & $\mathrm{n} / \mathrm{a}$ & Nova Scotia \\
\hline & 0.0291 & 2.3500 & $1-12$ & 13 & 0.972 & 0.400 & $\ln$ & 1.083 & comp & New Hampshire \\
\hline & 0.0287 & 2.3585 & $1-27$ & 50 & 0.908 & 0.512 & $\ln$ & 1.140 & $\mathrm{n} / \mathrm{a}$ & New Brunswick \\
\hline & 0.0758 & 1.6179 & $3-15$ & 30 & 0.898 & $\mathrm{n} / \mathrm{a}$ & $\log$ & $\mathrm{n} / \mathrm{a}$ & comp & Maine \\
\hline & 0.0113 & 2.7995 & $1-55^{a}$ & 14 & 0.955 & 0.300 & $\log$ & 1.109 & $\mathrm{n} / \mathrm{a}$ & New Hampshire \\
\hline & 0.0216 & 2.3795 & $3-66^{a}$ & 42 & 0.921 & $\mathrm{n} / \mathrm{a}$ & $\ln$ & $\mathrm{n} / \mathrm{a}$ & comp & Maine \\
\hline
\end{tabular}

Cedar, eastern white (Thuja occidentalis L.)

$\begin{array}{lllllllllll}\text { AB: } & 0.1148 & 2.1439 & 2-30 & 46 & 0.991 & 0.141 & \ln & 1.010 & \mathrm{n} / \mathrm{a} & \text { New Brunswick } \\ & 0.0910 & 2.2340 & 4-31 & 20 & 0.990 & 0.125 & \ln & 1.008 & \mathrm{n} / \mathrm{a} & \text { Upper Great Lakes } \\ & 0.2305 & 1.9269 & 3-51^{\mathrm{a}} & 39 & \mathrm{n} / \mathrm{a} & \mathrm{n} / \mathrm{a} & \mathrm{calc} & \mathrm{n} / \mathrm{a} & \mathrm{comp} & \text { Maine } \\ \mathrm{SW}: & 0.0520 & 2.2804 & 2-30 & 47 & 0.989 & 0.141 & \ln & 1.010 & \mathrm{n} / \mathrm{a} & \text { New Brunswick } \\ & 0.0606 & 2.1491 & 4-31 & 20 & \mathrm{n} / \mathrm{a} & \mathrm{n} / \mathrm{a} & \mathrm{calc} & \mathrm{n} / \mathrm{a} & \mathrm{n} / \mathrm{a} & \text { Upper Great Lakes } \\ \mathrm{SB}: & 0.0094 & 2.2228 & 2-30 & 47 & 0.987 & 0.199 & \ln & 1.020 & \mathrm{n} / \mathrm{a} & \text { New Brunswick } \\ & 0.0114 & 2.1240 & 431 & 20 & \mathrm{n} / \mathrm{a} & \mathrm{n} / \mathrm{a} & \mathrm{calc} & \mathrm{n} / \mathrm{a} & \mathrm{n} / \mathrm{a} & \text { Upper Great Lakes } \\ \mathrm{ST}: & 0.0618 & 2.2706 & 2-30 & 47 & 0.990 & 0.141 & \ln & 1.010 & \mathrm{n} / \mathrm{a} & \text { New Brunswick } \\ & 0.0832 & 2.1300 & 3-51^{\mathrm{a}} & 39 & 0.991 & \mathrm{n} / \mathrm{a} & \ln & \mathrm{n} / \mathrm{a} & \mathrm{comp} & \text { Maine } \\ \mathrm{FL}: & 0.0350 & 1.6206 & 2-30 & 46 & 0.856 & 0.280 & \ln & 1.040 & \mathrm{n} / \mathrm{a} & \text { New Brunswick } \\ & 0.0100 & 2.3030 & 4-31 & 20 & 0.893 & 0.450 & \ln & 1.107 & \mathrm{n} / \mathrm{a} & \text { Upper Great Lakes } \\ & 0.1496 & 1.3352 & 3-51^{\mathrm{a}} & 39 & 0.961 & \mathrm{n} / \mathrm{a} & \ln & \mathrm{n} / \mathrm{a} & \mathrm{comp} & \text { Maine } \\ \mathrm{BR}: & 0.0472 & 1.7434 & 2-30 & 46 & 0.863 & 0.312 & \ln & 1.050 & \mathrm{n} / \mathrm{a} & \text { New Brunswick } \\ & 0.0480 & 1.9110 & 3-51^{\mathrm{a}} & 39 & 0.973 & \mathrm{n} / \mathrm{a} & \ln & \mathrm{n} / \mathrm{a} & \mathrm{comp} & \text { Maine }\end{array}$

Cedar, western red (Thuja plicata Donn)

$\begin{array}{lllllllll}\text { SW: } & 0.1019 & 2.3000 & 12-47 & 10 & 0.960 & 0.225 & \ln & 1.026 \\ & 0.1022 & 2.0880 & 12-61 & 21 & 0.860 & 0.361 & \ln & 1.067 \\ \text { SB: } & 0.0104 & 2.1980 & 12-47 & 10 & 0.970 & 0.203 & \ln & 1.021 \\ & 0.0171 & 1.9990 & 12-61 & 21 & 0.880 & 0.304 & \ln & 1.047 \\ \text { ST: } & 0.3721 & 1.2928 & 0-12 & 25 & 0.854 & 0.602 & \ln & 1.199 \\ \text { FL: } & 0.2805 & 1.3313 & 0-68 & 22 & 0.924 & 0.582 & \ln & 1.184 \\ & 0.0147 & 2.1910 & 12-47 & 10 & 0.860 & 0.439 & \ln & 1.101 \\ & 0.0494 & 1.9220 & 12-61 & 21 & 0.930 & 0.226 & \ln & 1.026 \\ \text { BR: } & 0.1379 & 1.5986 & 0-68 & 22 & 0.923 & 0.702 & \ln & 1.279 \\ & 0.0599 & 1.8020 & 1247 & 10 & 0.800 & 0.452 & \ln & 1.108 \\ & 0.0277 & 2.1390 & 12-61 & 21 & 0.910 & 0.281 & \ln & 1.040\end{array}$

Cedar, yellow (Chamaecyparis nootkatensis (D. Don) Spach)

$\begin{array}{llllllllll}\text { AB: } & 0.2498 & 2.1118 & 18-60 & 4 & 0.992 & 0.123 & \ln & 1.008 & \mathrm{n} / \mathrm{a} \\ \text { SW: } & 0.1323 & 2.1989 & 18-60 & 4 & 0.982 & 0.197 & \ln & 1.020 & \mathrm{n} / \mathrm{a} \\ \text { SB: } & 0.0175 & 2.0829 & 18-60 & 4 & 0.964 & 0.268 & \ln & 1.037 & \mathrm{n} / \mathrm{a} \\ \mathrm{ST}: & 0.1492 & 2.1889 & 18-60 & 4 & 0.982 & 0.199 & \ln & 1.020 & \mathrm{n} / \mathrm{a} \\ \mathrm{FI}: & 0.1258 & 1.6164 & 18-60 & 4 & 0.926 & 0.304 & \ln & 1.047 & \mathrm{n} / \mathrm{a} \\ \text { BR: } & 0.0293 & 2.1168 & 18-60 & 4 & 0.980 & 0.200 & \ln & 1.020 & \mathrm{n} / \mathrm{a}\end{array}$

$\begin{array}{ll}\text { n/a } & \text { British Columbia } \\ \text { comp } & \text { British Columbia } \\ \text { n/a } & \text { British Columbia } \\ \text { comp } & \text { British Columbia } \\ \text { comp } & \text { Idaho, Montana } \\ \text { comp } & \text { Idaho, Montana } \\ \text { n/a } & \text { British Columbia } \\ \text { comp } & \text { British Columbia } \\ \text { comp } & \text { Idaho, Montana } \\ \text { n/a } & \text { British Columbia } \\ \text { comp } & \text { British Columbia }\end{array}$

British Columbia British Columbia British Columbia British Columbia British Columbia British Columbia
Perala and Alban, 1994

Whittaker et al., 1974

Freedman et al., 1982

Hocker and Earley, 1983

Ker, 1980 a

Ribe, 1973

Whittaker et al., 1974

Young et al., 1980

Freedman et al., 1982

Hocker and Earley, 1983

Ker, 1980a

Perala and Alban, 1994

Ribe, 1973

Young et al., 1980

Freedman et al., 1982

Hocker and Earley, 1983

Ker, 1980a

Ribe, 1973

Whittaker et al., 1974

Young et al., 1980
Ker, 1980a

Perala and Alban, 1994

Young et al., 1980

Ker, 1980a

Perala and Alban, 1994

Ker, 1980a

Perala and Alban, 1994

Ker, 1980a

Young et al., 1980

Ker, 1980a

Perala and Alban, 1994

Young et al., 1980

Ker, 1980a

Young et al., 1980
Feller, 1992

Feller, 1992

Feller, 1992

Feller, 1992

Brown, $1978^{b}$

Brown. $1978^{\text {b }}$

Feller, 1992

Feller, 1992

Brown, $1978^{b}$

Feller, 1992

Feller, 1992

Krumlik, $1974^{\mathrm{b}}$ Krumlik, $1974^{\text {b }}$ Krumlik, $1974^{\text {b }}$ Krumlik, $1974^{\text {b }}$ Krumlik, $1974^{\text {b }}$ Krumlik, $1974^{\text {b }}$ 
Cherry, black (Prunus serotina Ehrh.)

$\begin{array}{llllllllllll}\mathrm{AB}: & 0.0716 & 2.6174 & 5-50 & 26 & 0.990 & \mathrm{n} / \mathrm{a} & \log & \mathrm{n} / \mathrm{a} & \mathrm{n} / \mathrm{a} \quad \text { West Virginia } & \text { Brenneman et al., } 1978 \\ & 0.1225 & 2.4253 & 5-40^{a} & 19 & 0.994 & 20.41 & \text { abs } & \mathrm{n} / \mathrm{a} & 22.3(50) \text { West Virginia } & \text { Wiant et al.. 1977 } \mathrm{d} .\end{array}$

Cherry, choke (Prunus virginiana L.)

$\begin{array}{lllllllllll}\mathrm{AB}: & 0.2643 & 1.7102 & 3-15^{\mathrm{a}} & 16 & \mathrm{n} / \mathrm{a} & \mathrm{n} / \mathrm{a} & \mathrm{calc} & \mathrm{n} / \mathrm{a} & \mathrm{comp} & \text { Maine } \\ \mathrm{ST}: & 0.1161 & 2.0038 & 3-8 & 16 & 0.848 & \mathrm{n} / \mathrm{a} & \log & \mathrm{n} / \mathrm{a} & \text { comp } & \text { Maine } \\ & 0.1178 & 1.9936 & 3-15^{\mathrm{a}} & 16 & 0.918 & \mathrm{n} / \mathrm{a} & \ln & \mathrm{n} / \mathrm{a} & \text { comp } & \text { Maine } \\ \mathrm{FL}: & 0.0327 & 1.3307 & 3-8 & 16 & 0.595 & \mathrm{n} / \mathrm{a} & \log & \mathrm{n} / \mathrm{a} & \mathrm{comp} & \text { Maine } \\ & 0.0319 & 1.3356 & 3-15^{\mathrm{a}} & 16 & 0.749 & \mathrm{n} / \mathrm{a} & \ln & \mathrm{n} / \mathrm{a} & \mathrm{comp} & \text { Maine } \\ \mathrm{BR}: & 0.1149 & 1.2191 & 3-8 & 16 & 0.560 & \mathrm{n} / \mathrm{a} & \log & \mathrm{n} / \mathrm{a} & \text { comp } & \text { Maine } \\ & 0.1196 & 1.1932 & 3-15^{\mathrm{a}} & 16 & 0.742 & \mathrm{n} / \mathrm{a} & \ln & \mathrm{n} / \mathrm{a} & \text { comp } & \text { Maine }\end{array}$

Cherry, fire (Prunus pensylvanica L.f.)

$\begin{array}{lllllllllll}\text { AB: } & 0.2159 & 1.7041 & 0-10 & 17 & 0.859 & \mathrm{n} / \mathrm{a} & \log & \mathrm{n} / \mathrm{a} & \mathrm{n} / \mathrm{a} & \text { New Brunswick } \\ \mathrm{ST}: & 0.1259 & 1.7772 & 0-10 & 17 & 0.881 & \mathrm{n} / \mathrm{a} & \log & \mathrm{n} / \mathrm{a} & \mathrm{n} / \mathrm{a} & \text { New Brunswick }\end{array}$

Young et al., 1980

Ribe, 1973

Young et al. 1980

Ribe, 1973

Young et al.. 1980

Ribe, 197.3

Young et al.. 1980

MacLean and Wein, 1976

MacLean and Wein, 1976

Cherry, pin ( Prunus pensylmanica $\mathrm{I}$..)

$\begin{array}{lllllllllll}\text { AB: } & 0.1556 & 2.1948 & 3-24^{a} & 30 & \mathrm{n} / \mathrm{a} & \mathrm{n} / \mathrm{a} & \mathrm{calc} & \mathrm{n} / \mathrm{a} & \text { comp } & \text { Maine } \\ \mathrm{ST}: & 0.0783 & 2.4000 & 1-14 & 12 & 0.972 & 0.461 & \ln & 1.112 & \text { comp } & \text { New Hampshire } \\ & 0.0957 & 2.2988 & 3-15 & 30 & 0.982 & \mathrm{11} / \mathrm{a} & \log & \mathrm{n} / \mathrm{a} & \text { comp } & \text { Mainc } \\ & 0.0951 & 2.2988 & 3-24^{\mathrm{a}} & 30 & 0.991 & \mathrm{n} / \mathrm{a} & \ln & \mathrm{n} / \mathrm{a} & \text { comp } & \text { Maine } \\ \mathrm{FL}: & 0.0198 & 1.9784 & 3-15 & 30 & 0.904 & \mathrm{n} / \mathrm{a} & \log & \mathrm{n} / \mathrm{a} & \text { comp } & \text { Maine } \\ & 0.0203 & 2.0380 & 3-24^{a} & 30 & 0.783 & \mathrm{n} / \mathrm{a} & \ln & \mathrm{n} / \mathrm{a} & \text { comp } & \text { Maine } \\ \mathrm{BR}: & 0.0124 & 2.4300 & 1-14 & 12 & 0.934 & 0.725 & \ln & 1.301 & \text { comp } & \text { New Hampshire } \\ & 0.0441 & 1.8755 & 3-15 & 30 & 0.871 & \mathrm{n} / \mathrm{a} & \log & \mathrm{n} / \mathrm{a} & \text { comp } & \text { Maine } \\ & 0.0406 & 1.9197 & 3-24^{a} & 30 & 0.932 & \mathrm{n} / \mathrm{a} & \ln & \mathrm{n} / \mathrm{a} & \text { comp } & \text { Maine }\end{array}$

Chinkapin, golden (Castanopsis chrysophylla (Dougl.) A. DC.)

$\begin{array}{lllllllllll}\text { SW: } & 0.0240 & 2.6580 & 6-36 & 19 & 0.980 & 0.210 & \ln & 1.022 & \mathrm{n} / \mathrm{a} & \text { Oregon } \\ \text { SB: } & 0.0026 & 2.9890 & 6-36 & 19 & 0.970 & 0.261 & \ln & 1.035 & \mathrm{n} / \mathrm{a} & \text { Oregon } \\ \text { FL: } & 0.0401 & 1.6930 & 6-36 & 19 & 0.810 & 0.430 & \ln & 1.097 & \mathrm{n} / \mathrm{a} & \text { Oregon } \\ & 0.0214 & 1.8042 & 3-60 & 30 & 0.944 & 0.359 & \ln & 1.067 & \mathrm{comp} & \text { Oregon, California } \\ \text { BR: } & 0.0092 & 2.5760 & 6-36 & 19 & 0.890 & 0.473 & \ln & 1.119 & \mathrm{n} / \mathrm{a} & \text { Oregon } \\ & 0.0128 & 2.3448 & 3-60 & 30 & 0.942 & 0.477 & \ln & 1.120 & \mathrm{comp} & \text { Oregon, California }\end{array}$

Gholz et al.. 1979

Gholz et al. 1979

Gholz et al., 1979

Snell and Little. $1983^{\text {b }}$

Gholz et al.. 1979

Snell and Little. $1983^{\text {b }}$

Elm, American (Ulmus americana L..)

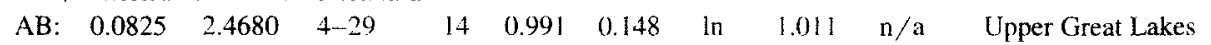

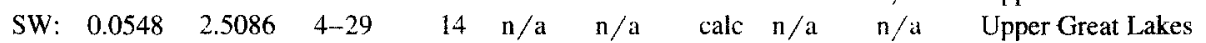

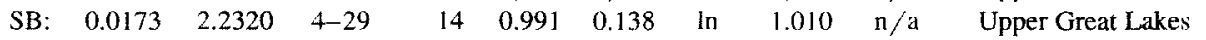

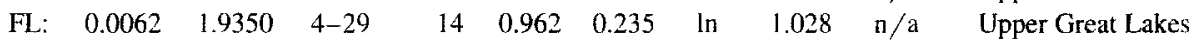

Perala and Alban, 1994

Perala and Alban, 1994

Perala and Aiban, 1994

Perala and Alban, 1994

Fir, balsam ( Abies balsamea (L.) Mill.)

$\begin{array}{lllllllllll}\text { AB: } & 0.0523 & 2.5300 & 3-25 & 101 & 0.923 & \mathrm{n} / \mathrm{a} & \log & \mathrm{n} / \mathrm{a} & \mathrm{n} / \mathrm{a} & \text { New Brunswick } \\ & 0.1075 & 2.3263 & 3-28 & 30 & 0.987 & 0.197 & \ln & 1.020 & \mathrm{n} / \mathrm{a} & \text { Nova Scotia } \\ 0.2575 & 2.0543 & 3-40 & 40 & 0.990 & 0.278 & \ln & 1.039 & \mathrm{n} / \mathrm{a} & \text { Ontario } \\ 0.0690 & 2.4975 & 3-40 & 40 & 0.970 & 0.123 & \ln & 1.008 & \mathrm{n} / \mathrm{a} & \text { Ontario } \\ 0.1598 & 2.1283 & 2-32 & 50 & 0.970 & 0.243 & \ln & 1.030 & \mathrm{n} / \mathrm{a} & \text { Nova Scotia } \\ 0.1746 & 2.1555 & 0-36 & 196 & 0.982 & 19.60 & \mathrm{abs} & \mathrm{n} / \mathrm{a} & \mathrm{comp} & \text { New Brunswick. } \\ & & & & & & & & & \text { Nova Scotia } \\ & 0.3908 & 1.6217 & 0-20 & 20 & 0.812 & \mathrm{n} / \mathrm{a} & \log & \mathrm{n} / \mathrm{a} & \mathrm{n} / \mathrm{a} & \text { New Brunswick } \\ & 0.0705 & 2.4970 & 4-34 & 60 & 0.986 & 0.175 & \ln & 1.015 & \mathrm{n} / \mathrm{a} & \text { Upper Great Lakes } \\ 0.0877 & 2.4017 & 3-51 & 95 & \mathrm{n} / \mathrm{a} & \mathrm{n} / \mathrm{a} & \mathrm{calc} & \mathrm{n} / \mathrm{a} & \mathrm{comp} & \text { Maine } \\ \mathrm{SW}: & 0.0625 & 2.2800 & 3-25 & 101 & 0.923 & \mathrm{n} / \mathrm{a} & \log & \mathrm{n} / \mathrm{a} & \mathrm{n} / \mathrm{a} & \text { New Brunswick } \\ & 0.0444 & 2.3977 & 3-28 & 30 & 0.992 & 0.156 & \ln & 1.012 & \mathrm{n} / \mathrm{a} & \text { Nova Scotia } \\ 0.0407 & 2.4228 & 2-32 & 50 & 0.980 & 0.199 & \ln & 1.020 & \mathrm{n} / \mathrm{a} & \text { Nova Scotia } \\ 0.0645 & 2.2962 & 0-36 & 198 & 0.979 & 12.80 & \mathrm{abs} & \mathrm{n} / \mathrm{a} & \text { comp } & \text { New Brunswick, } \\ & & & & & & & & & \text { Nova Scotia }\end{array}$

Baskerville. 1965

Freedman et al.. 1982

Honer, $1971^{\circ}$

Honer, 197! ${ }^{i}$

Ker, 1980b

Ker. 1984

Maclean and Wein, 1976

Perala and Alban, 1994

Young et al., 1980.

Baskerville, 1965

Freedman et at. 1982

Ker, $1980 \mathrm{~b}$

Ker, 1984

Hocker and Earley. 1983

\section{3}

Young ef al.. 1980

Young et al.. 1980

and Earley. 1983

Young et al., 1980 
M.T. Ter-Mikaelian, M. D. Korzukhin / Forest Ecology and Management 97 (1997) 1-24

\begin{tabular}{|c|c|c|c|c|c|c|c|c|c|c|c|}
\hline & 0.0302 & 2.5231 & $4-34$ & 59 & $\mathrm{n} / \mathrm{a}$ & $\mathrm{n} / \mathrm{a}$ & calc & $\mathrm{n} / \mathrm{a}$ & $\mathrm{n} / \mathrm{a}$ & Upper Great Lakes & Perala and Alban, 1994 \\
\hline \multirow[t]{5}{*}{ SB: } & 0.0055 & 2.4700 & $3-25$ & 101 & 0.903 & $\mathrm{n} / \mathrm{a}$ & $\log$ & $\mathrm{n} / \mathrm{a}$ & $\mathrm{n} / \mathrm{a}$ & New Brunswick & Baskerville, 1965 \\
\hline & 0.0174 & 2.1601 & $3-28$ & 30 & 0.982 & 0.219 & $\ln$ & 1.024 & $\mathrm{n} / \mathrm{a}$ & Nova Scotia & Freedman et al., 1982 \\
\hline & 0.0160 & 2.1140 & $3-40$ & 40 & 0.975 & 0.558 & $\ln$ & 1.168 & $\mathrm{n} / \mathrm{a}$ & Ontario & Honer, $1971^{e}$ \\
\hline & 0.0120 & 2.2391 & $2-32$ & 50 & 0.950 & 0.341 & $\ln$ & 1.060 & $\mathrm{n} / \mathrm{a}$ & Nova Scotia & Ker, $1980 b$ \\
\hline & 0.0065 & 2.3832 & $4-34$ & 60 & $\mathrm{n} / \mathrm{a}$ & $\mathrm{n} / \mathrm{a}$ & calc & $\mathrm{n} / \mathrm{a}$ & $\mathrm{n} / \mathrm{a}$ & Upper Great Lakes & Perala and Alban, 1994 \\
\hline \multirow[t]{5}{*}{ ST: } & 0.0607 & 2.3524 & $3-28$ & 30 & 0.992 & 0.156 & $\ln$ & 1.012 & $n / a$ & Nova Scotia & Freedman et al., 1982 \\
\hline & 0.0525 & 2.3932 & $2-32$ & 50 & 0.980 & 0.199 & $\ln$ & 1.020 & $\mathrm{n} / \mathrm{a}$ & Nova Scotia & Ker, 1980b \\
\hline & 0.0671 & 2.3381 & $0-36$ & 198 & 0.978 & 15.40 & abs & $\mathrm{n} / \mathrm{a}$ & comp & $\begin{array}{l}\text { New Brunswick, } \\
\text { Nova Scotia }\end{array}$ & Ker, 1984 \\
\hline & 0.1301 & 1.8728 & $0-20$ & 20 & 0.800 & $\mathrm{n} / \mathrm{a}$ & $\log$ & $\mathrm{n} / \mathrm{a}$ & $\mathrm{n} / \mathrm{a}$ & New Brunswick & MacLean and Wein, 1976 \\
\hline & 0.0679 & 2.4117 & $3-51^{\text {a }}$ & 95 & 0.995 & $\mathrm{n} / \mathrm{a}$ & $\ln$ & $\mathrm{n} / \mathrm{a}$ & comp & Maine & Young et al., 1980 \\
\hline \multirow[t]{7}{*}{ FL: } & 0.0013 & 3.2100 & $3-25$ & 101 & 0.960 & $\mathrm{n} / \mathrm{a}$ & $\log$ & $\mathrm{n} / \mathrm{a}$ & $\mathrm{n} / \mathrm{a}$ & New Brunswick & Baskerville, 1965 \\
\hline & 0.0153 & 2.3367 & $3-28$ & 30 & 0.916 & 0.524 & $\ln$ & 1.147 & $n / a$ & Nova Scotia & Freedman et al., 1982 \\
\hline & 0.1336 & 1.7853 & $3-40$ & 40 & 0.966 & 0.390 & $\ln$ & 1.079 & $\mathrm{n} / \mathrm{a}$ & Ontario & Honer, $1971^{\mathrm{e}}$ \\
\hline & 0.0617 & 1.6737 & $2-32$ & 50 & 0.900 & 0.312 & $\ln$ & 1.050 & $n / a$ & Nova Scotia & Ker, $1980 b$ \\
\hline & 0.0998 & 1.6421 & $0-36$ & 196 & 0.848 & 0.080 & absw & $\mathrm{n} / \mathrm{a}$ & comp & $\begin{array}{l}\text { New Brunswick, } \\
\text { Nova Scotia }\end{array}$ & Ker, 1984 \\
\hline & 0.0230 & 2.2565 & $4-34$ & 59 & $\mathrm{n} / \mathrm{a}$ & $\mathrm{n} / \mathrm{a}$ & calc & $\mathrm{n} / \mathrm{a}$ & $\mathrm{n} / \mathrm{a}$ & Upper Great Lakes & Perala and Alban, 1994 \\
\hline & 0.0089 & 2.4506 & $3-51^{a}$ & 95 & 0.945 & $\mathrm{n} / \mathrm{a}$ & $\ln$ & $\mathrm{n} / \mathrm{a}$ & comp & Maine & Young et al., 1980 \\
\hline \multirow[t]{6}{*}{ BR: } & 0.0011 & 3.2200 & $3-25$ & 101 & 0.903 & $n / a$ & log & $11 / d$ & $\mathrm{II} / \mathrm{a}$ & New Bruiswick & Baskerville, 1965 \\
\hline & 0.0129 & 2.4263 & $3-28$ & 30 & 0.924 & 0.517 & $\ln$ & 1.143 & $\mathrm{n} / \mathrm{a}$ & Nova Scotia & Freedman et al., 1982 \\
\hline & 0.0690 & 2.0964 & $3-40$ & 40 & 0.979 & 0.385 & $\ln$ & 1.077 & $\mathrm{n} / \mathrm{a}$ & Ontario & Honer, $1971^{c}$ \\
\hline & 0.0721 & 1.7793 & $2-32$ & 50 & 0.890 & 0.312 & $\ln$ & 1.050 & $\mathrm{n} / \mathrm{a}$ & Nova Scotia & Ker, $1980 \mathrm{~b}$ \\
\hline & 0.0909 & 1.8405 & $0-36$ & 196 & 0.857 & 10.90 & abs & $\mathrm{n} / \mathrm{a}$ & comp & $\begin{array}{l}\text { New Brunswick, } \\
\text { Nova Scotia }\end{array}$ & Ker, 1984 \\
\hline & 0.0050 & 2.4605 & $3-51^{a}$ & 95 & 0.949 & $\mathrm{n} / \mathrm{a}$ & $\ln$ & $\mathrm{n} / \mathrm{a}$ & comp & Maine & Young et al., 1980 \\
\hline
\end{tabular}

Fir, Douglas ( $P$ seudotsuga menziesii (Mirb.) Franco)

\begin{tabular}{|c|c|c|c|c|c|c|c|c|c|c|c|}
\hline $\mathrm{AB}:$ & 0.0808 & 2.5282 & $5-54$ & 60 & 0.972 & 0.279 & $\ln$ & 1.040 & $\mathrm{n} / \mathrm{a}$ & British Columbia & Marshall and Wang, $1995^{b}$ \\
\hline \multirow[t]{5}{*}{ SW: } & 0.0158 & 2.8950 & $5-56$ & 10 & 0.960 & 0.493 & $\ln$ & 1.129 & good & British Columbia & Feller, 1992 \\
\hline & 0.0181 & 2.9270 & $6-29$ & 8 & 0.980 & 0.243 & $\ln$ & 1.030 & poor & British Columbia & Feller, 1992 \\
\hline & 0.0151 & 2.8270 & $5-64$ & 46 & 0.970 & 0.269 & $\ln$ & 1.037 & c.good & British Columbia & Feller, 1992 \\
\hline & 0.0137 & 2.8660 & $5-35$ & 42 & 0.960 & 0.280 & $\ln$ & 1.040 & c.poor & British Columbia & Feller, 1992 \\
\hline & 0.0113 & 2.5772 & $5-54$ & 60 & 0.958 & 0.350 & In & 1.063 & $\mathrm{n} / \mathrm{a}$ & British Columbia & Marshall and Wang, $1995^{\circ}$ \\
\hline \multirow[t]{6}{*}{ SB: } & 0.0023 & 2.8530 & $5-56$ & 10 & 0.970 & 0.472 & $\ln$ & 1.118 & good & British Columbia & Feller, 1992 \\
\hline & 0.0045 & 2.8530 & $6-29$ & 8 & 0.980 & 0.249 & $\ln$ & 1.031 & poor & British Columbia & Feller, 1992 \\
\hline & 0.0043 & 2.6590 & $5-64$ & 46 & 0.960 & 0.297 & $\ln$ & 1.045 & c.good & British Columbia & Feller, 1992 \\
\hline & 0.0041 & 2.6780 & $5-35$ & 42 & 0.940 & 0.308 & $\ln$ & 1.049 & c.poor & British Columbia & Feller, 1992 \\
\hline & 0.0127 & 2.4300 & $2-162$ & 99 & 0.990 & 0.322 & $\ln$ & 1.053 & comp & Oregon, Washington & Gholz et al., 1979 \\
\hline & 0.0336 & 2.6518 & $5-54$ & 60 & 0.956 & 0.369 & $\ln$ & 1.070 & $\mathrm{n} / \mathrm{a}$ & British Columbia & Marshall and Wang, $1995^{b}$ \\
\hline \multirow[t]{3}{*}{ ST: } & 0.2168 & 1.7236 & $1-11$ & 22 & 0.897 & 0.419 & $\ln$ & 1.092 & comp & Idaho, Montana & Brown, $1978^{h}$ \\
\hline & 0.0456 & 2.5951 & $2-162$ & 99 & 0.990 & 0.310 & $\ln$ & 1.049 & comp & Oregon, Washington & Gholz et al., 1979 \\
\hline & 0.0451 & 2.6343 & $5-54$ & 60 & 0.958 & 0.357 & $\ln$ & 1.066 & $\mathrm{n} / \mathrm{a}$ & British Columbia & Marshall and Wang, $1995^{b}$ \\
\hline \multirow[t]{7}{*}{ FI : } & 0.3021 & 1.3076 & $1-86$ & 22 & 0.932 & 0.541 & $\ln$ & 1.158 & comp & Jdaho, Montana & Brown, $1978^{b}$ \\
\hline & 0.2897 & 1.2850 & $5-56$ & 10 & 0.960 & 0.220 & $\ln$ & 1.024 & good & British Columbia & Feller, 1992 \\
\hline & 0.1315 & 1.4600 & $6-29$ & 8 & 0.870 & 0.339 & $\ln$ & 1.059 & poor & British Columbia & Feller, 1992 \\
\hline & 0.1105 & 1.6360 & $5-64$ & 43 & 0.870 & 0.364 & $\ln$ & 1.068 & c.good & British Columbia & Feller, 1992 \\
\hline & 0.0809 & 1.7600 & $5-35$ & 39 & 0.850 & 0.331 & $\ln$ & 1.056 & c.poor & British Columbia & Feller, 1992 \\
\hline & 0.0456 & 1.7009 & $2-162$ & 123 & 0.860 & 0.695 & $\ln$ & 1.275 & comp & Oregon, Washington & Gholz et al., 1979 \\
\hline & 0.0423 & 1.8619 & $5-54$ & 60 & 0.863 & 0.482 & $\ln$ & 1.123 & $n / a$ & British Columbia & Marshall and Wang, $1995^{\circ}$ \\
\hline \multirow[t]{7}{*}{ BR: } & 0.2624 & 1.5464 & $1-86$ & 22 & 0.927 & 0.661 & $\ln$ & 1.244 & comp & Idaho, Montana & Brown, $1978^{b}$ \\
\hline & 0.2308 & 1.5660 & $5-56$ & 10 & 0.960 & 0.288 & $\ln$ & 1.042 & good & British Columbia & Feller, 1992 \\
\hline & 0.0525 & 1.9040 & $6-29$ & 8 & 0.780 & 0.590 & $\ln$ & 1.190 & poor & British Columbia & Feller, 1992 \\
\hline & 0.0591 & 1.9370 & $5-64$ & 46 & 0.830 & 0.504 & $\ln$ & 1.135 & c.good & British Columbia & Feller, 1992 \\
\hline & 0.0543 & 1.9700 & $5-35$ & 42 & 0.760 & 0.500 & $\ln$ & 1.133 & c.poor & British Columbia & Feller, 1992 \\
\hline & 0.0204 & 2.1382 & $2-162$ & 123 & 0.920 & 0.695 & $\ln$ & 1.273 & comp & Oregon, Washington & Gholz et al., 1979 \\
\hline & 0.0088 & 2.5840 & $5-54$ & 60 & 0.910 & 0.528 & $\ln$ & 1.149 & $\mathrm{n} / \mathrm{a}$ & British Columbia & Marshall and Wang, $1995^{\mathrm{b}}$ \\
\hline
\end{tabular}


Fir, grand ( Abies grandis (Dougl.) Lindl.)

$\begin{array}{lllllllllll}\text { ST: } & 0.2107 & 1.6149 & 1-11 & 24 & 0.923 & 0.374 & \text { In } & 1.072 & \text { comp } & \text { Idaho, Montana } \\ \text { FL: } & 0.2923 & 1.4177 & 1-40 & 20 & 0.943 & 0.405 & \text { In } & 1.085 & \text { comp } & \text { Idaho, Montana } \\ \text { BR: } & 0.1516 & 1.6481 & 1-40 & 20 & 0.936 & 0.500 & \text { ln } & 1.133 & \text { comp } & \text { Idaho, Montana }\end{array}$

Brown, 1978 Brown, 1978 Brown, 1978

Fir, noble (Abies procera Rehd.)

$\begin{array}{lllllllllll}\text { SW: } & 0.0236 & 2.7592 & 19-111 & 6 & 0.990 & 0.251 & \ln & 1.032 & \mathrm{n} / \mathrm{a} & \text { Oregon } \\ \text { SB: } & 0.0022 & 2.8943 & 19-111 & 6 & 0.990 & 0.243 & \mathrm{ln} & 1.030 & \mathrm{n} / \mathrm{a} & \text { Oregon } \\ \text { FL: } & 0.0075 & 2.1683 & 19-111 & 6 & 0.990 & 0.184 & \ln & 1.017 & \mathrm{n} / \mathrm{a} & \text { Oregon } \\ \text { BR: } & 0.0138 & 2.3324 & 19-111 & 6 & 0.940 & 0.446 & \mathrm{ln} & 1.105 & \mathrm{n} / \mathrm{a} & \text { Oregon }\end{array}$

Fir, Pacific silver (Abies amabilis (Dougl.) Forbes)

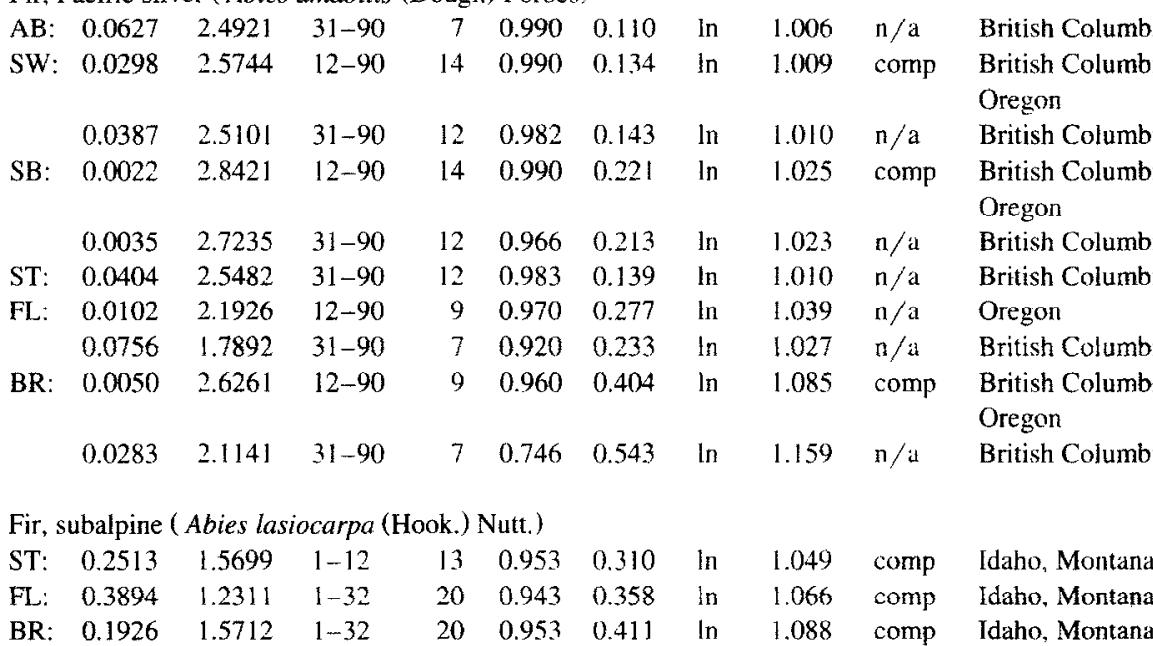

Hemlock, eastern (Tsuga canadensis (L.) Carr.)

\begin{tabular}{|c|c|c|c|c|c|c|c|c|c|c|}
\hline \multirow[t]{3}{*}{$\mathrm{AB}:$} & 0.0622 & 2.4500 & $5-50$ & 21 & 0.960 & $n / a$ & $\log$ & $\mathrm{n} / \mathrm{a}$ & $\mathrm{n} / \mathrm{a}$ & West Virginia \\
\hline & 0.1617 & 2.1536 & $2-34$ & 49 & 0.987 & 0.199 & $\ln$ & 1.020 & $\mathrm{n} / \mathrm{a}$ & New Brunswick \\
\hline & 0.0991 & 2.3617 & $3-51$ & 36 & $\mathrm{n} / \mathrm{a}$ & $n / a$ & calc & $\mathrm{n} / \mathrm{a}$ & comp & Maine \\
\hline SW: & 0.0545 & 2.3570 & $2-34$ & 49 & 0.990 & 0.141 & In & 1.010 & $\mathrm{n} / \mathrm{a}$ & New Brunswick \\
\hline SB: & 0.0138 & 2.2660 & $2-34$ & 49 & 0.985 & 0.243 & $\ln$ & 1.030 & $n / a$ & New Brunswick \\
\hline \multirow[t]{3}{*}{ ST: } & 0.1110 & 2.1400 & $1-24$ & 4 & 0.995 & 0.263 & In & 1.035 & comp & New Hampshire \\
\hline & 0.0682 & 2.3418 & $2-34$ & 49 & 0.990 & 0.141 & $\ln$ & 1.010 & $n / a$ & New Brunswick \\
\hline & 0.0649 & 2.3662 & $3-51$ & 36 & 0.983 & $\mathrm{n} / \mathrm{a}$ & In & $\mathrm{n} / \mathrm{a}$ & comp & Maine \\
\hline \multirow[t]{3}{*}{ FL: } & 0.1252 & 1.5400 & $1-24$ & 4 & 0.994 & 0.221 & $\ln$ & 1.025 & comp & New Harnpshire \\
\hline & 0.0454 & 1.6829 & $2-34$ & 49 & 0.928 & 0.280 & $\ln$ & 1.040 & $\mathrm{n} / \mathrm{a}$ & New Brunswick \\
\hline & 0.0369 & 2.0300 & $3-51^{a}$ & 36 & 0.937 & $n / a$ & $\ln$ & $n / a$ & comp & Maine \\
\hline \multirow[t]{3}{*}{ BR: } & 0.0848 & 1.9000 & $1-24$ & 4 & 0.998 & 0.163 & $\ln$ & 1.013 & comp & New Hampshire \\
\hline & 0.0586 & 1.9157 & $2-34$ & 49 & 0.927 & 0.312 & $\ln$ & 1.050 & $\mathrm{n} / \mathrm{a}$ & New Brunswick \\
\hline & 0.0062 & 2.7033 & $3-51^{\text {a }}$ & 36 & 0.960 & $n / a$ & $\ln$ & $\mathrm{n} / \mathrm{a}$ & comp & Maine \\
\hline
\end{tabular}

Hemlock, mountain (Tsuga mertensiana (Bong.) Carr.)

\begin{tabular}{|c|c|c|c|c|c|c|c|c|c|}
\hline 0.5038 & 2.0154 & $44-76$ & 5 & 0.987 & 0.061 & $\ln$ & 1.002 & $n / a$ & British Columbia \\
\hline 0.0079 & 2.9308 & $17-76$ & 14 & 0.980 & 0.228 & $\ln$ & 1.026 & comp & $\begin{array}{l}\text { British Columbia. } \\
\text { Oregon }\end{array}$ \\
\hline 0.1190 & 2.2709 & $32-76$ & 8 & 0.984 & 0.094 & In & 1.004 & $\mathrm{n} / \mathrm{a}$ & British Columbia \\
\hline 0.0037 & 2.7654 & $17-76$ & 14 & 0.970 & 0.226 & $\ln$ & 1.026 & comp & $\begin{array}{l}\text { British Columbia, } \\
\text { Oregon }\end{array}$ \\
\hline 0.0157 & 2.4258 & $32-76$ & 8 & 0.965 & 0.153 & $\ln$ & 1.012 & $n / a$ & British Columbia \\
\hline 0.1314 & $? .3011$ & $32-76$ & 8 & 0.985 & 0.093 & $\ln$ & 1.004 & $n / a$ & British Columhia \\
\hline 0.0022 & 1.9756 & $17-76$ & 11 & 0.970 & 0.158 & In & 1.013 & comp & $\begin{array}{l}\text { British Columbia. } \\
\text { Oregon }\end{array}$ \\
\hline
\end{tabular}

\author{
Gholz et al.. 1979 \\ Gholz et al.. 1979 \\ Gholz et al. 1979 \\ Gholz et al.. 1970
}

Krumlik, $1974^{\mathrm{i}}$

Gholz et al. 1979

Krumlik, $1974^{\text {b }}$

Gholz et al.. 1979

Krumlik, $1974^{\circ}$

Krumlik. $1974^{\text {th }}$

Ghols el al. 1979

Krumlik. $1974^{\text {h }}$

Gholz et al., 1979

Krumlik, $1974^{k}$

Brown. $1978^{\text {r }}$

Brown, 1978 .

Brown. $1978^{\text {h }}$

Brenneman et al.. 1978

Ker. 1980 a

Young et al., 1980

Ker, 1980a

Ker. 1980a

Hocker and Earley, 1983

Ker. 1980a

Young et al, 1980

Hocker and Earley, 1983

Ker, 1980a

Young et al., 1980

Hocker and Earley, 1983

Ker. 1980a

Young et al. 1980

Krumlik, 1974 ${ }^{\text {t }}$

Gholz et al.. 1979

Krumlik, $1974^{\circ}$

Gholz et al. 1979

Krumlik, $1974^{\circ}$

Knumlik, 1974 b

Gholz et al., 1979 


\begin{tabular}{|c|c|c|c|c|c|c|c|c|c|c|c|}
\hline \multirow{3}{*}{ BR: } & 0.0089 & 2.4701 & $44-76$ & 5 & 0.994 & 0.051 & $\ln$ & 1.001 & $\mathrm{n} / \mathrm{a}$ & British Columbia & Krumlik, $1974^{\text {b }}$ \\
\hline & 0.0052 & 2.6045 & $17-76$ & 11 & 0.990 & 0.122 & $\ln$ & 1.006 & comp & $\begin{array}{l}\text { British Columbia, } \\
\text { Oregon }\end{array}$ & Gholz et al., 1979 \\
\hline & 0.1575 & 1.4855 & $44-76$ & 5 & 0.803 & 0.191 & $\ln$ & 1.018 & $\mathrm{n} / \mathrm{a}$ & British Columbia & Krumlik, $1974^{\text {b }}$ \\
\hline \multicolumn{12}{|c|}{ Hemlock, western (Tsuga heterophylla (Raf.) Sarg.) } \\
\hline AB: & 0.2570 & 2.1349 & $16-49$ & 8 & 0.967 & 0.171 & ln & .015 & $\mathrm{n} / \mathrm{a}$ & British Col & Krumlik, $1974^{\text {b }}$ \\
\hline \multirow[t]{2}{*}{ SW: } & 0.1132 & 2.2570 & $15-78$ & 18 & 0.990 & 0.118 & $\ln$ & .007 & comp & $\begin{array}{l}\text { British Columbia. } \\
\text { Oregon }\end{array}$ & et al., 1979 \\
\hline & 0.1638 & 2.1202 & $16-49$ & 8 & 0.983 & 0.121 & in & 1.007 & $\mathrm{n} / \mathrm{a}$ & British Columbia & Krumlik, $1974^{\circ}$ \\
\hline \multirow[t]{2}{*}{ SB: } & 0.0125 & 2.2580 & $15-78$ & 18 & 0.990 & 0.138 & $\ln$ & 1.010 & comp & $\begin{array}{l}\text { British Columbia, } \\
\text { Oregon }\end{array}$ & Gholz et al.. 1979 \\
\hline & 0.0140 & 2.3940 & $16-49$ & 8 & 0.937 & 0.271 & $\ln$ & 1.037 & $\mathrm{n} / \mathrm{a}$ & Columbia & $974^{b}$ \\
\hline \multirow[t]{2}{*}{ ST: } & 0.4208 & 1.2501 & $0-13$ & 13 & 0.922 & 0.434 & $\ln$ & 1.099 & comp & Idaho, Mon & Brown, $1978^{b}$ \\
\hline & 0.1695 & 2.1687 & $16-49$ & 8 & 0.979 & 0.139 & $\ln$ & 1.010 & $\mathrm{n} / \mathrm{a}$ & British Columbia & Krumlik, $1974^{b}$ \\
\hline \multirow[t]{3}{*}{ FL: } & 0.3203 & 1.1343 & $0-18$ & 16 & 0.909 & 0.454 & $\ln$ & 1.108 & comp & Idaho, Montana & Brown, $1978^{b}$ \\
\hline & 0.0146 & 2.1280 & $15-78$ & 18 & 0.960 & 0.435 & $\ln$ & 1.099 & comp & $\begin{array}{l}\text { British Columbia, } \\
\text { Oregon }\end{array}$ & Gholz et al.. 1979 \\
\hline & 0.0040 & 2.4543 & $16-49$ & 0 & 0.890 & 0.378 & $\ln$ & 1.074 & $\mathrm{n} / \mathrm{a}$ & British Columbia & $k, 1974^{b}$ \\
\hline \multirow[t]{3}{*}{ BR: } & 0.2392 & 1.3060 & $0-18$ & 16 & 0.919 & 0.492 & $\ln$ & 1.129 & comp & Idaho, Montana & Brown, $1978^{b}$ \\
\hline & 0.0053 & 2.7780 & $15-78$ & 18 & 0.980 & 0.421 & $\ln$ & 1.093 & comp & $\begin{array}{l}\text { British Columbia, } \\
\text { Oregon }\end{array}$ & Gholl et al., 1979 \\
\hline & 0.0948 & 1.9641 & $16-49$ & 8 & 0.848 & 0.363 & $n$ & 1.068 & $n / a$ & British Columbia & Krumlik, $1974^{\text {h }}$ \\
\hline
\end{tabular}

Hickory, all ( Carva spp.)

\begin{tabular}{|c|c|c|c|c|c|c|c|c|c|}
\hline AB: & 0.0792 & 2.6349 & $5-50$ & 14 & 0.990 & $\mathrm{n} / \mathrm{a}$ & $\log$ & $\mathrm{n} / \mathrm{a}$ & West Virginia \\
\hline & 0.0763 & 2.6209 & $5-40^{a}$ & 19 & 0.987 & 41.28 & abs & $\mathrm{n} / \mathrm{a}$ & $22.3(50) \mathrm{W}$ \\
\hline & 0.0043 & 3.3743 & $5-40^{2}$ & 19 & 0.970 & 45.85 & abs & $\mathrm{n} / \mathrm{a}$ & . \\
\hline & 0.0066 & 3.2817 & $5-40^{a}$ & 19 & 0.972 & 48.12 & abs & $\mathrm{n} / \mathrm{a}$ & 22.3(50) West Virgi \\
\hline
\end{tabular}

Brenneman et al.. 1978

Wiant et al. $1977^{d}$

Wiant et al., 1977

Wiant ct al.. 1977

Juniper, western (Juniperus occidentalis Hooker)

$\begin{array}{lllllllllll}\text { SW: } & 0.0002 & 2.6389 & 15-273 & 10 & 0.990 & 0.170 & \ln & 1.015 & \mathrm{n} / \mathrm{a} & \text { Oregon } \\ \text { SB: } & 0.00004 & 2.6333 & 15-273 & 10 & 0.990 & 0.390 & \ln & 1.079 & \mathrm{n} / \mathrm{a} & \text { Oregon } \\ \text { FL: } & 0.0144 & 1.5606 & 15-273 & 10 & 0.990 & 0.155 & \mathrm{ln} & 1.012 & \mathrm{n} / \mathrm{a} & \text { Oregon } \\ \text { BR: } & 0.0007 & 2.3337 & 15-273 & 10 & 0.990 & 0.261 & \ln & 1.035 & \mathrm{n} / \mathrm{a} & \text { Oregon }\end{array}$

Larch, eastern (tamarack) (Larix laricina (Du Roi) Koch.)

$\begin{array}{lllllllllll}\text { AB: } & 0.1359 & 2.2980 & 7-30 & 53 & 0.978 & 0.058 & \log & 1.004 & \mathrm{n} / \mathrm{a} & \text { Minnesota } \\ & 0.0946 & 2.3572 & 2-31 & 47 & 0.990 & 0.141 & \ln & 1.010 & \mathrm{n} / \mathrm{a} & \text { Nova Scotia } \\ & 0.1265 & 2.2453 & 3-51^{\mathrm{a}} & 23 & \mathrm{n} / \mathrm{a} & \mathrm{n} / \mathrm{a} & \mathrm{calc} & \mathrm{n} / \mathrm{a} & \mathrm{comp} & \text { Maine } \\ \mathrm{SW}: & 0.0731 & 2.3930 & 7-30 & 53 & 0.963 & 0.079 & \log & 1.007 & \mathrm{n} / \mathrm{a} & \text { Minnesota } \\ & 0.0464 & 2.5050 & 2-31 & 47 & 0.980 & 0.141 & \ln & 1.010 & \mathrm{n} / \mathrm{a} & \text { Nova Scotia } \\ \mathrm{SB}: & 0.0168 & 2.0868 & 2-31 & 47 & 0.990 & 0.141 & \ln & 1.010 & \mathrm{n} / \mathrm{a} & \text { Nova Scotia } \\ \mathrm{ST}: & 0.0609 & 2.4472 & 2-31 & 47 & 0.980 & 0.141 & \ln & 1.010 & \mathrm{n} / \mathrm{a} & \text { Nova Scotia } \\ & 0.0762 & 2.3051 & 3-51^{\mathrm{a}} & 23 & 0.995 & \mathrm{n} / \mathrm{a} & \mathrm{ln} & \mathrm{n} / \mathrm{a} & \mathrm{comp} & \text { Maine } \\ \mathrm{FL}: & 0.0061 & 1.9790 & 2-31^{a} & 47 & 0.770 & 0.457 & \ln & 1.110 & \mathrm{n} / \mathrm{a} & \text { Nova Scotia } \\ & 0.0466 & 1.7250 & 3-51^{\mathrm{a}} & 23 & 0.954 & \mathrm{n} / \mathrm{a} & \ln & \mathrm{n} / \mathrm{a} & \mathrm{comp} & \text { Maine } \\ \mathrm{BR}: & 0.0776 & 2.0550 & 7-30 & 53 & 0.804 & 0.172 & \log & 1.035 & \mathrm{n} / \mathrm{a} & \text { Minnesota } \\ & 0.0178 & 2.1727 & 2-31^{a} & 47 & 0.800 & 0.341 & \ln & 1.060 & \mathrm{n} / \mathrm{a} & \text { Nova Scotia } \\ & 0.0436 & 1.9810 & 3-51^{\mathrm{a}} & 23 & 0.960 & \mathrm{n} / \mathrm{a} & \ln & \mathrm{n} / \mathrm{a} & \mathrm{comp} & \text { Maine }\end{array}$

Larch, western ( Larix occidentalis Nutt.)

$\begin{array}{lllllllllll}\text { ST: } & 0.2942 & 1.5593 & 1-12 & 13 & 0.907 & 0.502 & \text { ln } & 1.134 & \text { comp } & \text { Idaho, Montana } \\ \text { FL: } & 0.1307 & 1.0557 & 1-17 & 14 & 0.923 & 0.324 & \text { ln } & 1.054 & \text { comp } & \text { Idaho, Montana } \\ \text { BR: } & 0.1821 & 1.2885 & 1-17 & 14 & 0.900 & 0.459 & \text { In } & 1.111 & \text { comp } & \text { Idaho, Montana }\end{array}$

Gholz et al., 1979

Gholz et al.. 1979

Gholz et al., 1979

Gholz et al., 1979

Carpenter, 1983

Ker, 1980b

Young et al., 1980

Carpenter, 1983

Ker, 1980b

Ker, 1980b

Ker, $1980 b$

Young et al., 1980

Ker, 1980b

Young et al., 1980

Carpenter, 1983

Ker, 1980b

Young et al., 1980

Brown, $1978^{b}$

Brown, $1978^{b}$

Brown, $1978^{b}$ 
Madrone, Pacific ( Arbutus menziesii Pursh)

\begin{tabular}{|c|c|c|c|c|c|c|c|c|c|c|c|}
\hline FL: & 0.0381 & 1.5274 & $3-63$ & 31 & 0.835 & 0.558 & In & 1.168 & comp & Oregon, California & Snell and Little. $1983^{\circ}$ \\
\hline BR: & 0.0093 & 2.6868 & $3-63$ & 31 & 0.886 & 0.794 & $\ln$ & 1.371 & comp & Oregon. California & Snell and Little. $1983^{h}$ \\
\hline
\end{tabular}

Maple, bigleat ( Acer macrophyllum Pursh)

$\begin{array}{llllllllllll}\text { SW: } & 0.0302 & 2.7230 & 8-35 & 18 & 0.990 & 0.118 & \text { In } & 1.007 & \text { comp } & \text { Oregon } & \text { Gholz et al.. 1979 } \\ \text { SB: } & 0.0100 & 2.5740 & 8-35 & 18 & 0.980 & 0.241 & \text { ln } & 1.029 & \text { comp } & \text { Oregon } & \text { Gholz et al.. 1979 } \\ \text { FL: } & 0.0220 & 1.6170 & 8-35 & 18 & 0.870 & 0.318 & \text { In } & 1.052 & \text { comp } & \text { Oregon } & \text { Gholz et al., 1979. } \\ & 0.0324 & 1.3979 & 4-44 & 16 & 0.887 & 0.317 & \text { In } & 1.052 & \text { comp } & \text { Washington } & \text { Snell and Little. 1983 b } \\ \text { BR: } & 0.0129 & 2.4300 & 8-35 & 18 & 0.880 & 0.474 & \text { in } & 1.119 & \text { comp } & \text { Oregon } & \text { Gholz et al., } 1979 \\ & 0.0195 & 2.4204 & 4-44 & 16 & 0.887 & 0.548 & \text { ln } & 1.162 & \text { comp } & \text { Washington } & \text { Snell and Little. 1983 }\end{array}$

Maple, mountain ( Acer spicatum Lamb.)

$\begin{array}{llllrllllll}\text { AB: } & 0.2040 & 2.2524 & 1-20^{a} & 15 & 0.990 & 0.074 & \log & 1.006 & \text { comp } & \text { New Hampshire } \\ \text { SW: } & 0.0754 & 2.3364 & 1-20^{a} & 15 & 0.994 & 0.064 & \log & 1.005 & \text { comp } & \text { New Hampshire } \\ \text { SB: } & 0.0177 & 2.1133 & 1-20^{a} & 15 & 0.988 & 0.078 & \log & 1.007 & \text { comp } & \text { New Hampshire } \\ \text { ST: } & 0.0767 & 2.9400 & 1-3 & 6 & 0.991 & 0.163 & \ln & 1.013 & \text { comp } & \text { New Hampshire } \\ & 0.0926 & 2.3040 & 1-20^{a} & 15 & 0.994 & 0.063 & \log & 1.005 & \text { comp } & \text { New Hampshire } \\ \text { BR: } & 0.0184 & 2.2400 & 1-3 & 6 & 0.957 & 0.403 & \ln & 1.085 & \text { comp } & \text { New Hampshire } \\ & 0.0079 & 3.1640 & 1-20^{a} & 15 & 0.964 & 0.200 & \log & 1.047 & \text { comp } & \text { New Hampshire }\end{array}$

Whittaker et al., 1974 Whittaker et al.. 1974 Whittaker et al.. 1974 Hocker and Earley, 1983 Whittaker et al., 1974 Hocker and Earley. 1983 Whittaker et al.. 1974

Maple, red ( Acer nubrum L.)

\begin{tabular}{|c|c|c|c|c|c|c|c|c|c|c|c|}
\hline \multirow[t]{10}{*}{$\mathrm{AB}$ : } & 0.0910 & 2.5080 & $5-50$ & 27 & 0.990 & $\mathrm{n} / \mathrm{a}$ & $\log$ & $\mathrm{n} / \mathrm{a}$ & $\mathrm{n} / \mathrm{a}$ & West Virginia & Brenneman et al, 1978 \\
\hline & 0.1651 & 2.2394 & $8-26$ & 22 & 0.965 & 0.152 & $\ln$ & 1.012 & comp & Rhode Island & Bridge, $1979^{\circ}$ \\
\hline & 0.1789 & 2.3340 & $10-52$ & 150 & 0.980 & 0.116 & $\ln$ & 1.007 & comp & Michigan, Wisconsin & Crow and Erdmann, 1983 \\
\hline & 0.1394 & 2.3405 & $1-31$ & 37 & 0.992 & 0.176 & $\ln$ & 1.016 & $\mathrm{n} / \mathrm{a}$ & Nova Scotia & Freedman et al., 1982 \\
\hline & 0.1317 & 3199 & $1-30$ & 49 & 0.990 & 0.141 & In & 1.010 & $\mathrm{n} / \mathrm{a}$ & Nova Scotia & Ker, 1980b \\
\hline & 0.1970 & 2.1933 & $0-35$ & 198 & 0.965 & 34.00 & abs & $\mathrm{n} / \mathrm{a}$ & comp & $\begin{array}{l}\text { New Brunswick, } \\
\text { Nova Scotia }\end{array}$ & Ker, 1984 \\
\hline & 0.2582 & .6728 & $0-10$ & 30 & 0.877 & $\mathrm{n} / \mathrm{a}$ & $\log$ & $\mathrm{n} / \mathrm{a}$ & $n / a$ & New Brunswick & MacLean and Wein, 1976 \\
\hline & 0.1618 & 2.3095 & $4-35$ & 45 & $n / a$ & $\mathrm{n} / \mathrm{a}$ & calc & $n / a$ & $\mathrm{n} / \mathrm{a}$ & Upper Great Lakes & Perala and Alban, 1994 \\
\hline & 0.0755 & 2.5623 & $5-40 "$ & 19 & 0.981 & 35.38 & bs & $n / a$ & $22.3(50)$ & West Virginia & Wiant et al., 1977 \\
\hline & 0.1262 & 2.3804 & $3-66^{a}$ & 62 & $n / a$ & $n / a$ & calc & $n / a$ & comp & Maine & Young et al., 1980 \\
\hline \multirow[t]{5}{*}{ SW: } & 0.1599 & 2.2340 & $10-52$ & 150 & 0.970 & 0.152 & $\mathrm{n}$ & 1.012 & comp & Michigan, Wisconsin & Crow and Erdmann, 1983 \\
\hline & 0.0394 & 2.6242 & $1-31$ & 26 & 0.960 & 0.196 & $\mathrm{n}$ & 1.019 & $n / a$ & Scotia & net al. 1982 \\
\hline & 0.0783 & 2.3795 & $1-30$ & 49 & 0.980 & 0.199 & & 1.020 & $n / a$ & Scotia & Ker \\
\hline & 0.1139 & 2.2342 & $0-35$ & 198 & 0.961 & 23.90 & bs & $\mathrm{n} / a$ & comp & $\begin{array}{l}\text { Brunswick. } \\
\text { Scotia }\end{array}$ & Ker. \\
\hline & 0.0969 & 2.3398 & $4-35$ & 45 & $\mathrm{n} / \mathrm{a}$ & $\mathrm{n} / \mathrm{a}$ & calc & $\mathrm{n} / \mathrm{a}$ & $\mathrm{n} / \mathrm{a}$ & Upper Great Lakes & 994 \\
\hline \multirow[t]{4}{*}{ SB: } & 0.0405 & 1.9850 & $10-52$ & 150 & 0.950 & 0.186 & $\ln$ & 1.017 & corinp & Michigan, Wisconsin & Erdmanu, 1983 \\
\hline & 0.0113 & 2.3717 & $1-31$ & 26 & 0.945 & 0.208 & $\ln$ & 1.022 & $\mathrm{n} / \mathrm{a}$ & Nova Scotia & et.al. 1982 \\
\hline & 0.0219 & 2.1419 & $1-30$ & 50 & 0.970 & 0.243 & $\ln$ & 1.030 & $n / a$ & No & \\
\hline & 0.0210 & 2.1910 & $4-35$ & 44 & 0.976 & $\mathrm{n} / \mathrm{a}$ & $\pi$ & $\mathrm{n} / \mathrm{a}$ & $n / a$ & Upper Great Lakes & d Alban, 1994 \\
\hline \multirow[t]{9}{*}{ ST: } & 0.0491 & 2.5932 & $1-31$ & 26 & 0.960 & 0.194 & $\ln$ & 1.019 & $\mathrm{n} / \mathrm{a}$ & Scotia & t al.. 1982 \\
\hline & 0.1290 & 2.3300 & $0-27$ & 33 & 0.992 & 0.222 & ln & 1.025 & comp & New Hampshire & nd Earley, 1983 \\
\hline & 0.0996 & 2.3418 & $1-30$ & 49 & 0.980 & 0.199 & $\ln$ & 1.020 & $\mathrm{n} / \mathrm{a}$ & Nova Scotia & Ker. 1980b \\
\hline & 0.1351 & 2.2215 & $0-35$ & 198 & 0.962 & 26.90 & $a b s$ & $\mathrm{n} / \mathrm{a}$ & comp & $\begin{array}{l}\text { New Brunswick, } \\
\text { Nova Scotia }\end{array}$ & $\mathrm{Ke}$ \\
\hline & 0.1285 & 2.2940 & $3-12$ & 7 & 0.891 & 0.421 & $\ln$ & 1.093 & $\mathrm{n} / \mathrm{a}$ & New Hampshire & d \\
\hline & 0.1380 & 1.7963 & $0-10$ & 30 & 0.861 & $\mathrm{n} / \mathrm{a}$ & $\log$ & $\mathrm{n} / \mathrm{a}$ & $\mathrm{n} / \mathrm{a}$ & New Brunswick & MacLean and Wein, 1976 \\
\hline & 0.5788 & 2.3151 & $7-24$ & $\mathrm{n} / \mathrm{a}$ & 0.976 & $\mathrm{n} / \mathrm{a}$ & $\log$ & $\mathrm{n} / \mathrm{a}$ & $\mathrm{n} / \mathrm{a}$ & New Jersey & Reynolds et ál, 1978 \\
\hline & 0.0595 & 2.6522 & $3-15$ & 30 & 0.993 & $\mathrm{n} / \mathrm{a}$ & $\log$ & $\mathrm{n} / \mathrm{a}$ & comp & Maine & 1973 \\
\hline & 0.0787 & 2.4898 & $3-66^{a t}$ & 62 & 0.996 & $\mathrm{n} / \mathrm{a}$ & $\ln$ & $n / a$ & comp & Maine & Young et al, 1980 \\
\hline \multirow[t]{4}{*}{ FL: } & 0.0373 & 1.5400 & $10-52$ & 150 & 0.540 & 0.569 & $\ln$ & 1.173 & comp & Michigan, Wisconsin & Crow and Erdmann, 1983 \\
\hline & 0.0261 & 1.5914 & $1-31$ & 37 & 0.908 & 0.420 & $\ln$ & 1.092 & $\mathrm{n} / \mathrm{a}$ & Nova Scotia & Freedman et al. 1982 \\
\hline & 0.0153 & 1.9300 & $0-27$ & 9 & 0.989 & 0.274 & $\ln$ & 1.038 & comp & New Hampshire & Hocker and Earley, 1983 \\
\hline & 0.0174 & 1.6529 & $1-30$ & 50 & 0.920 & 0.280 & $\ln$ & 1.040 & $n / a$ & Nova Scotia & Ker, $1980 \mathrm{~b}$ \\
\hline
\end{tabular}




\begin{tabular}{|c|c|c|c|c|c|c|c|c|c|c|}
\hline 0.0408 & 1.5518 & $0-35$ & 197 & 0.879 & 1.600 & abs & $\mathrm{n} / \mathrm{a}$ & comp & $\begin{array}{l}\text { New Brunswick. } \\
\text { Nova Scotia }\end{array}$ & Ker, 1984 \\
\hline 0.0018 & 2.7770 & $3-12$ & 7 & 0.998 & 0.061 & In & 1.002 & $\mathrm{n} / \mathrm{a}$ & New Hampshire & $\begin{array}{l}\text { Kinerson and } \\
\text { Bartholomew, } 1977\end{array}$ \\
\hline 0.0191 & 1.8670 & $4-35$ & 45 & 0.820 & $\mathrm{n} / \mathrm{a}$ & $\ln$ & $\mathrm{n} / \mathrm{a}$ & $\mathrm{n} / \mathrm{a}$ & Upper Great Lakes & Perala and Alban, 1994 \\
\hline 1.6959 & 1.3100 & $7-24$ & $\mathrm{n} / \mathrm{a}$ & 0.582 & $\mathrm{n} / \mathrm{a}$ & $\log$ & $\mathrm{n} / \mathrm{a}$ & $\mathrm{n} / \mathrm{a}$ & New Jersey & Reynolds et al., 1978 \\
\hline 0.0248 & 1.8015 & $3-15$ & 30 & 0.852 & $n / a$ & $\log$ & $\mathrm{n} / \mathrm{a}$ & $\operatorname{comp}$ & Maine & Ribe, 1973 \\
\hline 0.0249 & 1.8322 & $3-66^{a}$ & 62 & 0.953 & $\mathrm{n} / \mathrm{a}$ & $\ln$ & $\mathrm{n} / \mathrm{a}$ & comp & Maine & Young et al., 1980 \\
\hline $\mathrm{R}: \quad 0.0075$ & 2.8310 & $10-52$ & 150 & 0.850 & 0.479 & $\ln$ & 1.122 & comp & Michigan, Wisconsin & Crow and Erdmann, 1983 \\
\hline 0.3295 & 1.5219 & $1 \cdot 31$ & 37 & 0.916 & 0.381 & $\ln$ & 1.075 & $\mathrm{n} / \mathrm{a}$ & Nova Scotia & Freedman et al., 1982 \\
\hline 0.0182 & 2.4100 & $0-27$ & 33 & 0.952 & 0.614 & $\ln$ & 1.207 & comp & New Hampshire & Hocker and Earley, 1983 \\
\hline 0.0180 & 2.3506 & $1-30$ & 49 & 0.940 & 0.341 & $\ln$ & 1.060 & $\mathrm{n} / \mathrm{a}$ & Nova Scotia & Ker, $1980 \mathrm{~b}$ \\
\hline 0.0634 & 2.0709 & $0-35$ & 197 & 0.868 & 14.80 & abs & $\mathrm{n} / \mathrm{a}$ & comp & $\begin{array}{l}\text { New Brunswick, } \\
\text { Nova Scotia }\end{array}$ & Ker, 1984 \\
\hline 0.0181 & 1.9600 & $3-12$ & 7 & 0.845 & 0.443 & $\ln$ & 1.103 & $\mathrm{n} / \mathrm{a}$ & New Hampshire & $\begin{array}{l}\text { Kinerson and } \\
\text { Bartholomew, } 1977\end{array}$ \\
\hline 0.0113 & 1.8989 & $7-24$ & $\mathrm{n} / \mathrm{a}$ & 0.906 & $\mathrm{n} / \mathrm{a}$ & $\log$ & $\mathrm{n} / \mathrm{a}$ & $\mathrm{n} / \mathrm{a}$ & New Jersey & Reynolds et al., 1978 \\
\hline 0.0342 & 1.9148 & $3-15$ & 30 & 0.896 & $\mathrm{n} / \mathrm{a}$ & $\log$ & $\mathrm{n} / \mathrm{a}$ & comp & Maine & Ribe, 1973 \\
\hline 0.0223 & 2.2055 & $3-66^{a}$ & 62 & 0.966 & $\mathrm{n} / \mathrm{a}$ & $\ln$ & $\mathrm{n} / \mathrm{a}$ & comp & Maine & Young et al.. 1980 \\
\hline
\end{tabular}

Maple, striped ( Acer pensylvanicum L.)

\begin{tabular}{|c|c|c|c|c|c|c|c|c|c|c|c|}
\hline \\
\hline & $\begin{array}{l}0.0839 \\
0.0218\end{array}$ & $\begin{array}{l}2.3200 \\
2.5700\end{array}$ & $\begin{array}{l}1-8 \\
1-8\end{array}$ & 8 & $\begin{array}{l}0.901 \\
0.994\end{array}$ & 0.210 & $\ln$ & $\begin{array}{l}1.040 \\
1.022\end{array}$ & $\begin{array}{l}\text { comp } \\
\text { comp }\end{array}$ & $\begin{array}{l}\text { New Hampsnire } \\
\text { New Hampshire }\end{array}$ & $\begin{array}{l}\text { Hocker and Earley, } 1983 \\
\text { Hocker and Earley, } 1983\end{array}$ \\
\hline \multicolumn{12}{|c|}{ Maple, sugar ( Acer saccharum Marsh.) } \\
\hline \multirow[t]{9}{*}{$\mathrm{AB}:$} & 0.2064 & 2.3300 & $2-40$ & 5 & 0.998 & $\mathrm{n} / \mathrm{a}$ & $\ln$ & $\mathrm{n} / \mathrm{a}$ & $22(45)$ & New York & Bickelhaupt et al., $1973^{\mathrm{g}}$ \\
\hline & 0.1252 & 2.4800 & $2-40$ & 5 & 0.984 & $n / a$ & $\ln$ & $n / a$ & $22(45)$ & New York & Bickelhaupt et al., $1973^{\mathrm{h}}$ \\
\hline & 0.1008 & 2.5735 & $5-50$ & 119 & 0.980 & $\mathrm{n} / \mathrm{a}$ & $\log$ & $\mathrm{n} / \mathrm{a}$ & $\mathrm{n} / \mathrm{a}$ & West Virginia & Brenneman et al., 1978 \\
\hline & 0.1532 & 2.3924 & $1-34$ & 36 & 0.995 & 0.149 & $\ln$ & 1.011 & $\mathrm{n} / \mathrm{a}$ & Nova Scotia & Freedman et al., 1982 \\
\hline & 0.1599 & 2.3376 & $1-41$ & 45 & 0.993 & $0.14 \mathrm{~L}$ & $\ln$ & 1.010 & $n / a$ & New Brunswick & Ker, 1980a \\
\hline & 0.1259 & 2.5200 & $8-24$ & 9 & 0.990 & 0.045 & $\log$ & 1.002 & $\mathrm{n} / \mathrm{a}$ & Wisconsin & Pastor and Bockheim, 1981 \\
\hline & 0.1676 & 2.3646 & $4-34$ & 42 & $\mathrm{n} / \mathrm{a}$ & $\mathrm{n} / \mathrm{a}$ & calc & $\mathrm{n} / \mathrm{a}$ & $\mathrm{n} / \mathrm{a}$ & Upper Great Lakes & Perala and Alban, 1994 \\
\hline & 0.1641 & 2.4209 & $1-50^{a}$ & 14 & 0.998 & 0.060 & $\log$ & 1.004 & $\mathrm{n} / \mathrm{a}$ & New Hampshire & Whittaker et al., 1974 \\
\hline & 0.1791 & 2.3329 & $3-66^{a}$ & 42 & $\mathrm{n} / \mathrm{a}$ & $\mathrm{n} / \mathrm{a}$ & calc & $\mathrm{n} / \mathrm{a}$ & comp & Maine & Young et al. 1980 \\
\hline \multirow[t]{5}{*}{ SW: } & 0.0270 & 2.8318 & $1-34$ & 26 & 0.963 & 0.217 & $\ln$ & 1.024 & $n / a$ & Nova Scotia & Freedman et al., 1982 \\
\hline & 0.1024 & 2.3869 & $1-41$ & 45 & 0.990 & 0.141 & $\ln$ & 1.010 & $\mathrm{n} / \mathrm{a}$ & Brunswick & Ker, 1980a \\
\hline & 0.0731 & 2.5630 & $8-24$ & 9 & 0.990 & 0.045 & $\log$ & 1.002 & $n / a$ & Wisconsin & Pastor and Bo \\
\hline & 0.1179 & 2.3467 & $4-34$ & 42 & $\mathrm{n} / \mathrm{a}$ & $\mathrm{n} / \mathrm{a}$ & calc & $\mathrm{n} / \mathrm{a}$ & $\mathrm{n} / \mathrm{a}$ & Upper Great Lakes & Perala and Alban, 1994 \\
\hline & 0.1039 & 2.3855 & $1-50^{a}$ & 14 & 0.996 & 0.069 & $\log$ & 1.005 & $\mathrm{n} / \mathrm{a}$ & New Hampshire & Whittaker et al., 1974 \\
\hline \multirow[t]{5}{*}{$\mathrm{SB}:$} & 0.0035 & 2.8193 & $1-34$ & 26 & 0.960 & 0.222 & $\ln$ & 1.025 & $\mathrm{n} / \mathrm{a}$ & Nova Scotia & Freedman et al., 1982 \\
\hline & 0.0206 & 2.2684 & $1-41$ & 45 & 0.976 & 0.243 & In & 1.030 & $\mathrm{n} / \mathrm{a}$ & New Brunswick & Ker, \\
\hline & 0.0218 & 2.2850 & $8-24$ & 9 & 0.984 & 0.049 & $\log$ & 1.003 & $\mathrm{n} / \mathrm{a}$ & consin & 1,1981 \\
\hline & 0.0246 & 2.2401 & $4-34$ & 42 & $n / a$ & $\mathrm{n} / \mathrm{a}$ & calc & $\mathrm{n} / \mathrm{a}$ & $\mathrm{n} / \mathrm{a}$ & Upper Great Lakes & Perala and Alban, 1994 \\
\hline & 0.0179 & 2.2838 & $1-50^{a}$ & 14 & 0.992 & 0.093 & $\log$ & 1.010 & $n / a$ & New Hampshire & Whittaker et al., 1974 \\
\hline \multirow[t]{6}{*}{ ST: } & 0.0305 & 2.8305 & $1-34$ & 26 & 0.963 & 0.216 & $\ln$ & 1.024 & $n / a$ & Nova Scotia & Freedman et al.. 1982 \\
\hline & 0.1657 & 2.2900 & $1-28$ & 27 & 0.994 & 0.188 & $\ln$ & 1.018 & comp & New Hampshire & Hocker and Earley, 1983 \\
\hline & 0.1265 & 2.3603 & $1-41$ & 45 & 0.991 & 0.141 & $\ln$ & 1.010 & $\mathrm{n} / \mathrm{a}$ & New Brunswick & Ker, 1980a \\
\hline & 0.1127 & 2.4927 & $3-15$ & 30 & 0.993 & $\mathrm{n} / \mathrm{a}$ & $\log$ & $n / a$ & comp & Maine & Ribe, 1973 \\
\hline & 0.1224 & 2.3718 & $1-50^{a}$ & 14 & 0.996 & 0.065 & $\log$ & 1.005 & $\mathrm{n} / \mathrm{a}$ & New Hampshire & Whittaker et al., 1974 \\
\hline & 0.1626 & 2.2894 & $3-66^{a}$ & 42 & 0.995 & $\mathrm{n} / \mathrm{a}$ & $\ln$ & $\mathrm{n} / \mathrm{a}$ & comp & Maine & Young et al., 1980 \\
\hline \multirow[t]{7}{*}{ FL: } & 0.0051 & 2.2200 & $2-40$ & 5 & 0.974 & $\mathrm{n} / \mathrm{a}$ & In & $\mathrm{n} / \mathrm{a}$ & $22(45)$ & New York & Bickelhaupt et al., $1973^{\mathrm{g}}$ \\
\hline & 0.0178 & 1.7600 & $2-40$ & 5 & 0.874 & $\mathrm{n} / \mathrm{a}$ & $\ln$ & $\mathrm{n} / \mathrm{a}$ & $22(45)$ & New York & Bickelhaupt et al., $1973^{\mathrm{h}}$ \\
\hline & 0.0112 & 1.9557 & $1-34$ & 36 & 0.958 & 0.354 & $\ln$ & 1.065 & $\mathrm{n} / \mathrm{a}$ & Nova Scotia & et al., 1982 \\
\hline & 0.0064 & 2.4200 & $1-28$ & 8 & 0.976 & 0.415 & $\ln$ & 1.090 & comp & New Hampshire & Hocker and Earley, 1983 \\
\hline & 0.0154 & 1.6990 & $1-41$ & 45 & 0.928 & 0.280 & $\ln$ & 1.040 & $n / a$ & New Brunswick & Ker, $1980 \mathrm{a}$ \\
\hline & 0.0230 & 1.6701 & $3-15$ & 30 & 0.827 & $\mathrm{n} / \mathrm{a}$ & $\log$ & $\mathrm{n} / \mathrm{a}$ & comp & Maine & Ribe, 1973 \\
\hline & 0.0060 & 2.2240 & $8-24$ & 9 & 0.874 & 0.149 & $\log$ & 1.026 & $\mathrm{n} / \mathrm{a}$ & Wisconsin & Pastor and Bockheim, \\
\hline
\end{tabular}




$\begin{array}{lllllllllll}0.0370 & 1.6950 & 4-34 & 40 & 0.860 & 0.405 & \ln & 1.085 & \mathrm{n} / \mathrm{a} & \text { Upper Great Lakes } \\ \mathrm{BR}: & 0.0164 & 1.8901 & 3-66^{\mathrm{a}} & 42 & 0.951 & \mathrm{n} / \mathrm{a} & \ln & \mathrm{n} / \mathrm{a} & \mathrm{comp} & \text { Maine } \\ 0.3558 & 1.5812 & 1-34 & 36 & 0.913 & 0.424 & \ln & 1.094 & \mathrm{n} / \mathrm{a} & \text { Nova Scotia } \\ 0.0297 & 2.2100 & 1-28 & 27 & 0.936 & 0.659 & \ln & 1.243 & \mathrm{comp} & \text { New Hampshire } \\ 0.0175 & 2.3841 & 1-41 & 45 & 0.908 & 0.457 & \ln & 1.110 & \mathrm{n} / \mathrm{a} & \text { New Brunswick } \\ 0.0262 & 2.5070 & 8-24 & 9 & 0.914 & 0.137 & \log & 1.022 & \mathrm{n} / \mathrm{a} & \text { Wisconsin } \\ 0.0589 & 1.5571 & 3-15 & 30 & 0.845 & \mathrm{n} / \mathrm{a} & \log & \mathrm{n} / \mathrm{a} & \mathrm{comp} & \text { Maine } \\ 0.0042 & 2.9740 & 1-50^{\circ} & 14 & 0.974 & 0.232 & \log & 1.064 & \mathrm{n} / \mathrm{a} & \text { New Hampshire } \\ 0.0104 & 2.5515 & 3-66^{\circ} & 42 & 0.937 & \mathrm{n} / \mathrm{a} & \ln & \mathrm{n} / \mathrm{a} & \text { comp } & \text { Maine }\end{array}$

Oak, black (Quercus velutina Lam.)

\begin{tabular}{|c|c|c|c|c|c|c|c|c|c|c|}
\hline \multirow[t]{2}{*}{$\mathrm{AB}:$} & 0.0904 & 2.5143 & $7-27$ & 27 & 0.983 & 0.121 & In & 1.007 & comp & Rhode Island \\
\hline & 0.0945 & 2.5030 & $5-40^{a}$ & 19 & 0.994 & 2268 & abs & $\mathrm{n} / \mathrm{a}$ & $223(50)$ & West Virginia \\
\hline SW: & 0.2263 & 2.1285 & $29-88$ & 26 & 0.950 & $\mathrm{n} / \mathrm{a}$ & $\log$ & $\mathrm{n} / \mathrm{a}$ & comp & $\begin{array}{l}\text { Kentucky, Nort } \\
\text { Carolina, Tenn }\end{array}$ \\
\hline SB: & 0.0769 & 1.8481 & $29-88$ & 26 & 0.840 & $n / a$ & $\log$ & $\mathrm{n} / \mathrm{a}$ & comp & $\begin{array}{l}\text { Kentucky, Nort } \\
\text { Carolina, Tenn }\end{array}$ \\
\hline ST: & 0.2767 & 2.1081 & $29-88$ & 26 & 0.950 & $n / a$ & $\log$ & $\mathrm{n} / \mathrm{a}$ & comp & $\begin{array}{l}\text { Kentucky, Nort } \\
\text { C'arolina. Tenn }\end{array}$ \\
\hline \multicolumn{11}{|c|}{ Oak, chestnut (Quercus prinus L.) } \\
\hline \multirow[t]{2}{*}{$\mathrm{AB}:$} & 0.0554 & 2.7276 & $5-50$ & 13 & 0.990 & $\mathrm{n} / \mathrm{a}$ & $\log$ & $n / a$ & $n / a$ & West Virginia \\
\hline & 0.0907 & 2.5344 & $5-40^{a}$ & 19 & 0.991 & 29.48 & abs & $n / a$ & $22.3(50$ & West Virginia \\
\hline ST: & 0.0741 & 2.5226 & $5-40^{a}$ & 19 & 0.983 & 31.33 & abs & $n / a$ & $22.3(50)$ & West Virginia \\
\hline
\end{tabular}

Oak, mossy-cup (Quercus macrocarpa Michx.)

$\begin{array}{lllllllllll}\text { AB: } & 0.1447 & 2.2820 & 6-25 & 9 & 0.978 & 0.205 & \ln & 1.021 & \mathrm{n} / \mathrm{a} & \text { Upper Great Lakes } \\ \mathrm{SW}: & 0.0636 & 2.3980 & 6-25 & 9 & 0.977 & 0.223 & \ln & 1.025 & \mathrm{n} / \mathrm{a} & \text { Upper Great Lakes } \\ \mathrm{SB}: & 0.0303 & 2.0640 & 6-25 & 9 & 0.993 & 0.102 & \ln & 1.005 & \mathrm{n} / \mathrm{a} & \text { Upper Great Lakes } \\ \mathrm{FL}: & 0.3129 & 0.6681 & 6-25 & 9 & 0.392 & 0.559 & \mathrm{ln} & 1.169 & \mathrm{n} / \mathrm{a} & \text { Upper Great Lakes }\end{array}$

Oak, red (Quercus rubra L.)

$\begin{array}{llllrllllll}\text { AB: } & 0.1130 & 2.4572 & 5-50 & 24 & 0.950 & \mathrm{n} / \mathrm{a} & \log & \mathrm{n} / \mathrm{a} & \mathrm{n} / \mathrm{a} & \text { West Virginia } \\ & 0.1335 & 2.4220 & 5-34 & 16 & 0.993 & 0.134 & \mathrm{ln} & 1.009 & \mathrm{n} / \mathrm{a} & \text { Upper Great Lakes } \\ & 0.0643 & 2.6598 & 5-40 & 19 & 0.988 & 35.87 & \text { ahs } & \mathrm{n} / \mathrm{a} & 22.3(50) \text { West Virginia } \\ \mathrm{SW}: & 0.0950 & 2.3628 & 5-34 & 16 & \mathrm{n} / \mathrm{a} & \mathrm{n} / \mathrm{a} & \mathrm{calc} & \mathrm{n} / \mathrm{a} & \mathrm{n} / \mathrm{a} & \text { Upper Great Lakes } \\ \mathrm{SB}: & 0.0130 & 2.4546 & 5-34 & 16 & \mathrm{n} / \mathrm{a} & \mathrm{n} / \mathrm{a} & \mathrm{calc} & \mathrm{n} / \mathrm{a} & \mathrm{n} / \mathrm{a} & \text { Upper Great Lakes } \\ \mathrm{ST}: & 0.1356 & 2.3600 & 1-44 & 39 & 0.992 & 0.217 & \mathrm{ln} & 1.024 & \mathrm{comp} & \text { Ncw Hampshirc } \\ & 0.0973 & 2.4770 & 4-11 & 8 & 0.937 & 0.255 & \mathrm{ln} & 1.033 & \mathrm{n} / \mathrm{a} & \text { New Hampshire } \\ & & & & & & & & & & \\ \text { FL: } & 0.0238 & 1.8600 & 1-44 & 14 & 0.957 & 0.556 & \mathrm{ln} & 1.167 & \mathrm{comp} & \text { New Hampshire } \\ & 0.0102 & 2.1870 & 4-11 & 8 & 0.743 & 0.512 & \mathrm{ln} & 1.140 & \mathrm{n} / \mathrm{a} & \text { New Hampshire } \\ & 0.0480 & 1.4550 & 5-34 & 16 & 0.835 & 0.426 & \mathrm{ln} & 1.095 & \mathrm{n} / \mathrm{a} & \text { Upper Great Lakes } \\ \mathrm{BR}: & 0.0122 & 2.6300 & 1-44 & 39 & 0.916 & 0.822 & \mathrm{ln} & 1.402 & \mathrm{comp} & \text { New Hampshire } \\ & 0.0060 & 2.9090 & 4-11 & 8 & 0.956 & 0.243 & \mathrm{ln} & 1.030 & \mathrm{n} / \mathrm{a} & \text { New Hampshire }\end{array}$

Oak, scarlet (Quercus coccinea Muench.)

\begin{tabular}{|c|c|c|c|c|c|c|c|c|c|c|}
\hline $\mathrm{AB}$ : & $\begin{array}{l}0.0536 \\
0.2482\end{array}$ & $\begin{array}{l}2.7147 \\
2.1900\end{array}$ & $\begin{array}{l}8-28 \\
0-23\end{array}$ & $\begin{array}{l}15 \\
15\end{array}$ & $\begin{array}{l}0.978 \\
0.988\end{array}$ & $\begin{array}{l}0.125 \\
0.050\end{array}$ & $\begin{array}{l}\text { In } \\
\log \end{array}$ & $\begin{array}{l}1.008 \\
1.003\end{array}$ & $\begin{array}{l}\text { comp } \\
\mathrm{n} / \mathrm{a}\end{array}$ & $\begin{array}{l}\text { Rhode Island } \\
\text { New York }\end{array}$ \\
\hline & 0.1241 & 2.4395 & $5-40^{a}$ & 19 & 0.993 & 27.22 & abs & $\mathrm{n} / \mathrm{a}$ & $22.3(50)$ & West Virginia \\
\hline SW: & 0.1000 & 2.3025 & $0-23$ & 15 & 0.990 & 0.081 & $\log$ & 1.008 & $\mathrm{n} / \mathrm{a}$ & New York \\
\hline & 0.0405 & 2.6479 & $5-40^{a}$ & 19 & 0.978 & 30.87 & abs & $\mathrm{n} / \mathrm{a}$ & $22.3(50)$ & West Virginia \\
\hline SB: & 0.0475 & 1.9909 & $0-23$ & 15 & 0.994 & 0.062 & $\log$ & 1.004 & $n / a$ & New York \\
\hline ST: & 0.1434 & 2.2391 & $0-23$ & 15 & 0.994 & 0.074 & $\log$ & 1.006 & $\mathrm{n} / \mathrm{a}$ & New York \\
\hline & 0.0517 & 2.6160 & $5-40^{a}$ & 19 & 0.979 & 34.50 & abs & $n / a$ & $22.3(50)$ & West Virginia \\
\hline
\end{tabular}

Perala and Alban, 1994

Young et al., 1980

Freedman et al. 1982

Hocker and Earley, 1983

Ker. 1980a

Pastor and Bockheim, 1981

Ribe, 1973

Whittaker et al. 1974

Young et al., 1980

Bridge. 1979

Wiant et al.. 1977

King and Schnell. 1972

King and Schnell. 1972

King and Schnell, 1972

Brenneman et at., 1978

Wiant et al., $1977^{\text {* }}$

Wiant et al, 1977

Perala and Alban. 1994

Perala and Alban. 1994

Perala and Alban, 1994

Perala and Alban; 1994

Brenneman et al., 1978

Perala and Alban, 1994

Wiant et al.. 1977 d

Perala and Alban. 1994

Perala and Alban, 1994

Hocker and Earley, 1983

Kinerson and

Bartholomew, 1977

Hocker and Earley, 1983

Kinerson and Bartholomew, 1977

Perala and Alban, 1994

Hocker and Earley, 1983

Kinerson and Bartholomew, 1977

Bridge, $1979^{h}$

Whittaker and

Woodwell, 1968

Wiant et al., 1977

Whittaker and

Woodwell, 1968

Wiant et al.. 1977

Whittaker and

Woodwell, 1968

Whittaker and

Woodwell. 1968

Wiant et al., 1977 


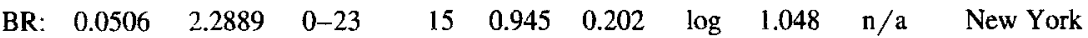

Oak, white (Quercus alba L.)

\begin{tabular}{|c|c|c|c|c|c|c|c|c|c|c|}
\hline \multirow[t]{3}{*}{$\mathrm{AB}:$} & 0.0579 & 2.6887 & $5-50$ & 29 & 0.950 & $n / a$ & $\log$ & $\mathrm{n} / \mathrm{a}$ & $\mathrm{n} / \mathrm{a}$ & West Virginia \\
\hline & 0.0293 & 2.8661 & $8-26$ & 22 & 0.937 & 0.285 & $\ln$ & 1.042 & comp & Rhode Island \\
\hline & 0.2022 & 2.1666 & $0-18$ & 15 & 0.986 & 0.078 & $\log$ & 1.007 & $\mathrm{n} / \mathrm{a}$ & New York \\
\hline \multirow{3}{*}{ SW: } & 0.0472 & 2.7010 & $5-40^{a}$ & 19 & 0.988 & 32.66 & abs & $\mathrm{n} / \mathrm{a}$ & $22.3(50)$ & West Virginia \\
\hline & 0.0914 & 2.2537 & $0-18$ & 15 & 0.990 & 0.070 & $\log$ & 1.006 & $\mathrm{n} / \mathrm{a}$ & New York \\
\hline & 0.0486 & 2.5956 & $5-40^{a}$ & 19 & 0.989 & 22.25 & abs & $\mathrm{n} / \mathrm{a}$ & $22.3(50)$ & West Virginia \\
\hline SB & 0.0510 & 1.9747 & $0-18$ & 15 & 0.996 & 0.042 & $\log$ & 1.002 & $\mathrm{n} / \mathrm{a}$ & New York \\
\hline \multirow[t]{2}{*}{ ST: } & 0.1392 & 2.1844 & $0-18$ & 15 & 0.994 & 0.061 & $\log$ & 1.004 & $n / a$ & New York \\
\hline & 0.0487 & 2.6279 & $5-40^{a}$ & 19 & 0.989 & 24.97 & abs & $\mathrm{n} / \mathrm{a}$ & $22.3(50)$ & West Virginia \\
\hline BR. & 0.0274 & 2.3371 & $0-18$ & 15 & 0.916 & 0.211 & $\log$ & 1.053 & $\mathrm{n} / \mathrm{a}$ & New York \\
\hline
\end{tabular}

Pine, eastern white (Pinus strobus L.)

\begin{tabular}{|c|c|c|c|c|c|c|c|c|c|c|}
\hline \multirow[t]{4}{*}{$\mathrm{AB}$ : } & 0.1617 & 2.1420 & $2-37$ & 47 & 0.968 & 0.243 & In & 1.030 & $\mathrm{n} / \mathrm{a}$ & New Brunswick \\
\hline & 0.6298 & 1.3475 & $0-15$ & 10 & 0.850 & $\mathrm{n} / \mathrm{a}$ & $\log$ & $\mathrm{n} / \mathrm{a}$ & $\mathrm{n} / \mathrm{a}$ & New Brunswick \\
\hline & 0.0755 & 2.3833 & $5-26$ & 12 & $\mathrm{n} / \mathrm{a}$ & $\mathrm{n} / \mathrm{a}$ & calc & $\mathrm{n} / \mathrm{a}$ & $\mathrm{n} / \mathrm{a}$ & Upper Great Lakes \\
\hline & 0.0696 & 2.4490 & $3-66^{a}$ & 35 & $\mathrm{n} / \mathrm{a}$ & $\mathrm{n} / \mathrm{a}$ & calc & $n / a$ & comp & Maine \\
\hline \multirow[t]{2}{*}{ SW: } & 0.0298 & 2.5979 & $2-37$ & 47 & 0.976 & 0.243 & $\ln$ & 1.030 & $\mathrm{n} / \mathrm{a}$ & New Brunswick \\
\hline & 0.0394 & 2.2935 & $5-26$ & 12 & $\mathrm{n} / \mathrm{a}$ & $\mathrm{n} / \mathrm{a}$ & calc & $\mathrm{n} / \mathrm{a}$ & $\mathrm{n} / \mathrm{a}$ & Upper Great Lakes \\
\hline \multirow[t]{2}{*}{ SB: } & 0.0152 & 2.1781 & $2-37$ & 47 & 0.949 & 0.312 & $\ln$ & 1.050 & $\mathrm{n} / \mathrm{a}$ & New Brunswick \\
\hline & 0.0079 & 2.2080 & $5-26$ & 12 & 0.986 & 0.155 & ln & 1.012 & $n / a$ & Upper Great Lakes \\
\hline \multirow[t]{6}{*}{ ST: } & 0.0546 & 2.4200 & $2-26$ & 33 & 0.979 & 0.235 & $\ln$ & 1.028 & comp & New Hampshire \\
\hline & 0.0414 & 2.5360 & $2-37$ & 47 & 0.973 & 0.243 & $\ln$ & 1.030 & $\mathrm{n} / \mathrm{a}$ & New Brunswick \\
\hline & 0.0718 & 2.2690 & $3-20$ & 23 & 0.974 & 0.195 & $\ln$ & 1.019 & $\mathrm{n} / \mathrm{a}$ & New Hampshire \\
\hline & 0.1735 & 1.7076 & $0-15$ & 10 & 0.949 & $\mathrm{n} / \mathrm{a}$ & $\log$ & $\mathrm{n} / \mathrm{a}$ & $\mathrm{n} / \mathrm{a}$ & New Brunswick \\
\hline & 0.0615 & 2.1338 & $1-18$ & 20 & 0.988 & 0.144 & $\ln$ & 1.024 & $\mathrm{n} / \mathrm{a}$ & North Carolina \\
\hline & 0.0404 & 2.5459 & $3-66^{a}$ & 35 & 0.988 & $\mathrm{n} / \mathrm{a}$ & $\ln$ & $n / a$ & comp & Maine \\
\hline \multirow[t]{6}{*}{ FL: } & 0.0250 & 1.7500 & $2-26$ & 10 & 0.954 & 0.371 & $\ln$ & 1.071 & comp & New Hampshire \\
\hline & 0.0677 & 1.4653 & $2-37$ & 47 & 0.781 & 0.312 & $\ln$ & 1.050 & $\mathrm{n} / \mathrm{a}$ & New Brunswick \\
\hline & 0.0004 & 2.5860 & $3-20$ & 23 & 0.767 & 0.745 & $\ln$ & 1.320 & $\mathrm{n} / \mathrm{a}$ & New Hampshire \\
\hline & 0.0039 & 1.8170 & $5-26$ & 12 & 0.898 & 0.262 & In & 1.035 & $\mathrm{n} / \mathrm{a}$ & Upper Great Lakes \\
\hline & 0.0211 & 2.1354 & $1-18$ & 20 & 0.970 & 0.225 & $\ln$ & 1.026 & $\mathrm{n} / \mathrm{a}$ & North Carolina \\
\hline & 0.0183 & 1.9674 & $3-66^{a}$ & 35 & 0.963 & $n / a$ & $\ln$ & $n / a$ & comp & Maine \\
\hline \multirow[t]{5}{*}{ BR: } & 0.0318 & 1.9700 & $2-26$ & 10 & 0.976 & 0.301 & $\ln$ & 1.046 & comp & New Hampshire \\
\hline & 0.0709 & 1.7086 & $2-37$ & 47 & 0.783 & 0.392 & $\ln$ & 1.080 & $\mathrm{n} / \mathrm{a}$ & New Brunswick \\
\hline & 0.0057 & 2.6560 & $3-20$ & 23 & 0.859 & 0.565 & $\ln$ & 1.173 & $\mathrm{n} / \mathrm{a}$ & New Hampshire \\
\hline & 0.0235 & 2.5328 & $1-18$ & 20 & 0.980 & 0.173 & $\ln$ & 1.015 & $\mathbf{n} / \mathbf{a}$ & North Carolina \\
\hline & 0.0030 & 2.4858 & $3-66^{a}$ & 35 & 0.953 & $n / a$ & $\ln$ & $\mathrm{n} / \mathrm{a}$ & comp & Maine \\
\hline
\end{tabular}

Pine, jack (Pinus banksiana Lamb.)

$\begin{array}{lllllllllll}\mathrm{AB}: & 0.1520 & 2.2730 & \mathrm{n} / \mathrm{a} & 20 & 0.978 & 3.820 & \mathrm{abs} & \mathrm{n} / \mathrm{a} & 12.2(50) \text { Minnesota } \\ 0.0919 & 2.4206 & 2-32 & 77 & 0.986 & \mathrm{n} / \mathrm{a} & \log & \mathrm{n} / \mathrm{a} & \text { comp } & \text { Ontario } \\ 0.1093 & 2.3291 & 3-34 & 42 & 0.980 & 0.141 & \ln & 1.010 & \mathrm{n} / \mathrm{a} & \text { Nova Scotia } \\ 0.2131 & 2.1283 & 0-38 & 195 & 0.978 & 23.20 & \mathrm{abs} & \mathrm{n} / \mathrm{a} & \mathrm{comp} & \text { New Brunswick, } \\ & & & & & & & & & \text { Nova Scotia } \\ & 0.2186 & 1.9400 & 0-20 & 42 & 0.914 & \mathrm{n} / \mathrm{a} & \log & \mathrm{n} / \mathrm{a} & \mathrm{n} / \mathrm{a} & \text { New Brunswick } \\ 0.1747 & 2.2495 & 6-39 & 41 & \mathrm{n} / \mathrm{a} & \mathrm{n} / \mathrm{a} & \text { calc } & \mathrm{n} / \mathrm{a} & \mathrm{n} / \mathrm{a} & \text { Upper Great Lakes }\end{array}$

Whittaker and

Woodwell, 1968

Brenneman et al., 1978

Bridge, $1979^{\mathrm{b}}$

Whittaker and

Woodwell, 1968

Wiant et al., $1977^{\circ}$

Whittaker and

Woodwell, 1968

Wiant et al., 1977

Whittaker and

Woodwell, 1968

Whittaker and

Woodwell, 1968

Wiant et al., 1977

Whittaker and

Woodwell, 1968

Ker, $1980 \mathrm{a}$

MacLean and Wein, 1976

Perala and Alban, 1994

Young et al., 1980

Ker, 1980a

Perala and Alban, 1994

Ker, 1980a

Perala and Alban, 1994

Hocker and Earley, 1983

Ker, 1980a

Kinerson and

Bartholomew, 1977

MacLean and Wein, 1976

Swank and Schreuder, 1974

Young et al., 1980

Hocker and Earley, 1983

Ker, 1980a

Kinerson and

Bartholonew, 1977

Perala and Alban, 1994

Swank and Schreuder, 1974

Young et al., 1980

Hocker and Earley, 1983

Ker, 1980a

Kinerson and

Bartholomew, 1977

Swank and Schreuder, 1974

Young et al., 1980

Crow, 1971

Hegyi, 1972

Ker, 1980b

Ker, 1984

MacLean and Wein, 1976

Perala and Alban, 1994 


\begin{tabular}{|c|c|c|c|c|c|c|c|c|c|c|c|}
\hline \multicolumn{2}{|c|}{ SSW: 0.0826} & 2.2860 & $\mathrm{n} / \mathrm{a}$ & 40 & 0.950 & 3.141 & $a b s$ & $n / a$ & $12.2(50)$ & Minnesota & Crow. 1971 \\
\hline & 0.0402 & 2.5578 & $3-34$ & 42 & 0.970 & 0.141 & $\ln$ & 1.010 & $\mathrm{n} / \mathrm{a}$ & Nova Scotia & Ker, $1980 \mathrm{~h}$ \\
\hline & 0.1172 & 2.2116 & $0-38$ & 195 & 0.971 & 19.30 & $\mathrm{abs}$ & $n / a$ & comp & $\begin{array}{l}\text { New Brunswick, } \\
\text { Nova Scotia }\end{array}$ & Ker, 1984 \\
\hline & 0.1543 & 2.1839 & $6-39$ & 41 & $n / a$ & $n / a$ & calc & $\mathrm{n} / \mathrm{a}$ & $\mathrm{n} / \mathrm{a}$ & Upper Great Lakes & Perala and Alban. 1994 \\
\hline \multirow[t]{2}{*}{ SW: } & 0.0190 & 1.9916 & $3-34$ & 42 & 0.980 & 0.141 & $\ln$ & 1.010 & $n / a$ & Nova Scotia & Ker, $1980 \mathrm{~b}$ \\
\hline & 0.0321 & 1.9164 & $6-39$ & 41 & $\mathrm{n} / \mathrm{a}$ & $\mathrm{n} / \mathrm{a}$ & calc & $\mathrm{n} / \mathrm{a}$ & $\mathrm{n} / \mathrm{a}$ & Upper Great Lakes & Perala and Alban. 1994 \\
\hline \multirow[t]{4}{*}{ ST: } & 0.0283 & 2.7144 & $2-32$ & 77 & 0.947 & $n / a$ & $\log$ & $\mathrm{n} / \mathrm{a}$ & comp & Ontario & Hegyı, 1972 \\
\hline & 0.0538 & 2.4883 & $3-34$ & 42 & 0.980 & 0.141 & $\ln$ & 1.010 & $\mathrm{n} / \mathrm{a}$ & Nova Scotia & Ker, $1980 \mathrm{~b}$ \\
\hline & 0.1470 & 2.1673 & $0-38$ & 195 & 0.972 & 20.50 & abs & $n / a$ & comp & $\begin{array}{l}\text { New Brunswick, } \\
\text { Nova Scotia }\end{array}$ & Ker, 1984 \\
\hline & 0.1064 & 2.1366 & $0-20$ & 42 & 0.950 & $\mathrm{n} / \mathrm{a}$ & $\log$ & $\mathrm{n} / \mathrm{a}$ & $n / a$ & New Brunswick & MacLean and Wein, 1976 \\
\hline \multirow[t]{4}{*}{ FL: } & 0.0372 & 1.7188 & $2-32$ & 77 & 0.947 & $\mathrm{n} / \mathrm{a}$ & $\log$ & $n / a$ & comp & Ontario & Hegyi, 1972 \\
\hline & 0.0138 & 2.0512 & $3-34$ & 42 & 0.870 & 0.392 & $\ln$ & 1.080 & $\mathrm{n} / \mathrm{a}$ & Nova Scotia & Ker. $1980 \mathrm{~b}$ \\
\hline & 0.0489 & 1.7140 & $0-38$ & 195 & 0.870 & 3.500 & abs & $\mathrm{n} / \mathrm{a}$ & comp & $\begin{array}{l}\text { New Brunswick, } \\
\text { Nova Scotia }\end{array}$ & Ker. 1984 \\
\hline & 0.0009 & 2.903 & $6-39$ & 40 & 0.829 & 0.510 & $\ln$ & 1.139 & $\mathrm{n} / \mathrm{a}$ & Upper Great Lakes & Perala and Alban, 1994 \\
\hline \multirow[t]{3}{*}{ BR: } & 0.0022 & 2.9810 & $\mathrm{n} / \mathrm{a}$ & 40 & 0.955 & 0.671 & $\mathrm{abs}$ & $\mathrm{n} / \mathrm{a}$ & $12.2(50)$ & Minnesota & Crow, 1971 \\
\hline & 0.0181 & 2.2443 & $3-34$ & 42 & 0.820 & 0.341 & $\ln$ & 1.060 & $\mathrm{n} / \mathrm{a}$ & Nova Scotia & Ker, $1980 \mathrm{~b}$ \\
\hline & 0.0353 & 2.1113 & $0-38$ & 195 & 0.845 & 10.30 & $\mathrm{abs}$ & $\mathrm{n} / \mathrm{a}$ & comp & $\begin{array}{l}\text { New Brunswick, } \\
\text { Nova Scotia }\end{array}$ & Ker, 1984 \\
\hline
\end{tabular}

Pine, loblolly (Pinus taeda L.)

$\begin{array}{llllrllllll}\text { ST: } & 0.0420 & 2.5090 & 10-30 & 26 & 0.95 & 0.138 & \ln & 1.010 & \mathrm{n} / \mathrm{a} & \text { North Carolina } \\ \text { FL: } & 0.0103 & 2.1741 & 13-43 & 112 & \mathrm{n} / \mathrm{a} & \mathrm{n} / \mathrm{a} & \mathrm{ln} & \mathrm{n} / \mathrm{a} & \text { comp } & \text { Louisiana } \\ & 0.0012 & 2.8432 & 10-30 & 26 & 0.920 & 0.202 & \mathrm{ln} & 1.021 & \mathrm{n} / \mathrm{a} & \text { North Carolina } \\ \mathrm{BR}: & 0.0022 & 2.8687 & 13-43 & 112 & \mathrm{n} / \mathrm{a} & \mathrm{n} / \mathrm{a} & \mathrm{ln} & \mathrm{n} / \mathrm{a} & \text { comp } & \text { Louisiana } \\ & 0.0003 & 3.4981 & 10-30 & 26 & 0.894 & 0.290 & \mathrm{ln} & 1.043 & \mathrm{n} / \mathrm{a} & \text { North Carolina }\end{array}$

Raiston, 1973

Baldwin, $1989^{\mathrm{i}}$

Ralston, 1973

Baldwin, $1989^{i}$

Ralston, 1973

Pine, lodgepole ( Pinus contorta Dougl.)

$\begin{array}{llllrllllll}\text { ST: } & 0.3377 & 0.9774 & 1-5 & 8 & 0.858 & 0.255 & \text { In } & 1.033 & \text { comp } & \text { ldaho, Montana } \\ & 0.0492 & 2.4287 & 3-29 & 19 & 0.980 & 0.228 & \text { In } & 1.026 & \mathrm{n} / \mathrm{a} & \text { Colorado } \\ \text { FL: } & 0.2508 & 0.6976 & 1-5 & 8 & 0.659 & 0.322 & \text { In } & 1.053 & \text { comp } & \text { Idaho, Montana } \\ & 0.0239 & 1.8362 & 3-29 & 19 & 0.840 & 0.480 & \text { In } & 1.122 & \mathrm{n} / \mathrm{a} & \text { Colorado } \\ \text { BR: } & 0.2764 & 0.7313 & 1-5 & 8 & 0.626 & 0.362 & \text { In } & 1.068 & \mathrm{comp} & \text { Idaho, Montana } \\ & 0.0089 & 2.3533 & 3-29 & 19 & 0.890 & 0.493 & \mathrm{ln} & 1.129 & \mathrm{n} / \mathrm{a} & \text { Colorado }\end{array}$

Brown, 1978

Gholz et al., 1979

Brown, 1978 "

Gholz et al., 1979

Brown. 1978 b

Gholz et al., 1979

Pine, pitch (Pinus rigidu Mill.)

$\begin{array}{llllllllllll}\text { AB: } & 0.1040 & 2.3373 & 0-31 & 15 & 0.996 & 0.045 & \log & 1.002 & \mathrm{n} / \mathrm{a} & \text { New York } \\ \text { SW: } & 0.0528 & 2.3779 & 0-31 & 15 & 0.994 & 0.066 & \log & 1.005 & \mathrm{n} / \mathrm{a} & \text { New York } \\ \text { SB: } & 0.0242 & 2.1249 & 0-31 & 15 & 0.974 & 0.101 & \log & 1.012 & \mathrm{n} / \mathrm{a} & \text { New York } \\ \text { ST: } & 0.0751 & 2.3261 & 0-31 & 15 & 0.994 & 0.062 & \log & 1.004 & \mathrm{n} / \mathrm{a} & \text { New York } \\ \text { BR: } & 0.0129 & 2.5516 & 0-31 & 15 & 0.976 & 0.120 & \log & 1.017 & \mathrm{n} / \mathrm{a} & \text { New York }\end{array}$

Whittaker and Woodwell, 1968 Whittaker and Woodwell, 1968 Whittaker and Woodwell. 1968 Whittaker and Woodwell. 1968 Whittaker and Woodwell, 1968

Pine, ponderosa ( inus ponderosa Laws.)

$\begin{array}{llllrllllll}\text { SW: } & 0.0110 & 2.7587 & 16-80 & 9 & 0.990 & 0.176 & \ln & 1.016 & \mathrm{n} / \mathrm{a} & \text { Arizona } \\ \text { SB: } & 0.0144 & 2.2312 & 16-80 & 9 & 0.970 & 0.251 & \ln & 1.032 & \mathrm{n} / \mathrm{a} & \text { Arizona } \\ \text { ST: } & 0.2679 & 1.4726 & 1-11 & 25 & 0.886 & 0.463 & \ln & 1.113 & \text { comp } & \text { Idaho, Montana } \\ \text { FL: } & 0.1167 & 1.5774 & 2-86 & 31 & 0.954 & 0.461 & \ln & 1.112 & \text { comp } & \text { Idaho, Montana } \\ & 0.0286 & 1.9920 & 5-39 & 23 & 0.972 & 0.209 & \ln & 1.022 & 33.5(50) \text { Oregon } \\ & 0.0119 & 2.0967 & 16-80 & 9 & 0.840 & 0.581 & \ln & 1.184 & \mathrm{n} / \mathrm{a} & \text { Arizona }\end{array}$

Gholz et al. 1979 Gholz et al., 1979 Brown, $1978^{b}$ Brown, $1978^{b}$ Cochran et al.. 1984 Gholz et al., 1979 
BR: 0 $\begin{array}{lllllll}0.0469 & 2.1315 & 2-86 & 31 & 0.962 & 0.563 & 1\end{array}$ $\begin{array}{lllllll}0.0096 & 2.4645 & 5-39 & 23 & 0.941 & 0.381 & \mathrm{l}\end{array}$ $0.0045 \quad 2.7185 \quad 16-80$
1.172

1.075

1.021 comp Idaho, Montana

33.5(50) Oregon

$\mathrm{n} / \mathrm{a} \quad$ Arizona
Brown, $1978^{b}$

Cochran et al., 1984

Gholz et al., 1979

Pine, red (Pinus resinosa Ait.)

$\begin{array}{lllllllllll}\text { AB: } & 0.0847 & 2.3503 & 2-34 & 47 & 0.990 & 0.141 & \ln & 1.010 & \mathrm{n} / \mathrm{a} & \text { Nova Scotia } \\ & 0.0778 & 2.4171 & 3-46 & 69 & \mathrm{n} / \mathrm{a} & \mathrm{n} / \mathrm{a} & \mathrm{calc} & \mathrm{n} / \mathrm{a} & \mathrm{n} / \mathrm{a} & \text { Upper Great Lakes } \\ & 0.1003 & 2.3865 & 3-51^{\mathrm{a}} & 14 & \mathrm{n} / \mathrm{a} & \mathrm{n} / \mathrm{a} & \mathrm{calc} & \mathrm{n} / \mathrm{a} & \mathrm{comp} & \text { Maine } \\ \mathrm{SW}: & 0.0448 & 2.4418 & 2-34 & 47 & 0.990 & \mathrm{n} / \mathrm{a} & \ln & 1.00 & \mathrm{n} / \mathrm{a} & \text { Nova Scotia } \\ & 0.0649 & 2.3496 & 3-46 & 69 & \mathrm{n} / \mathrm{a} & \mathrm{n} / \mathrm{a} & \mathrm{calc} & \mathrm{n} / \mathrm{a} & \mathrm{n} / \mathrm{a} & \text { Upper Great Lakes } \\ \mathrm{SB}: & 0.0157 & 2.0701 & 2-34 & 47 & 0.990 & 0.141 & \ln & 1.010 & \mathrm{n} / \mathrm{a} & \text { Nova Scotia } \\ & 0.0141 & 2.0900 & 3-46 & 69 & 0.990 & 0.111 & \ln & 1.006 & \mathrm{n} / \mathrm{a} & \text { Upper Great Lakes } \\ \mathrm{ST}: & 0.0586 & 2.3892 & 2-34 & 47 & 0.990 & \mathrm{n} / \mathrm{a} & \ln & 1.00 & \mathrm{n} / \mathrm{a} & \text { Nova Scotia } \\ & 0.0631 & 2.4481 & 3-51^{\mathrm{a}} & 14 & 0.983 & \mathrm{n} / \mathrm{a} & \ln & \mathrm{n} / \mathrm{a} & \mathrm{comp} & \text { Maine } \\ \mathrm{FL}: & 0.0120 & 2.1220 & 2-34 & 47 & 0.930 & 0.280 & \ln & 1.040 & \mathrm{n} / \mathrm{a} & \text { Nova Scotia } \\ & 0.0007 & 3.1220 & 3-46 & 69 & 0.895 & 0.553 & \ln & 1.165 & \mathrm{n} / \mathrm{a} & \text { Upper Great Lakes } \\ & 0.0177 & 2.1803 & 3-51^{\mathrm{a}} & 14 & 0.899 & \mathrm{n} / \mathrm{a} & \ln & \mathrm{n} / \mathrm{a} & \mathrm{comp} & \text { Maine } \\ \mathrm{BR}: & 0.0079 & 2.4631 & 2-34 & 47 & 0.910 & 0.368 & \ln & 1.070 & \mathrm{n} / \mathrm{a} & \text { Nova Scotia } \\ & 0.0098 & 2.5011 & 3-51^{\mathrm{a}} & 14 & 0.986 & \mathrm{n} / \mathrm{a} & \ln & \mathrm{n} / \mathrm{a} & \mathrm{comp} & \text { Maine }\end{array}$

Ker, 1980b

Perala and Alban, 1994

Young et al., 1980

Ker, 1980b

Perala and Alban, 1994

Ker, 1980b

Perala and Alban, 1994

Ker, $1980 b$

Young et al., 1980

Ker, 1980b

Perala and Alban, 1994

Young et al., 1980

Ker, 1980b

Young et al., 1980

Pine, sugar ( Pinus lambertiana Dougl.)

$\begin{array}{lllllllllll}\text { SW: } & 0.0183 & 2.6667 & 21-43 & 5 & 0.960 & 0.195 & \ln & 1.019 & \text { comp } & \text { Oregon } \\ \text { SB: } & 0.0048 & 2.6186 & 21-43 & 5 & 0.910 & 0.285 & \ln & 1.041 & \text { comp } & \text { Oregon } \\ \text { FL: } & 0.0144 & 2.0327 & 21-43 & 5 & 0.520 & 0.662 & \ln & 1.245 & \text { comp } & \text { Oregon } \\ \text { BR: } & 0.0004 & 3.3648 & 21-43 & 5 & 0.810 & 0.548 & \ln & 1.162 & \text { comp } & \text { Oregon }\end{array}$

Gholz et al., 1979

Gholz et al., 1979

Cholz et al., 1979

Gholz et al., 1979

Pine, western white (Pinus monticola Dougl.)

$\begin{array}{lllllllllll}\text { ST: } & 0.2953 & 1.4073 & 1-12 & 14 & 0.905 & 0.373 & \text { In } & 1.072 & \text { comp } & \text { Idaho, Montana } \\ \text { FL: } & 0.2761 & 1.0684 & 1-19 & 16 & 0.898 & 0.343 & \text { In } & 1.061 & \text { comp } & \text { Idaho, Montana } \\ \text { BR: } & 0.2312 & 1.1825 & 1-19 & 16 & 0.891 & 0.393 & \text { In } & 1.080 & \text { comp } & \text { Idaho, Montana }\end{array}$

Brown, $1978^{b}$

Brown, $1978^{b}$

Brown, $1978^{b}$

Pine, whitebark (Pinus albicaulis Engelm.)

$\begin{array}{lllllllllll}\text { ST: } & 0.3110 & 1.4603 & 1-12 & 10 & 0.876 & 0.582 & \text { In } & 1.184 & \text { comp } & \text { Idaho, Montana } \\ \text { FL: } & 0.1168 & 1.2751 & 1-19 & 13 & 0.884 & 0.525 & \text { In } & 1.147 & \text { comp } & \text { Idaho, Montana } \\ \text { RR: } & 0.1164 & 1.3767 & 1-19 & 13 & 0.887 & 0.559 & \text { In } & 1.169 & \text { comp } & \text { Idaho, Montana }\end{array}$

Brown, $1978^{b}$ Brown, $1978^{b}$

Brown, $1978^{b}$

Poplar, yellow ( Liriodendron tulipifera L.)

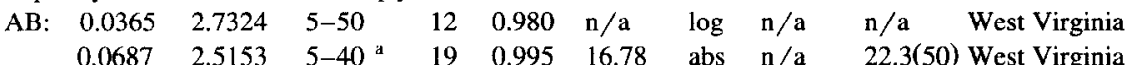

Brenneman et al., 1978

Wiant et al., $1977^{\mathrm{d}}$

Spruce, black ( Picea mariana (Mill.) B.S.P.)

\begin{tabular}{|c|c|c|c|c|c|c|c|c|c|c|}
\hline \multirow[t]{8}{*}{$\mathrm{AB}:$} & 0.2626 & 2.0707 & $2-30$ & 24 & 0.983 & 0.227 & $\ln$ & 1.026 & $\mathrm{n} / \mathrm{a}$ & Nova Scotia \\
\hline & 0.1530 & 2.2480 & $1-23$ & 24 & 0.990 & 4.630 & abs & $\mathrm{n} / \mathrm{a}$ & $8.2(50)$ & Minnesota \\
\hline & 0.1683 & 2.1777 & $2-34$ & 49 & 0.990 & 0.199 & $\ln$ & 1.020 & $\mathrm{n} / \mathrm{a}$ & Nova Scotia \\
\hline & 0.1444 & 2.2604 & $0-37$ & 195 & 0.978 & 26.20 & abs & $\mathrm{n} / \mathrm{a}$ & comp & $\begin{array}{l}\text { New Brunswick, } \\
\text { Nova Scotia }\end{array}$ \\
\hline & 1.3836 & 1.5440 & $2-15$ & 12 & 0.831 & 0.178 & $\log$ & 1.037 & comp & Subarctic Québec \\
\hline & 0.0339 & 2.6260 & $2-15$ & 10 & 0.902 & 0.143 & $\log$ & 1.024 & comp & Subarctic Québec \\
\hline & 0.0963 & 2.4289 & $3-32$ & 734 & 0.960 & 12.66 & absw & $\mathrm{n} / \mathrm{a}$ & comp & Québec \\
\hline & 0.1137 & 2.3160 & $2-25$ & 68 & 0.983 & 0.148 & In & 1.011 & $\mathrm{n} / \mathrm{a}$ & Upper Great Lakes \\
\hline \multirow[t]{6}{*}{ SW: } & 0.0659 & 2.3570 & $2-30$ & 24 & 0.991 & 0.191 & $\ln$ & 1.018 & $\mathrm{n} / \mathrm{a}$ & Nova Scotia \\
\hline & 0.0888 & 2.3210 & $1-23$ & 24 & 0.980 & 4.555 & abs & $\mathrm{n} / \mathrm{a}$ & $8.2(50)$ & Minnesota \\
\hline & 0.0405 & 2.4743 & $2-34$ & 49 & 0.980 & 0.141 & In & 1.010 & $\mathrm{n} / \mathrm{a}$ & Nova Scotia \\
\hline & 0.0690 & 2.3387 & $0-37$ & 195 & 0.974 & 18.10 & abs & $\mathrm{n} / \mathrm{a}$ & comp & $\begin{array}{l}\text { New Brunswick, } \\
\text { Nova Scotia }\end{array}$ \\
\hline & 0.0177 & 2.8657 & $9-32$ & 734 & 0.900 & 14.51 & abs & $\mathrm{n} / \mathrm{a}$ & comp & Québec \\
\hline & 0.0525 & 2.3227 & $2-25$ & 68 & $\mathrm{n} / \mathrm{a}$ & $\mathrm{n} / \mathrm{a}$ & calc & $n / a$ & $\mathrm{n} / \mathrm{a}$ & Upper Great Lakes \\
\hline
\end{tabular}

Freedman et al., 1982 Grigal and Kernik, 1984a Ker, 1980 b

Ker, 1984

Moore and Verspoor, $1973^{j}$ Moore and Verspoor, $1973^{k}$ Ouellet, 1983

Perala and Alban, 1994

Freedman et al., 1982

Grigal and Kernik, 1984b

Ker, 1980 b

Ker, 1984

Ouellet, 1983

Perala and Alban. 1994 


\begin{tabular}{|c|c|c|c|c|c|c|c|c|c|c|}
\hline \multirow[t]{5}{*}{ SB: } & 0.0257 & 2.0555 & $2-30$ & 24 & 0.995 & 0.128 & $\ln$ & 1.008 & $\mathrm{n} / \mathrm{a}$ & Nova Scotia \\
\hline & 0.0438 & 1.8220 & $1-23$ & 24 & 0.930 & 0.914 & abs & $\mathrm{n} / \mathrm{a}$ & $8.2(50)$ & Minnesota \\
\hline & 0.0124 & 2.1815 & $2-34$ & 49 & 0.980 & 0.141 & $\ln$ & 1.020 & $n / a$ & Nova Scotia \\
\hline & 0.0035 & 2.7422 & $9-32$ & 734 & 0.860 & 2.150 & absw & $\mathrm{n} / \mathrm{a}$ & comp & Québec \\
\hline & 0.0186 & 2.0332 & $2-25$ & 68 & $\mathrm{II} / \mathrm{a}$ & $11 / \mathrm{a}$ & calc & $11 / \mathrm{a}$ & $11 / \mathrm{a}$ & Upper Great Lakes \\
\hline \multirow[t]{6}{*}{ ST: } & 0.0900 & 2,3026 & $2-30$ & 24 & 0.993 & 0.165 & $\ln$ & 1.014 & $n / a$ & Nova Scotia \\
\hline & 0.1183 & 2.2600 & $1-23$ & 24 & 0.980 & 4.288 & abs & $\mathrm{n} / \mathrm{a}$ & $8.2(50)$ & Minnesota \\
\hline & 0.0518 & 2.4321 & $2-34$ & 49 & 0.980 & 0.141 & $\ln$ & 1.010 & $\mathrm{n} / \mathrm{a}$ & Nova Scotia \\
\hline & 0.0849 & 2.3130 & $0-37$ & 195 & 0.976 & 19.30 & abs & $\mathrm{n} / \mathrm{a}$ & comp & $\begin{array}{l}\text { New Brunswick, } \\
\text { Nova Scotial }\end{array}$ \\
\hline & 0.0536 & 2.5656 & $3-32$ & 734 & 0.940 & 13.16 & absw & $n / a$ & comp & Québec \\
\hline & 0.2064 & 2.0370 & $0-16$ & 15 & 0.990 & 0.180 & $\ln$ & 1.016 & $\mathrm{n} / \mathrm{a}$ & Québec \\
\hline \multirow[t]{7}{*}{ FL: } & 0.0932 & 1.5518 & $2-30$ & 24 & 0.836 & 0.578 & In & 1.182 & $\mathrm{n} / \mathrm{a}$ & Nova Scotia \\
\hline & 0.0610 & 1.4110 & $1-23$ & 24 & 0.770 & 0.678 & $\mathrm{abs}$ & $n / a$ & $8.2(50)$ & Minnesota \\
\hline & 0.0790 & 1.7206 & $2-34$ & 49 & 0.910 & 0.312 & $\ln$ & 1.050 & $\mathrm{n} / \mathrm{a}$ & Nova Scotia \\
\hline & 0.0495 & 1.8761 & $0-37$ & 195 & 0.910 & 5.300 & abs & $\mathrm{n} / \mathrm{a}$ & comp & $\begin{array}{l}\text { New Brunswick, } \\
\text { Nova Scotia }\end{array}$ \\
\hline & 0.0179 & 2.3830 & $2-25$ & 68 & 0.928 & 0.373 & $\ln$ & 1.072 & $n / a$ & Upper Great Lakes \\
\hline & 0.2521 & 1.5940 & $0-16$ & 15 & 0.960 & 0.300 & $\ln$ & 1.046 & $\mathrm{n} / \mathrm{a}$ & Québec \\
\hline & 0.0004 & 3.2377 & $5-18$ & 20 & 0.865 & 0.172 & $\ln$ & 1.015 & $17(100)$ & Québec \\
\hline \multirow[t]{5}{*}{ BR: } & 0.0881 & 1.7374 & $2-30$ & 24 & 0.819 & 0.688 & $\ln$ & 1.267 & $\mathrm{n} / \mathrm{a}$ & Nova Scotia \\
\hline & 0.0251 & 2.0000 & $1-23$ & 24 & 0.910 & 0.977 & $a b s$ & $n / a$ & $8.2(50)$ & Minnesota \\
\hline & 0.0632 & 1.9421 & $2-34$ & 49 & 0.920 & 0.280 & $\ln$ & 1.040 & $\mathrm{n} / \mathrm{a}$ & Nova Scotia \\
\hline & 0.0287 & 2.2679 & 037 & 195 & 0.895 & 12.40 & $a b s$ & $\mathrm{n} / \mathrm{a}$ & comp & $\begin{array}{l}\text { New Brunswick. } \\
\text { Nova Scotia }\end{array}$ \\
\hline & 0.1529 & 1.9830 & $0-16$ & 15 & 0.990 & 0.230 & $\ln$ & 1.027 & $n / a$ & Québec \\
\hline
\end{tabular}

Spruce, Engelmann (Picea engelmannii Parry)

$\begin{array}{lllllllllll}\text { ST: } & 0.2844 & 1.3782 & 1-8 & 10 & 0.964 & 0.213 & \text { ln } & 1.023 & \text { comp } & \text { Idaho, Montana } \\ \text { FL: } & 0.3346 & 1.2765 & 1-23 & 13 & 0.917 & 0.430 & \text { ln } & 1.097 & \text { comp } & \text { Idaho, Montana } \\ \text { BR: } & 0.1687 & 1.5799 & 1-23 & 13 & 0.932 & 0.479 & \text { ln } & 1.122 & \text { comp } & \text { Idaho, Montana }\end{array}$

Spruce, Norway ( Picea abies (L.) Karst.)

$\begin{array}{lllllllllll}\text { AB: } & 0.2722 & 2.1040 & 12-44 & 30 & 0.960 & 0.152 & \ln & 1.012 & \mathrm{n} / \mathrm{a} & \text { New York } \\ \text { SW: } & 0.3832 & 1.8740 & 12-44 & 30 & 0.920 & 0.188 & \ln & 1.018 & \mathrm{n} / \mathrm{a} & \text { New York } \\ \text { SB: } & 0.0461 & 1.7800 & 12-44 & 30 & 0.910 & 0.194 & \ln & 1.019 & \mathrm{n} / \mathrm{a} & \text { New York } \\ \text { FL: } & 0.0031 & 2.8310 & 12-44 & 30 & 0.910 & 0.307 & \ln & 1.048 & \mathrm{n} / \mathrm{a} & \text { New York } \\ \text { BR: } & 0.0052 & 2.7320 & 12-44 & 30 & 0.900 & 0.308 & \ln & 1.049 & \mathrm{n} / \mathrm{a} & \text { New York }\end{array}$

Spruce, red (Picea rubens Sarg.)

\begin{tabular}{|c|c|c|c|c|c|c|c|c|c|c|}
\hline \multirow[t]{4}{*}{$\mathrm{AB}:$} & 0.1660 & 2.2417 & $1-31$ & 37 & 0.972 & 0.300 & $\ln$ & 1.046 & $\mathrm{n} / \mathrm{a}$ & Nova Scotia \\
\hline & 0.6149 & 1.5639 & $0-20$ & 30 & 0.881 & $\mathrm{n} / \mathrm{a}$ & $\log$ & $\mathrm{n} / \mathrm{a}$ & $n / a$ & New Brunswick \\
\hline & 0.1444 & 2.2604 & $0-37$ & 195 & 0.978 & 26.20 & abs & $\mathrm{n} / \mathrm{a}$ & comp & $\begin{array}{l}\text { New Brunswick, } \\
\text { Nova Scotia }\end{array}$ \\
\hline & 0.2066 & 2.1830 & $1-35^{a}$ & 15 & 0.982 & 0.107 & $\log$ & 1.013 & $\mathrm{n} / \mathrm{a}$ & New Hampshire \\
\hline \multirow[t]{3}{*}{ SW: } & 0.0779 & 2.3536 & $|-3|$ & 37 & 0.983 & 0.249 & $\ln$ & 1.031 & $n / a$ & Nova Scotia \\
\hline & 0.0690 & 2.3387 & $0-37$ & 195 & 0.974 & 18.10 & abs & $\mathrm{n} / \mathrm{a}$ & comp & $\begin{array}{l}\text { New Brunswick, } \\
\text { Nova Scotia }\end{array}$ \\
\hline & 0.0774 & 2.2380 & $1-35^{a}$ & 15 & 0.990 & 0.077 & $\log$ & 1.007 & $\mathrm{n} / \mathrm{a}$ & New Hampshire \\
\hline \multirow[t]{2}{*}{ SB: } & 0.0185 & 2.1879 & $|-3|$ & 37 & 0.975 & 0.280 & $\ln$ & 1.040 & $\mathrm{n} / \mathrm{a}$ & Nova Scotia \\
\hline & 0.0226 & 1.9961 & $1-35^{a}$ & 15 & 0.994 & 0.053 & $\log$ & 1.003 & $\mathrm{n} / \mathrm{a}$ & New Hampshire \\
\hline \multirow[t]{4}{*}{ ST: } & 0.0960 & 2.3288 & $1-31$ & 37 & 0.982 & 0.251 & $\ln$ & 1.032 & $\mathrm{n} / \mathrm{a}$ & Nova Scotia \\
\hline & 0.0849 & 2.3130 & $0-37$ & 195 & 0.976 & 19.30 & abs & $\mathrm{n} / \mathrm{a}$ & comp & $\begin{array}{l}\text { New Brunswick, } \\
\text { Nova Scotia }\end{array}$ \\
\hline & 0.1875 & 1.7743 & $0-20$ & 30 & 0.892 & $\mathrm{n} / \mathrm{a}$ & $\log$ & $n / a$ & $n / a$ & New Brunswick \\
\hline & 0.0979 & 2.2046 & $1-35^{a}$ & 15 & 0.994 & 0.070 & $\log$ & 1.006 & $\mathrm{n} / \mathrm{a}$ & New Hampshire \\
\hline FI: & 0.0150 & 2.2167 & $1-31$ & 37 & 0.884 & 0.637 & $\ln$ & 1.225 & $n / a$ & Nova Scotia \\
\hline
\end{tabular}

Freedman et al., 1982 Grigal and Kernik, 1984b Ker. 1980b Ouellet, 1983 Perala and Alban. 1994 Freedman et al., 1982 Grigal and Kernik. 1984 b Ker. 1980 b Ker, 1984

Ouellet, 1983

Rencz and Auclair, $1980^{\circ}$ Freedman et al.. 1982 Grigal and Kernik. 1984b Ker. 1980 b Ker, 1984

Perala and Alban, 1994 Rencz and Auclair, $1980^{\circ}$ Weetman and Harland. 1964 Freedman et al., 1982 Grigal and Kernik: 1984b Ker, 1980 b Ker. 1984

Rencz and Auclair. $1980^{\prime}$

Brown, $1978^{i}$ Brown, $1978^{\text {n }}$ Brown. $1978^{\text {t }}$

Jokela et al.. 1986 Jokela et al., 1986 Jokela et al., 1986 Jokela et al., 1986 Jokela et al.. 1986

Freedman et al., 1982 MacLean and Wein, 1976 Ker, 1984

Whittaker et al., 1974 Freedman et al., 1982 Ker. 1984

Whit aker et al, 1974 Freedman et al., 1982 Whittaker et al., 1974 Freedman et al., 1982 Ker, 1984

MacLean and Wein, 1976 Whittaker et al;; 1974 Freedman et al.. 1982 


\begin{tabular}{|c|c|c|c|c|c|c|c|c|c|c|c|}
\hline & 0.0495 & 1.8761 & $0-37$ & 195 & 0.910 & 5.300 & abs & $\mathrm{n} / \mathrm{a}$ & comp & $\begin{array}{l}\text { New Brunswick, } \\
\text { Nova Scotia }\end{array}$ & Ker, 198 \\
\hline \multirow[t]{3}{*}{ BR: } & 0.0293 & 2.0955 & $1-31$ & 37 & 0.871 & 0.640 & $\ln$ & 1.227 & $\mathrm{n} / \mathrm{a}$ & Nova Scotia & Freedman et al., 1982 \\
\hline & 0.0287 & 2.2679 & $0-37$ & 195 & 0.895 & 12.40 & abs & $\mathrm{n} / \mathrm{a}$ & comp & $\begin{array}{l}\text { New Brunswick, } \\
\text { Nova Scotia }\end{array}$ & Ker, 1984 \\
\hline & 0.0082 & 2.5428 & $1-35^{a}$ & 15 & 0.951 & 0.207 & $\log$ & 1.051 & $\mathrm{n} / \mathrm{a}$ & New Hampshire & Whittaker et al., 1974 \\
\hline
\end{tabular}

Spruce, Sitka ( Picea sitchensis (Bong.) Carr.)

$\begin{array}{lllllllllll}\text { ST: } & 0.0402 & 2.5520 & 3-78 & 21 & 0.970 & 0.050 & \text { ln } & 1.001 & \text { comp } & \text { Alaska } \\ \text { FL: } & 0.0030 & 2.7800 & 3-78 & 28 & 0.810 & 0.130 & \text { ln } & 1.008 & \text { comp } & \text { Alaska } \\ \text { BR: } & 0.0056 & 2.5180 & 3-78 & 28 & 0.800 & 0.130 & \text { ln } & 1.008 & \text { comp } & \text { Alaska }\end{array}$

Bormann, 1990

Bormann, 1990

Bormann, 1990

Spruce, white (Picea glauca (Moench) Voss)

\begin{tabular}{|c|c|c|c|c|c|c|c|c|c|c|c|}
\hline \multirow[t]{6}{*}{ AB: } & 0.0635 & 2.4800 & $3-25$ & 13 & 0.980 & $\mathrm{n} / \mathrm{a}$ & $\log$ & $\mathrm{n} / \mathrm{a}$ & $\mathrm{n} / \mathrm{a}$ & New Brunswick & Baskerville, 1965 \\
\hline & 0.1601 & 2.2413 & $2-30$ & 24 & 0.987 & 0.213 & $\ln$ & 1.023 & $\mathrm{n} / \mathrm{a}$ & Nova Scotia & Freedman et al., 1982 \\
\hline & 0.0777 & 2.4720 & $1-33$ & 115 & 0.980 & 12.70 & abs & $\mathrm{n} / \mathrm{a}$ & comp & Minnesota & Harding and Grigal, 1985 \\
\hline & 0.1037 & 2.2907 & $2-32$ & 44 & 0.990 & 0.141 & $\ln$ & 1.010 & $n / a$ & Nova Scotia & Ker, $1980 b$ \\
\hline & 0.1077 & 2.3308 & $0-39$ & 197 & 0.978 & 27.50 & abs & $\mathrm{n} / \mathrm{a}$ & comp & $\begin{array}{l}\text { New Brunswick, } \\
\text { Nova Scotia }\end{array}$ & Ker, 1984 \\
\hline & 0.1643 & 2.2480 & $2-25$ & 73 & 0.985 & 0.145 & $\ln$ & 1.011 & $\mathrm{n} / \mathrm{a}$ & Upper Great Lakes & Perala and Alban, 1994 \\
\hline \multirow[t]{6}{*}{ SW: } & 0.0537 & 2.3600 & $3-25$ & 13 & 0.980 & $\mathrm{n} / \mathrm{a}$ & $\log$ & $\mathrm{n} / \mathrm{a}$ & $\mathrm{n} / \mathrm{a}$ & New Brunswick & Baskerville, 1965 \\
\hline & 0.0731 & 2.3715 & $2-30$ & 24 & 0.990 & 0.201 & $\ln$ & 1.020 & $\mathrm{n} / \mathrm{a}$ & Nova Scotia & Freedman et al., 1982 \\
\hline & 0.0296 & 2.5930 & $1-33$ & 115 & 0.940 & 10.65 & abs & $\mathrm{n} / \mathrm{a}$ & comp & Minnesota & Harding and Grigal, 1985 \\
\hline & 0.0345 & 2.4847 & $2-32$ & 44 & 0.990 & 0.199 & $\ln$ & 1.020 & $\mathrm{n} / \mathrm{a}$ & Nova Scotia & Ker, $1980 \mathrm{~h}$ \\
\hline & 0.0376 & 2.4883 & $0-39$ & 197 & 0.974 & 17.90 & abs & $\mathrm{n} / \mathrm{a}$ & comp & $\begin{array}{l}\text { New Brunswick, } \\
\text { Nova Scotia }\end{array}$ & Ker, 1984 \\
\hline & 0.0698 & 2.3526 & $2-25$ & 72 & $\mathrm{n} / \mathrm{a}$ & $n / a$ & calc & $\mathrm{n} / \mathrm{a}$ & $\mathrm{n} / \mathrm{a}$ & Upper Great Lakes & Perala and Alban, 1994 \\
\hline \multirow[t]{5}{*}{ SB: } & 0.0031 & 2.6100 & $3-25$ & 14 & 0.980 & $\mathrm{n} / \mathrm{a}$ & $\log$ & $\mathrm{n} / \mathrm{a}$ & $n / a$ & New Brunswick & Baskerville, 1965 \\
\hline & 0.0246 & 2.0588 & $2-30$ & 24 & 0.987 & 0.198 & $\ln$ & 1.020 & $\mathrm{n} / \mathrm{a}$ & Nova Scotia & Freedman et al., 1982 \\
\hline & 0.0148 & 2.1390 & $1-33$ & 115 & 0.960 & 1.090 & abs & $\mathrm{n} / \mathrm{a}$ & comp & Minnesota & Harding and Grigal, 1985 \\
\hline & 0.0110 & 2.1547 & $2-32$ & 44 & 0.980 & 0.243 & $\ln$ & 1.030 & $\mathrm{n} / \mathrm{a}$ & Nova Scotia & Ker, $1980 \mathrm{~b}$ \\
\hline & 0.0200 & 1.9906 & $2-25$ & 73 & $\mathrm{n} / \mathrm{a}$ & $\mathrm{n} / \mathrm{a}$ & calc & $\mathrm{n} / \mathrm{a}$ & $\mathrm{n} / \mathrm{a}$ & Upper Great Lakes & Perala and Alban, 1994 \\
\hline \multirow[t]{4}{*}{ ST: } & 0.0957 & 2.3226 & $2-30$ & 24 & 0.989 & 0.201 & $\ln$ & 1.020 & $\mathrm{n} / \mathrm{a}$ & Nova Scotia & Freedman et al., 1982 \\
\hline & 0.0397 & 2.5360 & $1-33$ & 115 & 0.950 & 11.26 & abs & $\mathrm{n} / \mathrm{a}$ & comp & Minnesota & Harding and Grigal, 1985 \\
\hline & 0.0445 & 2.4370 & $2-32$ & 44 & 0.990 & 0.199 & $\ln$ & 1.020 & $\mathrm{n} / \mathrm{a}$ & Nova Scotia & Ker, $1980 \mathrm{~h}$ \\
\hline & 0.0445 & 2.4737 & $0-39$ & 197 & 0.974 & 20.10 & abs & $\mathrm{n} / \mathrm{a}$ & comp & $\begin{array}{l}\text { New Brunswick, } \\
\text { Nova Scotia }\end{array}$ & Ker, 1984 \\
\hline \multirow[t]{6}{*}{ FL: } & 0.0037 & 2.8500 & $3-25$ & 14 & 0.941 & $\mathrm{n} / \mathrm{a}$ & $\log$ & $\mathrm{n} / \mathrm{a}$ & $\mathrm{n} / \mathrm{a}$ & New Brunswick & Baskerville, 1965 \\
\hline & 0.0132 & 2.1816 & $2-30$ & 24 & 0.896 & 0.619 & $\ln$ & 1.211 & $\mathrm{n} / \mathrm{a}$ & Nova Scotia & Freedman et al., 1982 \\
\hline & 0.0162 & 2.2220 & $1-33$ & 115 & 0.780 & 3.600 & abs & $\mathrm{n} / \mathrm{a}$ & comp & Minnesota & Harding and Grigal, 1985 \\
\hline & 0.0369 & 1.9103 & $2-32$ & 44 & 0.930 & 0.312 & In & 1.050 & $\mathrm{n} / \mathrm{a}$ & Nova Scotia & Ker, $1980 \mathrm{~b}$ \\
\hline & 0.0610 & 1.8465 & $0-39$ & 197 & 0.900 & 6.800 & abs & $\mathrm{n} / \mathrm{a}$ & comp & $\begin{array}{l}\text { New Brunswick, } \\
\text { Nova Scotia }\end{array}$ & Ker, 1984 \\
\hline & 0.0165 & 2.3474 & $2-25$ & 73 & $\mathrm{n} / \mathrm{a}$ & $\mathrm{n} / \mathrm{a}$ & calc & $\mathrm{n} / \mathrm{a}$ & $\mathrm{n} / \mathrm{a}$ & Upper Great Lakes & Perala and Alban, 1994 \\
\hline \multirow[t]{5}{*}{ BR: } & 0.0047 & 2.7800 & $3-25$ & 14 & 0.941 & $\mathrm{n} / \mathrm{a}$ & $\log$ & $\mathrm{n} / \mathrm{a}$ & $n / a$ & New Brunswick & Baskerville, 1965 \\
\hline & 0.0166 & 2.1778 & $2-30$ & 24 & 0.874 & 0.688 & $\ln$ & 1.267 & $n / a$ & Nova Scotia & Freedman et al., 1982 \\
\hline & 0.0248 & 2.4300 & $1-33$ & 115 & 0.900 & 7.960 & abs & $\mathrm{n} / \mathrm{a}$ & comp & Minnesota & Harding and Grigal, 1985 \\
\hline & 0.0302 & 2.1368 & $2-32$ & 44 & 0.940 & 0.341 & $\ln$ & 1.060 & $\mathrm{n} / \mathrm{a}$ & Nova Scotia & Ker, $1980 b$ \\
\hline & 0.0435 & 2.1490 & $0-39$ & 197 & 0.873 & 15.30 & abs & $\mathrm{n} / \mathrm{a}$ & comp & $\begin{array}{l}\text { New Brunswick, } \\
\text { Nova Scotia }\end{array}$ & Ker, 1984 \\
\hline
\end{tabular}

Tanoak ( Lithocarpus densiflorus (Hook. and Arn.) Rehd.)

$\begin{array}{lllllll}\text { FL: } & 0.0261 & 1.8359 & 3-64 & 31 & 0.933 & 0.384\end{array}$

BR: $\quad 0.0223 \quad 2.3977 \quad 3-64$

In 1.077 comp Oregon, California

Snell and Little, $1983^{b}$

$\begin{array}{lll}31 & 0.938 & 0.481\end{array}$

In 1.123 comp

Oregon, California

Snell and Little, $1983^{\mathrm{b}}$

Willow (Salicaceae; willow family, includes poplars)
AB: $\begin{array}{llllll}0.0616 & 2.5094 & 4-20 & 180 \mathrm{n} / \mathrm{a} \quad \mathrm{n} / \mathrm{a}\end{array}$
calc $n / a$
$\mathrm{n} / \mathrm{a}$
Upper Great Lakes
Perala and Alban. 1994
$0.1619 \quad 2.0552 \quad 3-24^{\text {a }} \quad 30 \mathrm{n} / \mathrm{a} \quad \mathrm{n} / \mathrm{a}$
calc $\mathrm{n} / \mathrm{a}$
comp
Maine
Young et al., 1980 


\begin{tabular}{|c|c|c|c|c|c|c|c|c|c|c|c|}
\hline SW: & 0.0335 & 2.5259 & $4-20$ & 181 & $\mathrm{n} / \mathrm{a}$ & $\mathrm{n} / \mathrm{a}$ & calc & $\mathrm{n} / \mathrm{a}$ & $\mathrm{n} / \mathrm{a}$ & Upper Great Lakes & Perala and Alban. 1994 \\
\hline SB: & 0.0151 & 2.3323 & $4-20$ & 181 & $\mathrm{n} / \mathrm{a}$ & $n / a$ & calc & $n / a$ & $\mathrm{n} / \mathrm{a}$ & Upper Great Lakes & Perala and Alban. 1994 \\
\hline \multirow[t]{2}{*}{ ST: } & 0.0652 & 2.3391 & $3-8$ & 30 & 0.911 & $\mathrm{n} / \mathrm{a}$ & $\log$ & $n / a$ & comp & Maine & Ribe, 1973 \\
\hline & 0.0661 & 2.3321 & $3-24^{a}$ & 30 & 0.955 & $n / a$ & $\ln$ & $n / a$ & comp & Maine & Young at al., 1980 \\
\hline \multirow[t]{3}{*}{ FL: } & 0.0091 & 2.0645 & $4-20$ & 167 & $\mathrm{n} / \mathrm{a}$ & $\mathrm{n} / \mathrm{a}$ & cialc & $n / a$ & $n / a$ & Upper Great Lakes & Peralid and Albant 1994 \\
\hline & 0.0333 & 1.6442 & $3-8$ & 30 & 0.673 & $\mathrm{n} / \mathrm{a}$ & $\log$ & $\mathrm{n} / \mathrm{a}$ & comp & Maine & Ribe, 1973 \\
\hline & 0.0299 & 1.6921 & $3-24$ & 30 & 0.813 & $n / a$ & $\ln$ & $n / a$ & comp & Maine & Young et al., 1980 \\
\hline \multirow[t]{2}{*}{ BR: } & 0.0644 & 1.6624 & $3-8$ & 30 & 0.767 & $n / a$ & $\log$ & $n / a$ & comp & Maine & Ribe, 1973 \\
\hline & 0.0659 & 1.6514 & $3-24$ & 30 & 0.874 & $n / a$ & $\ln$ & $n / a$ & comp & Maine & Young et al., 1980 \\
\hline
\end{tabular}

\footnotetext{
DBH sample range uncertain and is estimated from either biomass tables or DBH distribution of the sampled stands.

${ }^{b}$ Equation was fitted by Ter-Mikaelian and Korzukhin using original data published in the reference.

' Canada-US included northern Wisconsin, New Hampshire, northcentral Minnesota, northwestern New Brunswick, Maine

${ }^{d}$ Equations for total aboveground biomass from Wiant et al. (1977) did not account for foliage weight.

Equation was developed for open-grown trees.

'Equation was developed for forest-growil tretes.

"Equation was developed for a "high forking class" that includes trees forking below the base of the live crown.

"Equation was developed for a "low forking class" that includes trees where the main stem extends into the live crown.

Baldwin (1989) fitted the equations in logarithmic form but calculated $R^{2}$ and SEE after backward conversion of the predictions to arithmetic units: these values were not comparable with those for other equations and, therefore, were not included in the table.

${ }^{i}$ Equation was developed for a spruce-lichen woodland.

${ }^{k}$ Equation was developed for a spruce-moss woodland.

${ }^{t}$ Diameter was measured at $1.2 \mathrm{~m}$ aboveground.
}

\section{References}

Agee, J.K., 1983. Fuel weights of understory-grown conifers in southern Oregon. Can. J. For. Res., 13: 648-656.

Baldwin, V.C.. Jr., 1989. Is sapwood area a better predictor of loblolly pine crown biomass than bole diameter? Biomass, 20: $177-185$

Baskerville, G.L., 1972. Use of logarithmic regression in the estimation of plant biomass. Can. J. For. Res., 2: 49-53.

Baskerville, G.L., 1965. Dry-matter production in immature balsam fir stands. Forest Science Monograph, Society of American Foresters, Washington, DC, $41 \mathrm{pp}$.

Bickelhaupt, D.H., Leaf, A.L. and Richards, N.A.. 1973. Effect of branching habit on aboveground dry weight estimates of Acer saccharum stands. In: H.E. Young (Editor), IUFRO Biomass Studies: Nancy, France, and Vancouver, B.C., Canada. University of Maine, College of Life Sciences and Agriculture, Orono, Maine, pp. 219-230.

Bormann, B.T., 1990. Diameter-based biomass regression models ignore large sapwood-related variation in Sitka spruce. Can. J. For. Res., 20: 1098-1104.

Brenneman, B.B., Frederick, D.J., Gardner, W.E., Schoenhofen, L.H. and Marsh, P.L., 1978. Biomass of species and stands of West Virginia hardwoods. In: P.E. Pope (Editor), Proceedings of Central Hardwood Forest Conference II. West LaFayette. Purdue University, pp. 159-178

Bridge, J.A., 1979. Fuelwood production of mixed hardwoods on mesic sites in Rhode Island. M.S. Thesis. University of Rhode Island, Kingston, Rhode Island, $72 \mathrm{pp}$.

Brown, J.K., 1978. Weight and density of crowns of Rocky Mountain conifers. U.S. For. Serv. Res. Pap. INT-197: 56

Campbell, J.S., Lieffers, V.J. and Pielou, E.C., 1985. Regression equations for estimating single tree biomass of trembling aspen: assessing their applicability to more than one population. For. Ecol. Manage., 11: 283-295.

Carpenter, E.M., 1983. Above-ground weights for tumarack in northeastern Minnesota. U.S. For. Serv. Res. Pap. NC. $245: 9$.

Cochran, P.H., Jennings, J.W. and Youngberg, C.T., 1984. Biomass estimators for thinned second-growth ponderosa pine trees. U.S. For. Serv. Res. Note PNW-415: 6.

Crow, T.R., 1971. Estimation of biomass in even-aged stand-regression and "mean tree" techniques. In: Forest Biomass Studies, 17th IUFRO Congress, Gainsville, Florida, 15-20 March 1971. pp. 35-50.

Crow, T.R. and Erdmann, G.G., 1983. Weight and volume equations and tables for red maple in the Lake States. U.S. For. Serv. Res. Pap. NC-242: 14

Crow, T.R. and Schlaegel, B.E.. 1988. A guide to using regression equations for estimating tree biomass. North. J. Appl. For. 5: $15-22$.

Feller, M.C., 1992. Generalized versus site-specific biomass regression equations for Pseudotsuga menziesti var. menżiesii and Thuja plicata in coastal British Columbia. Bioresour. Technol., 39: 9-16.

Freedman, B., Duinker, P.N., Barclay, H.. Morash, R. and Prager, U. 1982. Forest biomass and nutrient studies in central Nova Scotia. Can. For. Serv. Marit. For. Res. Cent. Inf. Rep., M-X-134: 126.

Gholz, H.L., Grier, C.C., Campbell, A.G. and Brown, A.T., 1979. Equations for estimating biomass and leaf area of plants in the pacific northwest. Oreg. State Univ. Sch. For. Res. Pap., 41: 39.

Grigal, D.F. and Kernik, L.K., 1984a. Generality of black spruce biomass estimation equations. Can. J. For. Res., 14: 468-470. 
Grigal, D.F. and Kernik, L.K., 1984b. Biomass estimation for black spruce (Picea mariana (Mill.) B.S.P.) trees. Minn. For. Res. Notes, 290: 4.

Harding, R.B. and Grigal, D.F., 1985. Individual tree biomass estimations for plantation-grown white spruce in northern Minnesota. Can. J. For. Res., 15: 738-739.

Hegyi, F., 1972. Dry matter distribution in jack pine stands in northern Ontario. For. Chron., 48: 193-197.

Hocker, H.W., Jr. and Earley, D.J., 1983. Biomass and leaf area cquations for northern forest species. N. H. $\Lambda$ gric. Exp. Stn. Univ. N. H. Res. Rep., 102: 27.

Honer, T.G., 1971. Weight relationships in open- and forest-grown balsam fir trees. In: Forest Biomass Studies. IUFRO Section 25. Yield and Growth, Working Group on Forest Biomass Studies, Life Sciences and Agriculture, Experimental Station, University of Maine at Orono, Miscellaneous Publication No. 132, pp. $65-78$.

Johnston, R.S. and Bartos, D.L., 1977. Summary of nutrient and biomass data from two aspen sites in western United States. U.S. For. Serv, Res. Note INT-227: 15.

Jokela, E.J., Van Gurp, K.P., Briggs, R.D. and White, E.H., 1986. Biomass estimation equations for Norway spruce in New York. Can. J. For. Res., 16: 413-415.

Ker, M.F., 1980a. Tree biomass equations for seven species in southwestern New Brunswick. Can. For. Serv. Marit. For. Res. Cent. Inf. Rep.. M-X-114: 18.

Ker, M.F., 1980b. Tree biomass equations for ten major species in Cumberland County, Nova Scotia. Can, For. Serv. Marit. For. Res. Cent. Inf. Rep., M-X-108: 26.

Ker, M.F., 1984. Biomass equations for seven major maritimes tree species. Can. For. Serv. Marit. For. Res. Cent. Inf. Rep. M-X-148: 54.

Kinerson, R.S. and Bartholomew, I., 1977. Biomass estimation equations and nutrient composition of white pine, white birch, red maple, and red oak in New Hampshire. N. H. Agric. Exp. Stn. Res. Rep., 62: 8.

King, W.W. and Schnell, R.L., 1972. Biomass estimates of black oak tree components. Tennessee Valley Authority, Division of Forestry, Fisheries, and Wildlife Development, Technical Note B 1 . Norris, Tennessee, $24 \mathrm{pp}$

Koerper. G.J. and Richardson, C.J., 1980. Biomass and net annual primary production regressions for Populus grandidentata on three sites in northern lower Michigan. Can. J. For. Res,, 10: $92-101$.

Krumlik, J.G., 1974. Biomass and nutrient distribution in two old growth forest ecosystems in south coastal British Columbia. M.Sc. Thesis, University of British Columbia, Vancouver, British Colunbia, $180 \mathrm{pp}$.

MacLean, D.A. and Wein, R.W., 1976. Biomass of jack pine and mixed hardwood stands in northeastern New Brunswick. Can. J. For. Res., 6: 441-447.

Marshall, P.L. and Wang, Y., 1995. Above ground tree biomass of interior uneven-aged Douglas-fir stands. Canada-British Columbia Partnership Agreement on Forest Resource Development: FRDA II. Working Paper WP-1.5-003. University of British Columbia, Vancouver, 23 pp.

Moore, T.R. and Verspoor, E., 1973. Aboveground biomass of black spruce stands in subarctic Quebec. Can. J. For. Res., 3: 596-598.

Ouellet, D., 1983. Biomass equations for black spruce in Quebec. Can. For. Serv. Inf. Rep. LAU-X-60(E): 27.

Pastor, J., Aber, J.D. and Melillo, J.M., 1984. Biomass prediction using generalized allometric regressions for some northeast tree species. For. Ecol. Manage., 7: 265-274.

Pastor, J. and Bockheim, J.G., 1981. Biomass and production of an aspen-mixed hardwood-spodosol ecosystem in northern Wisconsin. Can. J. For. Res., 11: 132-138.

Perala, D.A. and Alban, D.H., 1994. Allometric biomass estimators for aspen-dominated ecosystems in the Upper Great Lakes. U.S. For. Serv. Res. Pap. NC-134: 38.

Peterson, E.B., Chan, Y.B. and Cragg, J.B., 1970. Aboveground standing crop, leaf area, and caloric value in an aspen clone near Calgary, Alberta. Can. J. Bot., 48: 1459-1469.

Ralston, C.W., 1973. Annual productivity in a loblolly pine plantation. In: H.F. Young (Fditor), IUFRO Biomass Studies: Nancy, France, and Vancouver, B.C., Canada. University of Maine, College of Life Sciences and Agriculture, Orono, Maine, pp. 107-117.

Rencz, A.W. and Auclair, A.N., 1980. Dimension analysis of various components of black spruce in subarctic lichen woodland. Can. J. For. Res., 10: 491-497.

Reynolds, P.E., Carlson, K.G., Fromm, T.W., Gigliello, K.A. and Kaminski. R.J., 1978. Phytosociology, biomass, productivity, and nutrient budget for the tree stratum of a southern New Jersey hardwood swamp. In: P.E. Pope (Editor), Proceedings of Central Hardwood Forest Conference II. West LaFayette, Purdue University, pp. 123-139.

Ribe, J.H., 1973. Puckerbrush weight tables. Misc. Rep. 152. Life Sciences and Agricultural Experiment Station, University of Maine, Orono, MN, $92 \mathrm{pp}$.

Ruark, G.A., Martin, G.L. and Bockheim, J.G., 1987. Comparison of constant and variable allometric ratios for estimating Populus tremuloides Biomass. For. Sci., 33: 294-300.

Schmitt, M.D.C. and Grigal, D.F., 1981. Generalized biomass estimation equations for Betula papyrifera Marsh. Can. J. For. Res., 11: 837-840.

Snell, J.A. Kendall and Little, S.N., 1983. Predicting crown weight and bole volume of five western hardwoods. U.S. For. Serv. Gen. Tech. Rep. PNW-151: 37.

Stanek, W. and State, D., 1978. Equations predicting primary productivity (biomass) of trees, shrubs and lesser vegetation based on current literature. Can. For. Serv. Pac. For. Res. Cent. Inf. Rep., BC-X-183: 58.

Swank, W.T. and Schreuder, H.T., 1974. Comparison of three methods of estimating surface area and biomas for a forest of young eastern white pine. For. Sci., 20: 91-100.

Tritton, L.M. and Hornbeck, J.W., 1982. Biomass equations for major tree species of the Northeast. U.S. For. Serv. Gen. Tech. Rep. NE-69: 46.

Wang, J.R., Zhong, A.L., Simard, S.W. and Kimmins, J.P.. 1996. Aboveground biomass and nutrient accumulation in an age sequence of paper birch (Betula Papyrifera) in the Interior Hernlock zone, British Columbia. For. Ecol. Manage., 83: 27-38. 
Weetman, G.F. and Harland, R., 1964. Foliage and wood production in unthinned black spruce in northern Québec. For. Sci., 10: $80-88$.

Whittaker, R.H., Bormann, F.H., Likens, G.E. and Siccama, T.G., 1974. The Hubbard Brook ecosystem: Forest biomass and production. Ecol. Monogr., 44: 233-252.

Whittaker, R.H. and Woodwell, G.M., 1968. Dimension and production relations of trees and shrubs in the Brookhaven Forest, New York. J. Ecol., 56: 1-25.
Wiant, H.V., Jr., Sheetz, C.E., Colaninno, A., DeMoss, J.C. and Castaneda, F., 1977. Tables and procedures for estimatitiy weights of some Appalachian hardwoods. W. Va. Agric. Exp. Stn. Bull., 659(T): 36.

Young, H.E., Ribe, J.H. and Wainwright, K., 1980. Weight tables for tree and shrub species in Maine. Life Sciences and Agriculture Experiment Station. University of Maine at Orono, Miscellaneous Report 230. 84 pp. 